EUROPEAN ORGANIZATION FOR NUCLEAR RESEARCH

CERN-PPE/96-97

July 26,1996

\title{
The Forward Muon Detector of L3
}

\author{
The L3 F/B Muon Group
}

\begin{abstract}
The Forward-Backward muon detector of the L3 experiment is presented. Intended to be used for LEP 200 physics, it consists of 96 self-calibrating drift chambers of a new design enclosing the magnet pole pieces of the L3 solenoid. The pole pieces are toroidally magnetized to form two independent analyzing spectrometers. A novel trigger is provided by resistive plate counters attached to the drift chambers. Details about the design, construction and performance of the whole system are given together with results obtained during the 1995 running at LEP.
\end{abstract}

Submitted to Nuclear Instruments and Methods $A$ 


\section{The L3 F/B Muon Group}

A.Adam ${ }^{14}$ M.Aguilar-Benitez ${ }^{9}$ J.Alarcon, ${ }^{9}$ J.Alberdi, ${ }^{9}$ V.Alexandrov, ${ }^{13}$ A.Aloisio, ${ }^{10}$ M.G.Alviggi, ${ }^{10}$ H.Anderhub ${ }^{16}$ M.Ariza, T.Azemoon, T.Aziz, F.Bakker, S.Banerjee, K.Banicz, ${ }^{14}$ J.Barcala, U.Becker, J.Berdugo, P.Berges, B.L.Betev, ${ }^{16}$ A.Biland ${ }^{16}$ G.J.Bobbink, R.Bock, A.Böhm, ${ }^{1}$ V.Borissov, ${ }^{13}$ K.Bosseler ${ }^{1}$ Ph.Bouvier, E.Brambilla, ${ }^{10}$ J.D.Burger, C.Burgos, J.Buskens, J.C.Carlier, G.Carlino, ${ }^{10}$ J.Casaus, N.Cavallo, ${ }^{10}$ I.Cerjak, M.Cerrada, $^{9}$ Y.H.Chang, ${ }^{17}$ H.S.Chen, S.R.Chendvankar, ${ }^{4}$ V.Chvatchkine, M.Daniel, R.de Asmundis, ${ }^{10}$ G.Decreuse, ${ }^{6}$ K.Deiters ${ }^{15}$ L.Djambazov ${ }^{16}$ P.Duraffourg, F.C.Erné, ${ }^{2}$ H.Esser, S.Ezekiev ${ }^{13}$ G.Faber, ${ }^{16}$ M.Fabre ${ }^{15}$ G.Fernandez, K.Freudenreich, ${ }^{16}$ M.Fritschi, ${ }^{16}$ P.Garcia-Abia, A.Gonzalez, ${ }^{9}$ A.Gurtu, ${ }^{5}$ L.J.Gutay, ${ }^{14}$ Ch.Haller, W.D.Herold ${ }^{15}$ J.Herrmann, ${ }^{16}$ A.Hervé, H.Hofer ${ }^{16}$ H.Hofer ${ }^{6}$ M.Hofer ${ }^{16}$ T.Hofer, ${ }^{16}$ J.Homma, U.Horisberger, ${ }^{16}$ I.Horvath ${ }^{16}$ P.Ingenito, ${ }^{15}$ V.Innocente, ${ }^{10}$ I.Ioudine, M.Jaspers, ${ }^{2}$ P.de Jong, W.Kaestli, ${ }^{16}$ H.Kaspar, ${ }^{15}$ V.Kitov, ${ }^{13}$ A.C.König, ${ }^{11}$ V.Koutsenko, S.Lanzano, ${ }^{10}$ C.Lapoint, A.Lebedev, ${ }^{6}$ P.Lecomte ${ }^{16}{ }^{6}$ L.Lista, ${ }^{10}$ K.Lübelsmeyer, W.Lustermann, ${ }^{15}$ J.M.Ma, M.Milesi, A.Molinero, A.Montero, R.Moore, S.Nahn, J.Navarrete, M.Okle, ${ }^{9}{ }^{16}$ I.Orlinov ${ }^{9}{ }^{3}$ D.Ostojic, ${ }^{16}$ D.Pandoulas, ${ }^{1}$ P.Paolucci ${ }^{10}$ P.Parascandolo ${ }^{10}$ G.Passeggio ${ }^{10}$ S.Patricelli10 ${ }^{10}$ D.Peach, D.Piccolo, ${ }^{10}$ L.Pigni ${ }^{7}$ H.Postema, C.Puras, D.Ren, ${ }^{16}$ P.Rewiersma, A.Rietmeyer, K.Riles, J.Risco, ${ }^{2}$ A.Robohm, ${ }^{16}$ J.Rodin, $^{6}$ U.Roeser, ${ }^{16}$ L.Romero, W.van Rossum, ${ }^{2}$ H.Rykaczewski, ${ }^{16}$ M.E.Sarakinos, ${ }^{14}$ M.Sassowsky, V.Schegelsky ${ }^{12}$ N.Scholz ${ }^{16}$ K.Schultze, H.Schuylenburg, C.Sciacca, ${ }^{10}$ P.G.Seiler, ${ }^{15}$ T.Siedenburg, R.Siedling, B.Smith, V.Soulimov, ${ }^{10}$ K.Sudhakar, O.Syben, M.Tonutti, A.Udovcic, ${ }^{16}{ }^{1}$ J.Ulbricht, ${ }^{16}{ }_{\text {L.Veillet }}{ }^{7}$ M.Vergain, G.Viertel ${ }^{16}{ }^{6}$ H.P.von Gunten, ${ }^{16}$ An.A.Vorobyov, ${ }^{12}$ V.Vrankovic, ${ }^{15}$ A.de Waard, ${ }^{2}$ S.Waldmeier-Wicki, ${ }^{16}$ W.Wallraff ${ }^{1}$, H.C.Walter ${ }^{15}$ J.C.Wang, Z.L.Wei, R.Wetter, ${ }^{16}$ I.Weverling, C.Willmott, F.Wittgenstein, R.J.Wu, K.S.Yang, L.Zhou, Y.Zhou, H.L.Zuang,

1 I. Physikalisches Institut, RWTH, D-52056 Aachen, FRG

III. Physikalisches Institut, RWTH, D-52056 Aachen, FRG

2 National Institute for High Energy Physics, NIKHEF, and University of Amsterdam, NL-1009 DB Amsterdam, The Netherlands

3 University of Michigan, Ann Arbor, MI 48109, USA

4 Institute of High Energy Physics, IHEP, 100039 Beijing, China

5 Tata Institute of Fundamental Research, Bombay 400 005, India

6 Massachusetts Institute of Technology, Cambridge, MA 02139, USA

7 European Laboratory for Particle Physics, CERN, CH-1211 Geneva 23, Switzerland

8 University of Geneva, CH-1211 Geneva 4, Switzerland

9 Centro de Investigaciones Energeticas, Medioambientales y Tecnologicas, CIEMAT, E-28040 Madrid, Spain

10 INFN-Sezione di Napoli and University of Naples, I-80125 Naples, Italy

11 University of Nijmegen and NIKHEF, NL-6525 ED Nijmegen, The Netherlands

12 Nuclear Physics Institute, St. Petersburg, Russia

13 Bulgarian Academy of Sciences, Central Laboratory of Mechatronics and Instrumentation, BU-1113 Sofia, Bulgaria

14 Purdue University, West Lafayette, IN 47907, USA

15 Paul Scherrer Institut, PSI, CH-5232 Villigen, Switzerland

16 Eidgenössische Technische Hochschule, ETH Zürich, CH-8093 Zürich, Switzerland

17 High Energy Physics Group, Taiwan, China

$\S$ Corresponding author. e-mail freuden at afsmail.cern.ch 


\section{Contents}

1 Introduction $\quad 3$

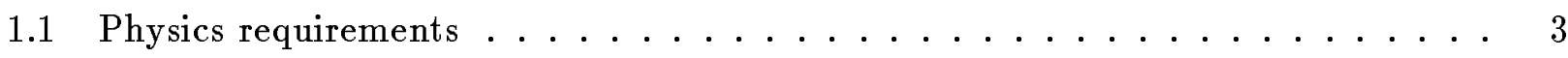

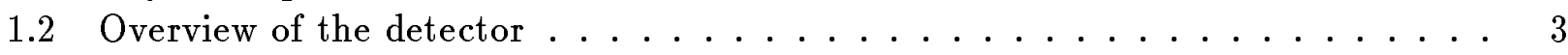

1.2.1 S-region : $44^{\circ} \geq \Theta \geq 36^{\circ} \ldots \ldots \ldots \ldots \ldots$

1.2.2 T-region : $36^{\circ} \geq \Theta \geq 24^{\circ} \ldots \ldots \ldots \ldots \ldots$

1.3 Detector design considerations . . . . . . . . . . . . . . . 5

2 Drift Chambers $\quad 6$

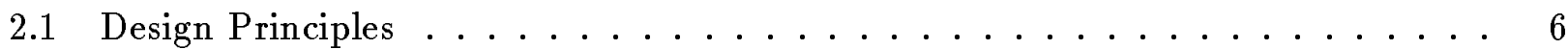

2.2 Calculation of Spatial Resolution ................. 7

3 Chamber construction and tests $\quad 7$

3.1 Chamber enclosures . . . . . . . . . . . . . . . . 7

3.2 Accurate wire positioning .................... 8

3.3 Chamber wiring procedures . . . . . . . . . . . . . 8

3.4 Chamber tests . . . . . . . . . . . . . . . . . . 9

4 Infrastructure and Electronics $\quad 9$

4.1 Front-end Boards and Amplifiers ................... 9

4.2 Discrimination Multiplexing and Readout . . . . . . . . . . . . . . 10

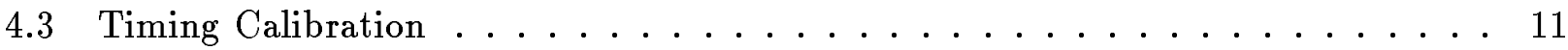

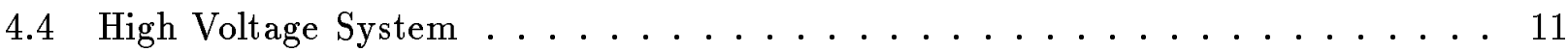

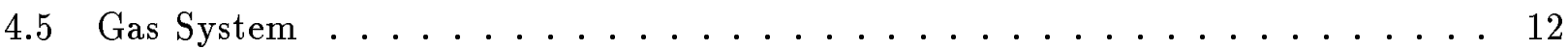

4.6 Temperature monitor system . . . . . . . . . . . . . . . . 12

5 Trigger RPCs $r$

5.1 The RPC System Layout . . . . . . . . . . . . . . . . . . . . . . . . . . . . . . . . . . .

5.2 Electronics and Tests . . . . . . . . . . . . . . . . 13

5.3 Level-1 Trigger Generation and Data Readout . . . . . . . . . . . . . . 14

6 F/B Toroids $\quad 15$

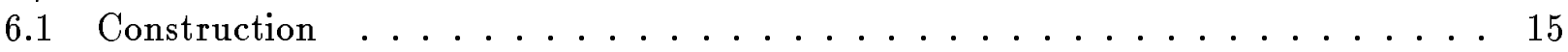

6.2 Magnetic field measurements . . . . . . . . . . . . . . . . . 15

6.3 Magnetic field calculation ..................... 16

7 System assembly and tests $\quad 16$

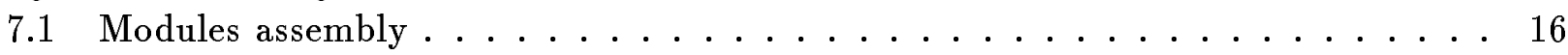

7.2 Tests and Spatial Resolution . . . . . . . . . . . . . . . . . . 16

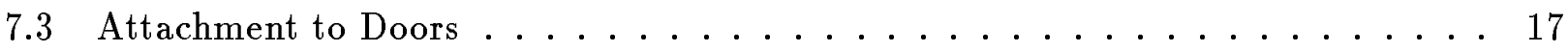

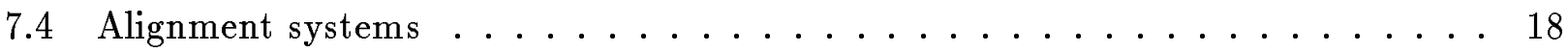

8 Performance at LEP $\quad 19$

8.1 Muon track reconstruction . . . . . . . . . . . . . . . 20

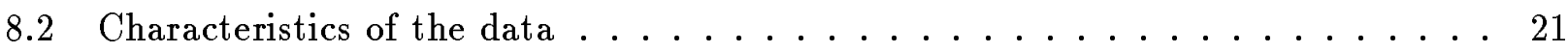

8.3 Spatial Resolution . . . . . . . . . . . . . . . . . . 21

8.4 Momentum Resolution ...................... 22

8.5 RPC Performance....................... 23 


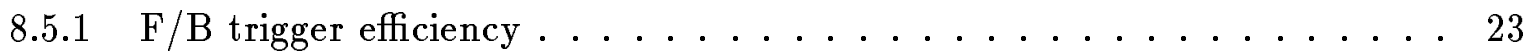

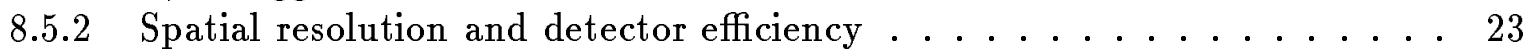

8.5.3 Time resolution and bunchlet identification . . . . . . . . . . . . . 24

\section{Introduction}

\subsection{Physics requirements}

The higher energies of LEP 200 require a more complete coverage for muon detection since the existing L3 barrel detector covers (with full resolution) only polar angles between $44^{\circ}$ and $136^{\circ}$. The new forward-backward (F/B) detector adds muon acceptance in the polar angle range (with respect to the beam axis) $44^{\circ} \geq \Theta \geq 24^{\circ}$ (see Fig. 1). The measured solid angle coverage is increased to $92 \%$ of $4 \pi$. This is essential for the process $\mathrm{e}^{+} \mathrm{e}^{-} \rightarrow \mathrm{W}^{+} \mathrm{W}^{-} \rightarrow \mu \nu_{\mu}+$ hadrons which provides a critical test to the so far successful Standard Model [1]. A simulated event of $\mathrm{e}^{+} \mathrm{e}^{-} \rightarrow \mathrm{W}^{+} \mathrm{W}^{-} \rightarrow \mu \nu_{\mu}+$ two jets is shown in Fig. 2. The manifestations of gauge cancellations are most sensitive in the $\mathrm{F} / \mathrm{B}$ region which is covered by the new detector. In addition, the acceptance for the Higgs particle search in the $60-95 \mathrm{GeV}$ mass range is doubled, and the acceptance for muons originating from possible new particles is substantially increased. Two photon physics also benefits from this detector since abundant events at these energies characteristically contain particles at low angles. Therefore, the F/B detector represents an important extension of the existing barrel muon detector [2].

\subsection{Overview of the detector}

The F/B detector consists of 96 precise drift chambers mounted in three layers (FI, FM, FO) on either side of the two magnet pole pieces (called doors in the following) which are toroidally magnetized by newly added coils as indicated in Fig. 1 . The 96 chambers have a trapezoidal shape (see Fig. 3). For triggering, the middle (FM) and outer (FO) layers are covered by Resistive Plate Counters (RPC), 192 units in total. Detector features are summarized in Table 1. 


\begin{tabular}{|l|l|}
\hline Number of chambers & 96 \\
Number of modules & $2 \times 16$ \\
Signal wires & $96 \times 256$ \\
Wire length & $0.54-2.84 \mathrm{~m}$ \\
Track segments in $x(\Phi)$ & $\mathrm{N}=2 \times 4$ wires \\
Track segments in $y(r)$ & $\mathrm{N}=1 \times 4$ wires \\
Coordinate accuracy & $250 \mu \mathrm{m} / \sqrt{\mathrm{N}}$ \\
Drift chamber gas & $\mathrm{Ar}: \mathrm{CO}_{2}: \mathrm{iC}_{4} \mathrm{H}_{10}=86: 10: 4 \%$ \\
Gas amplification & $5 \times 10^{4}$ \\
Preamplifier & $120 \mathrm{mV} / \mu \mathrm{A}$ \\
Threshold & $40 \mathrm{mV}$ \\
RPC counters & 192 \\
RPC gas & $\mathrm{Ar}: \mathrm{C}_{4} \mathrm{H}_{10}: \mathrm{CBrF}_{3}=58: 38: 4 \%$ \\
Timing accuracy & $\leq 3 \mathrm{~ns}$ \\
Magnetic field: solenoid & $0.51 \mathrm{~T}$ average \\
Magnetic field: toroid & $1.24 \mathrm{~T}$ average \\
Alignment absolute $/$ relative & $O(100 \mu \mathrm{m} / 50 \mu \mathrm{m})$ \\
$\Delta p / p(p=45 \mathrm{GeV}): 44^{\circ} \geq \Theta \geq 36^{\circ}$ & $4-23 \%$ \\
$\Delta p / p: 36^{\circ} \geq \Theta \geq 24^{\circ}$ & $30 \%$ \\
\hline
\end{tabular}

Table 1: Design parameters of the L3 forward backward muon system

As indicated in Fig. 1, there are two complementary regions, S and T. In each region a different method to measure the muon momentum is used.

\subsubsection{S-region : $44^{\circ} \geq \Theta \geq 36^{\circ}$}

Here muons are analyzed by measuring the helical bend in the $0.51 \mathrm{~T}$ solenoid field with the central drift chambers MI, MM and the new forward chamber FI. A spatial accuracy of $90 \mu \mathrm{m}$ given by the FI chamber, together with an ideal alignment comparable to the one reached in the central detector of less than $30 \mu \mathrm{m}$, would provide a muon momentum resolution varying from 3 to $19 \%$ for $p=45 \mathrm{GeV}$. This is not possible in practice, since the magnet doors move with respect to the central detector. The positioning of the 400 ton doors is reproducible to a few $\mathrm{mm}$, and deflections under magnetic field are in the range 1 to $8 \mathrm{~mm}$. The resolution shown in Fig. 4, varying from 4 to $23 \%$, assumes that a monitor system using sophisticated sensors provides corrections accurate to the order of $100 \mu \mathrm{m}$.

\subsubsection{T-region : $36^{\circ} \geq \Theta \geq 24^{\circ}$}

In this region, the deflection by the $1.24 \mathrm{~T}$ toroidal field in the $0.9 \mathrm{~m}$ iron doors is used to measure muon momenta. The chambers FI, FM and FO form independent spectrometers with excellent relative alignment. The resolution, also shown in Fig. 4 , is limited to $30 \%$ by multiple scattering. Since it is independent of momentum, the muon charge can be determined up to the highest LEP200 momenta. 


\subsection{Detector design considerations}

The S-region requires FI chambers of high precision in order to measure the muon track sagitta of about $3800 \mu \mathrm{m}$ at $45 \mathrm{GeV}$ to $90 \mu \mathrm{m}$ accuracy. In addition, the left-right ambiguity present in drift chambers must be resolvable in the track reconstruction. The design had to satisfy a serious constraint on the chamber thickness which is imposed by the $20 \mathrm{~cm}$ clearance left when the 400-ton doors are shut. Several chamber types were considered: (a) Jet chambers similar to the ones used in the barrel [2] could not be used due to space limitations in the FI volume. (b) Arrays of $1 \times 1 \mathrm{~cm}^{2}$ drift tubes required too many electronic channels and do not reach accuracies comparable to the barrel chambers. (c) Honeycomb chambers [3] with cathode strip readout could reach the accuracy, but were under development and had not been built in the large sizes required. (d) Therefore, a new type of chamber using a robust, self supporting I-beam structure with drift cells was designed [4]. The drift field homogeneity was improved by placing guard strips above and below the wire plane.

The layout of such a chamber is schematically shown in Fig. 5. A single chamber measures the muon track in two projections independently. The $y-z$ projection ${ }^{1)}$ of the track is measured in one layer (Y) of drift cells with 4 wires each. Each such layer provides a track segment accurate enough to check the pointing to the $e^{+} e^{-}$interaction point for straightforward reconstruction. The $x-z$ projection is measured in two layers (X and $\mathrm{W}$ ) of drift cells with 4 wires each; by staggering these layers by half a cell width, the left-right ambiguity in this projection is easily resolved. This projection is crucial for momentum measurements in the S-region, and one expects a spatial accuracy [5] of

$$
250 \mu \mathrm{m} / \sqrt{8 \text { wires }}=90 \mu \mathrm{m}
$$

which is adequate. The linkage of the segments from the $\mathrm{X}$ and $\mathrm{W}$ layers yields a calibration of the drift velocity without laser- or other calibrations. Once the velocity is known, the matching between these segments identifies the LEP machine bunch uniquely, as wrong bunch hypotheses would lead to quantized offsets between the segments.

The trigger in the T-region is provided by a new technology using RPCs, which can handle LEP rates well. Scintillation counters close to the interaction point which fully cover the forward region are also used.

The large F/B detector had to be constructed quickly with limited resources. To achieve this, the following considerations were crucial:

- The realization that the $0.9 \mathrm{~m}$ thick magnet door was not saturated by the solenoid return field in the T-region. Overlaying a toroidal field of $1.24 \mathrm{~T}$ required coils with $6.3 \mathrm{kA}$ in 36 turns per side.

- Modularity: All chambers were chosen of the same size and designed for mass production in semi-automatic assembly lines at different institutes.

- Multiplexing: The number of time digitizers (TDCs) was reduced by a factor of 8 .

- Preassembly: Drift chambers and RPCs were assembled into aligned detector modules for fast installation in the experiment. The modules are self-triggering, and hence are tested independently.

\footnotetext{
1) Octant by octant, we use a local coordinate system in which $z$ coincides with the electron beam direction in LEP, $y$ points radially outward along the octant centerline, and $x$ is perpendicular to $y$ and $z$. This corresponds approximately (along the octant centerline exactly) to $y=r$ and $x=\Phi$. The solenoidal magnetic field in L3 bends the muon in the $r-\Phi$ plane, the toroidal field bends in the $r-z$ plane.
} 


\begin{tabular}{|l|l|}
\hline 4 signal wires $30 \mu \mathrm{m} \mathrm{W}-\mathrm{Rh}$ & $130 \mathrm{~g}$ tension \\
5 field wires $75 \mu \mathrm{m} \mathrm{Cu}-\mathrm{Be}$ & $385 \mathrm{~g}$ tension \\
Drift distance & $51.5 \mathrm{~mm}$ \\
Cell height & $60 \mathrm{~mm}$ \\
Sense Wire spacing & $9 \mathrm{~mm}$ \\
Sense wire voltage & $+3800 \mathrm{~V}$ \\
Field wire voltage & $+2840 \mathrm{~V}$ \\
Guard strip voltage & $+2870 \mathrm{~V}$ \\
I-beam voltage & $-3000 \mathrm{~V}$ \\
Drift field & $1.1 \mathrm{kV} / \mathrm{cm}$ \\
\hline
\end{tabular}

Table 2: Cell parameters

\section{Drift Chambers}

\subsection{Design Principles}

All chambers consist of three layers (Y,X,W) composed of the new drift cell design [4] (Fig. 5). In the S-region the bending coordinate is measured by the $\mathrm{X}$ and $\mathrm{W}$ layers containing $19(18)$ cells each, with the orthogonal coordinate measured by the Y layer containing 27 cells. In the T-region, the main role in measuring the polar angle deflection of focussed or defocussed tracks is played by the $\mathrm{Y}$ layers.

The four-wire cells provide convenient track segments for reconstruction. Their parameters are given in Table 2. One layer is sufficient for the $y$ coordinate, since the $\Theta$ angle from the interaction point must be compatible with the four-wire local slope, hence resolving the leftright ambiguity. In the $x$ coordinate, the ambiguity is eliminated by having two offset layers. The chambers are self-calibrating for $90^{\circ}$ incident tracks (see Fig. 5) by the condition: $t_{1}+t_{2}=$ $52.5 \mathrm{~mm} / v_{e f f}, v_{\text {eff }}$ being the effective drift velocity. The chambers are "time stamping" since the quantized displacement between the two track segments in $\mathrm{X}$ and $\mathrm{W}$ is $\Delta x=2 v_{\text {eff }} \times \Delta t$, where $\Delta t$ is the time to the next (previous) accelerator bunch.

The electrostatic configuration of the drift cells is given in Fig. 6 and Table 2. The chamber cover is at ground potential. A guard strip above and below the wire plane shapes the field lines better and ensures uniform gain on the four wires.

All chambers have a standard size with three layers of identical $105 \mathrm{~mm}$ wide drift cells. They have been economically produced by gluing three layers of insulated I-beams on $2 \mathrm{~mm}$ aluminium sheets. This results in a structurally rigid body of $18.2 \mathrm{~cm}$ thickness only. A trapezoidal frame encloses the gas volume and contains the electronics on the outside periphery. Despite space limitations, the chamber FI reaches a spatial accuracy comparable to that of the central detector [6].

New safety regulations did not permit using the Argon-Ethane gas mixture used in the central detector. In a special effort [7], a non flammable gas similar in drift velocity was determined : Ar: $\mathrm{CO}_{2}: \mathrm{iC}_{4} \mathrm{H}_{10}=86: 10: 4$ (see Fig. 7a). It has a Lorentz deflection of only $15^{\circ}$ at $0.5 \mathrm{~T}$ (see Fig $7 \mathrm{~b}$ ). Operation inside and outside the L3 magnet in a velocity plateau is possible at about $1.1 \mathrm{kV} / \mathrm{cm}$. Corrections [8] are however needed due to the unavoidable nitrogen contamination (about $0.5 \%$ ) in a large system. In addition, this mixture allows to calculate and correct [9] for the effects from inhomogeneity and direction changes of the magnetic field, which occur at the edges of the doors. 
To obtain mechanical accuracies better than the spatial resolution of $90 \mu \mathrm{m}$, the 4 wires of each cell were pulled over a precisely ground glass cylinder which in turn was glued to plastic inserts in the chamber endframes with an accuracy better than $50 \mu \mathrm{m}$ as will be described in section 3.2.

\subsection{Calculation of Spatial Resolution}

The optimization of the voltages was done with the program WIRCHA [10]. By adjusting the mechanical width and potential of the guard strip, the electrostatic deflection of the outer signal wires was compensated, and the electrical field on the surface was made close to the one on the inner wires. The sharing of potentials between I-beam and wire plane was chosen to make the arrival time of drifting electrons among the four wires as similar as possible. Including transversal and longitudinal diffusion, we have calculated the propagation times for electrons drifting from a minimum ionizing track of 80 electrons $/ \mathrm{cm}$ with 18 primary ionizations $/ \mathrm{cm}$ (Fig. 8a).

In figure $8 \mathrm{~b}$ the jitter in the arrival time of the $8^{\text {th }}$ electron from a track $26 \mathrm{~mm}$ away is given for the inner and outer wires. Neglecting fluctuations in gas amplification this corresponds to signal/threshold ratio of $\approx 10$, which is used in operation. The single wire resolution for various drift distances was then estimated by taking the arrival time of the $8^{\text {th }}$ electron as the time of reaching the discriminator threshold. Taking $v=5.1 \mathrm{~cm} / \mu \mathrm{s}$, the jitter for each drift distance is converted into a spatial resolution and plotted in Fig. 8c. The predicted average single wire resolution is of the order of $200 \mu \mathrm{m}$, although the exact value depends on the detailed cluster structure of the ionization, and can easily reach values of $250 \mu \mathrm{m}$. Including a known magnetic field perpendicular to E essentially changes the time - distance relation but a similar resolution is obtained.

\section{Chamber construction and tests}

\subsection{Chamber enclosures}

The mass production of chambers utilized pre-fabricated aluminium I-profiles, which were glass fiber insulated and $2 \mathrm{~mm}$ aluminium sheets already equipped with electrode strips. They were assembled to form the three layer body (see Fig. 9) in 6 gluing steps. Assembly tables flat to $50 \mu \mathrm{m}$ held the aluminium sheets while special 0.5 ton pneumatic templates positioned the I-profiles, ensuring correct relative spacing. Vertical alignment bolts attached to the tables provided precise absolute alignment of the I-profiles and the aluminium sheet, and maintained the alignment during the bonding time of the glue.

The chamber body was framed by $3 \mathrm{U}$-shaped extruded aluminium profiles and 1 aluminium plate, the centerline frame, $20 \mathrm{~mm}$ thick and $3 \mathrm{~m}$ long. The U-profiles house all amplifiers, multiplexers, signal and high voltage cables, as well as gas inlet and outlet. Corner blocks allow to join these profiles to a frame and are also used to locate the chamber fixation feet which are used to mount the chambers on the magnet doors.

The chamber mechanical enclosure was completed when gluing the last cover sheet and the four aluminium endframes. During serial production, a rate of 2.5 chamber bodies per week was achieved. 


\subsection{Accurate wire positioning}

Before gluing to the chamber body, each endframe had been carefully prepared for the subsequent positioning of the wires. To this purpose plastic inserts were glued into holes machined in the frames. The edge of a glass cylinder, incorporated in each such insert (see Fig. 10), served to define the position of the wires for every chamber cell. It was thus essential to ensure the correct glass cylinder positioning during the process of gluing the plastic inserts into the endframes. This was achieved by two specially prepared templates [11].

As sketched in Fig. 11a each glass cylinder is positioned by two pins. On each template, pin locations were checked by using a laser interferometer system and found to be accurate to $10 \mu \mathrm{m}$ when compared to nominal values (see Fig. 11b). Aluminium was also chosen as material for the template ensuring that temperature variations would not affect the precision in the relative positioning of the glass cylinders.

A possible distortion ("waviness") of the $3.4 \mathrm{~m}$ long U profile mounted on the slanted $\left(22.5^{\circ}\right)$ chamber side would directly affect the relative positioning of the $\mathrm{X}$ and $\mathrm{W}$ wire planes (and to a lesser extent also that of the $\mathrm{Y}$ wires) in contrast to the other profiles. Therefore, special care was taken to keep this profile flat while gluing the glass cylinders into place. To this purpose, the template-profile combination was placed on an extremely stable horizontal granite block and was aligned with respect to this block during the gluing process. Furthermore, precautions were taken to maintain this flatness while turning the profile to mount it onto the chamber body.

After gluing, the positions of the glass cylinders with respect to the template pins were checked, by demanding that a $10 \mu \mathrm{m}$ thick metal foil should not fit between the cylinder and the pins. Moreover, for a sample of the first frames prepared, cylinder positions were also measured by laser interferometry and found to be correct to $15 \mu \mathrm{m}$.

During the gluing of the prepared endframes to the chamber body, proper mechanical alignment of frames relative to each other was achieved by interconnecting them with precisely machined devices, also made of aluminium to provide automatic compensation for temperature variations.

All these procedures ensured that the accuracy obtained in the relative distances between any of the wire planes inside a chamber is better than $50 \mu \mathrm{m}$. This is essential from the point of view of alignment with respect to the rest of the detector. Each chamber can be considered as a unit with an internal geometry with well defined locations of all wires.

\subsection{Chamber wiring procedures}

After mechanical assembly and several additional operations, the sense and field wires were inserted into the completed chamber bodies. The wires were crimped pneumatically on slotted cubes of a $\mathrm{Cu}$-Te alloy inserted into the holes of a polycarbonate block. Fig. 12 shows the different $\mathrm{Cu}$-Te cubes that were used for the fixation and electrical connection of the signal and field wires. The length of the sides of all cubes is $4 \mathrm{~mm}$. To achieve precise positioning, injection molded polycarbonate pieces containing holes were developed, in which the $\mathrm{Cu}-\mathrm{Te}$ cubes were glued (also shown in Fig. 12). This guaranteed electrical isolation up to $5 \mathrm{kV}$, as well as quick fixation of a whole group of nine signal and field wires. The $\mathrm{Cu}$-Te cubes for the two chamber sides where the electronics are mounted ("active" sides) are produced with cylindrical pins (see Fig. 12). After goldplating of the cubes, the pins serve to directly plug-in the preamplifier boards, as well as to connect the high voltage distribution system. Further details on the wire fixation scheme are given in Ref. [12]. 
To allow fast and efficient wire stringing, a computer controlled wiring machine which simultaneously prepared a plane of four signal and five field wires was built (Fig. 12). A polycarbonate block with the wires crimped on was moved by a computer controlled step motor until the wires reached the appropriate length. Before reaching the final length, magnetic brakes blocked the wire spool and tension was applied to the wires via a series of guide rollers and weights. Then the block was slowly moved to the final length, and the other ends of the wires were crimped to the $\mathrm{Cu}$-Te cubes of two polycarbonate blocks, one for the end of the current wire plane and the other for the start of the next. The wires were cut between these two blocks and the prepared plane was transferred into the chamber.

When installed in the chamber, the wires were again stretched to the same length as on the wiring machine, namely to the length of the drift cell, and therefore have the design tension values. The tension of each wire was checked by measuring its oscillation frequency in an external magnetic field. Typical distributions of the tension measured for field and signal wires are shown in Fig. 13(a,b), where the deviations from the intended values of $3.78 \mathrm{~N}$ and 1.28 $\mathrm{N}$, respectively, are plotted. Most of the measurements lie within limits of $\pm 4 \%$. In particular, negative deviations are less than $4 \%$ for all long wires, thus ensuring a gravitational sag of less than $100 \mu \mathrm{m}$.

\subsection{Chamber tests}

All chambers went through integrity checks at several stages before final installation. Gas tightness was checked by measuring the pressure drop vs. time of each chamber pressurized with $10 \mathrm{~cm}$ of water column above atmospheric pressure. Chambers were accepted, if the decay constant was larger than 120 minutes. Typical values were 360 minutes. Leaks, usually due to displaced cell covers or bad gas fittings, were localized with a hand held gas sniffer and repaired. High voltage problems were checked simply by monitoring currents while slowly raising the high voltage. Any problems were usually solved by inspection. Overall performance, including cabling, connections, electronics, and high voltage problems undetectable by current monitoring, was checked by measuring the cosmic rate (see Fig. 14 for an example of one chamber). The histograms usually pointed to the problems.

\section{Infrastructure and Electronics}

\subsection{Front-end Boards and Amplifiers}

The whole forward muon chamber system has 24576 signal wires for drift time measurements (256/chamber). A double layer front-end board provides the high voltage supplies via $1 M \Omega$ resistors, carries a four fold hybrid preamplifier circuit and distributes the time calibration signal TOCAL to the preamplifier inputs.

This board is connected to the drift cell via an additional board, which also secures gas tightness. The preamplifiers are coupled to the anodes by a capacitor of $100 \mathrm{pF}$ and a serial protection resistor of $150 \Omega$. All input and output cables have connectors to allow an easy assembly and exchange of boards if necessary. They are mounted with three screws to the solid chamber frame which gives an electrical reference ground.

The preamplifier is based on the design of the "L3 wire amplifier" [14], modified for higher gain and a layout as a four-fold hybrid to match the cells. The basic amplification structure consists of two "feedback pairs" $[15,16]$ in cascade, which is a simple solution for a broad band, 
high gain, low power amplifier with feedback stabilized current gain (see Fig. 15). The step response was measured to be $2 \times 60 \mathrm{mV} / \mu \mathrm{A}$. The transistors are npn type BFS17. Higher bandwidth types in the first stage result in a slightly better transfer performance, but also in an increased probability for damages caused by the discharge of the coupling capacitor in case of sparks. The quasi complementary outputs are ac-coupled and matched to twisted pair cables. The equivalent noise charge (ENC) was measured without capacitive load at the input to be less than $2000 \mathrm{e}$, whereas the slope of the noise as a function of the detector capacitance is about $40 \mathrm{e} / \mathrm{pF}$.

The charge gain and the rise time of the pulse response were checked for all preamplifier channels. Simulating the chamber capacitance by a $30 \mathrm{pF}$ capacitor, the charge gain was determined to be $2 \times 6.7 \pm 0.5 \mathrm{mV} / \mathrm{fC}$ and the rise time under the same conditions was found to be $6.7 \pm 0.3 \mathrm{~ns}$. From the numbers obtained for the gain, the ENC at $30 \mathrm{pF}$ and the threshold of the discriminators of $40 \mathrm{mV}$, the value of the effective threshold at the input was determined to be $6 \times \mathrm{ENC}$ or 20000 e.

The time calibration signal, individually distributed to all front-end boards, is a $-20 \mathrm{mV}$ step signal and delivers via the $2 \mathrm{pF}$ coupling capacitor a $-40 \mathrm{fC}$ input charge and a $550 \mathrm{mV}$ output pulse.

\subsection{Discrimination Multiplexing and Readout}

The sense wire signals, after amplification and discrimination, are multiplexed in a 8 to 1 scheme to reduce the amount of TDC channels to 3072 . For read out, the 96 channel TDCs $\left.{ }^{2}\right)$ are organized in a Forward and a Backward part of 16 modules each in one FastBus crate.

Each amplified sense wire signal is terminated into a high speed discriminator, which has differential $50 \Omega$ inputs, a minimum threshold of $5 \mathrm{mV}$, a built-in hysteresis of $3 \mathrm{mV}$ and a monoflop action of $100 \mathrm{~ns}$. The discriminator design is centered around a high speed comparator ${ }^{3)}$. Propagation delay dispersion is almost fully determined by the rise time of the wire amplifier. The output signals are FAST TTL with a rise time of $3 \mathrm{~ns}$. In L3 the threshold is set to 40 $\mathrm{mV}$ for average pulses of $350 \mathrm{mV}$.

The circuit combines 8 discriminator signals into one output (see Fig. 16). The signals come from different, non-adjacent cells [17], so that usually there is at most one signal active. When multiplexing the drift times, the channel address is kept in local FIFO registers. These addresses, in combination with the TDC timing data, are used to reconstruct muon tracks. An overview of the read out electronic set-up can be seen in Fig. 17.

Within a chamber, there are 8 discriminator multiplexer cards (DIMUX) with 32 channels each read out by a single detector communication card (DETCOM) which sits in the top corner of the chamber as shown in Fig. 17. The DIMUX cards are enabled during the drift time interval of $1.2 \mu \mathrm{s}$ after the beam crossing. During this time all read out electronic circuits are in the stand-by mode, only the discriminators and hit registers are active. No clocks are running, nor data being transferred, that may cause electronic noise in the front ends. Outside the drift gate the inputs are not sensitive.

The DETCOM also distributes the fast Gate and Clear signals to the DIMUX cards together with the threshold voltage for the discriminators. In a halfwheel, the 8 DETCOM cards are sequentially addressed by the controller through the "Octopus Box" to the "FBROC" cards which allow to identify wires numbers with hits as shown in Fig. 17.

\footnotetext{
2)LECROY TDC LRS 1879

3) AD9696KR
} 


\subsection{Timing Calibration}

Relative channel to channel timing calibration of readout electronics is provided by the T0CAL system. Pulses are injected at the inputs of preamplifiers mounted at the end of each cell with four sense wires. These pulses mimic the characteristics of an electron avalanche on a wire and are processed in the usual way by the subsequent readout electronics. By knowing precisely the time of the pulse injection and measuring the arrival time of the signal as recorded by the corresponding TDC channel, one determines directly the propagation delay from preamplifier to final readout, including channel to channel variations due to distributions of stop signals to individual TDC channels. During offline reconstruction, these propagation delays are subtracted from measured values in determining electron drift times .

Critical to this calibration procedure are stable injection pulses with short rise times requiring fast electronics and high performance, low loss transmission cables between the counting house and the detector. All T0CAL electronics along the path of timing signals are Motorola ECLinPS or ECLinPS Lite logic gates with typical rise times of 250 ps and 300 ps respectively. Signals are fanned out in three stages. The first stage has the longest span $(64 \mathrm{~m})$ for which we use RG213 coaxial cables; the second stage is $27 \mathrm{~m}$, of which $24 \mathrm{~m}$ consists of RG58 coaxial cables and $3 \mathrm{~m}$ (lying in a confined region of limited space) consists of RG174 coaxial cable; the third stage within chamber modules consists of 2-3 m RG178 cables. All electronic channels and long cables were individually calibrated before or during installation. Samples of the shorter cables (less than $4 \mathrm{~m}$ ) were measured to have rms propagation delays better than 120 ps. In addition, a parallel direct return path for all timing signals has been provided, which allows a direct in situ check of absolute timing precision. This return system, independent of the readout electronics, has verified the absolute precision in the T0CAL injection system to $\leq$ $500 \mathrm{ps}$ and a relative channel to channel precision $\leq 300$ ps. Fig. 18 shows an example of such data for one chamber. These calibrations are performed daily and automatically correct shifts from replaced modules.

\subsection{High Voltage System}

A high voltage system supplying the four different voltages to each of the 6144 drift chamber cells of the detector, was designed and built to fulfill the following requirements:

- easy and fast way of disconnecting high voltage of a single cell in case of bad insulation, high dark current or broken wire.

- remote current control of each chamber layer for each of the four voltages and easy and fast way of disconnecting a given layer in a case of unrecoverable overcurrent in a given cell or group of cells.

- online measurement and control of all voltages and currents drawn by the chambers from a given channel of the high voltage power supplies.

- capability to supply the chambers inside the L3 magnet with high voltages which are different from those supplied to the chambers outside.

Voltage lines of 16 cells are grouped into a $3 \mathrm{~m}$ long multiwire high voltage cable (16 conductors) connected to a patch panel located near the chamber. A cable carries only one voltage (sense, cathode, field, or guard), so the four different voltages are supplied in parallel 
from the patch panel by four cables, for a total of 16 cables for the 64 cells in a chamber. The individual connections at the patch panel are removable ${ }^{4)}$.

The high voltages distributed by the patch panels are routed through up to $70 \mathrm{~m}$ long multicore cables to a control system which is physically accessible during data taking. This control system is just an extension of the system used for the central muon detector, and allows permanent monitoring of the current drawn by the chamber cells. The total current drawn by a group of 16 high voltage lines is monitored by a liquid crystal display cell which will light up if the current exceeds $2 \mu \mathrm{A}$. The high voltage connection to this group can then be disconnected. During controlled access to the patch panels, the individual cell drawing the current can be easily identified and disconnected from the high voltage distribution.

The distribution scheme described above requires three CAEN SY127 mainframes equipped with a total of 24 modules (four sources per module) and monitoring facilities. To protect the chambers, all modules operate in a current limited mode of $5 \mu \mathrm{A}$. Whenever this limit is exceeded, the system automatically reduces the high voltage until the current is lower than the limit set.

\subsection{Gas System}

The drift chambers use a non-flammable gas mixture containing $4 \%$ Isobutane, $10 \%$ Carbon Dioxide and $86 \%$ Argon. The total gas volume is about $100 \mathrm{~m}^{3}$ for the 96 chambers and the working condition is 2 mbars above the atmospheric pressure. Each chamber has a gas flow of $160 \mathrm{l} / \mathrm{h}$ which gives a total flow of $15 \mathrm{~m}^{3} / \mathrm{h}$ for all chambers. The gas mixture is circulated through the chambers in a closed circuit with about $5 \%$ of new gas added and $5 \%$ of the old gas removed. This dilutes any micro air leaks and keeps the level of Nitrogen in the gas mixture below $500 \mathrm{ppm}$.

Gas composition is controlled and monitored by two infrared gas analyzers, one for Isobutane, the other for Carbon Dioxide, communicating via an ADC to a VME crate controlling the three mass flow controllers which regulate the individual flows.

$\mathrm{RPC}$ counters use a flammable gas mixture of $38 \%$ Isobutane, $4 \% \mathrm{C} \mathrm{Br} \mathrm{F}_{3}$, and $58 \%$ Argon. The total gas volume is about 1150 liters for 64 chambers. A typical flow rate for one set of chambers is $8 \mathrm{l} / \mathrm{h}$, which gives a total flow of $260 \mathrm{l} / \mathrm{h}$. Working pressure in the chambers is in the range 2 to 3 mbars above atmospheric pressure. A hard wired alarm system is implemented to stop the gas system and switch off all high voltage in case of an alarm.

\subsection{Temperature monitor system}

Because of the large chamber sizes, thermal expansion effects can become non negligible, degrading the precise knowledge of relative wire positions. All chambers were equipped with one sensor on each of the 4 corners, so that not only absolute values but also temperature gradients could be monitored. FI and FM chambers have 2 additional sensors, centered on the outer side of the top and bottom aluminium covers, respectively. The total system consists of 512 sensors.

Sensors are low cost temperature transducers with very good performance. After calibration, all of them measure the absolute temperature, in the range 15 to $30^{\circ} \mathrm{C}$, with a precision of $\pm 0.3^{\circ} \mathrm{C}$. Non linearity is smaller than $0.05^{\circ} \mathrm{C}$, and long term stability better than $0.1^{\circ} \mathrm{C}$.

Temperature values are monitored periodically during L3 general data taking periods. Sensor drivers were implemented in VME cards, each corresponding to 32 multiplexed sensors.

\footnotetext{
${ }^{4)}$ Connector type MSTB 2,5/4-STZ-5,08 produced by PHOENIX.
} 
The monitoring control software runs on a standard OS9 VME crate, ensuring that relevant temperature information is transferred to the L3 general data base system.

\section{$5 \quad$ Trigger RPCs}

\subsection{The RPC System Layout}

Outside the magnet door, between the FM and FO drift chambers, two planes of RPCs are placed in order to generate a fast level-1 trigger for particles coming from the interaction point (see Fig. 1). The choice of this kind of detectors has been motivated by their fast response, good time resolution, flexibility in segmentation and low cost in production. Their use in this system is the first large scale application for counters completely built and assembled by industry. The total system consists of 192 double-gap RPCs of trapezoidal shape, in three different sizes, covering a total area of more than $300 \mathrm{~m}^{2}$.

A single-gap RPC is made of two bakelite plates (2 mm thick) kept at a constant distance of $2 \mathrm{~mm}$ by a $10 \mathrm{~cm}$ pitch grid of round PVC spacers $(\phi \simeq 1 \mathrm{~cm})$. The gap between the two bakelite plates is filled with a gas mixture of Argon, Isobutane and $\mathrm{CBrF}_{3}$ (58:38:4). The resistivity of the bakelite plates used for this application is $\rho \simeq 2 \cdot 10^{11} \Omega \mathrm{cm}$, a value which ensures low noise and full efficiency in presence of the LEP particle flux outside the magnet door. The outer surfaces of the bakelite plates are painted with a graphite solution in order to apply the high voltage. A $300 \mu \mathrm{m}$ PVC foil allows the insulation of these planes. A double-gap RPC (Fig. 19) consists of two of these units, with spacers staggered by $5 \mathrm{~cm}$ so that they do not overlap. The readout plane, placed between the two single-gaps, is segmented in strips with $3.1 \mathrm{~cm}$ pitch. Everything is assembled in a rigid structure made of two planes of foam coated with an aluminium foil and four aluminum profiles.

As described in chapter 7, the basic unit of the F/B muon system is a "half octant" module composed of two drift chambers (FM and FO) joint by a truss structure with the two RPC planes mounted on their inner surfaces. The total number of these units is 32 . Each plane of trigger detectors is composed of three RPCs of different size: L (Large), M (Medium), S (Small). To avoid dead areas due to mechanical constraints in the assembly of a layer, the Medium RPC partially overlaps the Large and Small ones. The active area of the detectors is 2-3 mm larger than that of drift chambers, except for the octant center line side where the active areas of the tracking and trigger detectors have the same extension. The readout strips are placed perpendicular to the octant center line and have a length variable from $98 \mathrm{~cm}$ to 216 $\mathrm{cm}$. Each RPC has 32 strips ( 96 for one plane) so that the total number of readout electrodes is 6144 .

\subsection{Electronics and Tests}

Both sides of the readout electrodes are equipped with front-end electronics for a total of 12288 channels which feed 768 boards mounted on the chambers themselves. Each board accepts inputs from 16 adjacent strips and, after amplification and discrimination $(\simeq 60 \mathrm{mV})$, produces shaped outputs (200 ns differential TTL). A fast signal made on each board with the OR of 16 strips is also generated with a threefold fan-out: one output is fed to an 1875 LeCroy TDC for time measurement, one is used for monitoring purposes and the last is used to generate a Super-OR signal for the 96 strips of one plane. The use of signals from both ends of the strips allows to reduce the time jitter of the Super-OR due to the propagation time along the strips. 
Each front-end channel can be checked by a test pulse which also provides a relative calibration (T0 calibration) for the 768 timing channels.

Before being mounted on drift chambers, all RPCs have been tested in a cosmic ray stand using the same gas mixture as in the experiment. The main purpose of this test was to check the performance of all electronics and to measure the efficiency plateau of each detector by powering one gas volume ("gap") at the time. A typical plateau curve is shown in Fig. 20a). Chambers for which a different working voltage was found have been replaced. Fig. 20b) shows the distribution of the $90 \%$ efficiency working point for all chambers tested. The operating point of the chambers has been chosen between $0.3-0.4 \mathrm{kV}$ above the plateau knee. To reduce the number of HV channels, chambers have been grouped by gap in every octant so that only $64 \mathrm{HV}$ channels are needed with only three different HV values. Furthermore, this ensures redundancy in case of failure since the two gaps in each RPC are powered separately.

\subsection{Level-1 Trigger Generation and Data Readout}

The trigger processor which uses RPC data has been designed for a fast identification of muons coming from the interaction vertex. The trigger logic scheme is shown in Fig. 21a). In order to reduce the number of channels, each input to the trigger processor is the logical OR of two aligned strips in the two "half octants" which form an "octant". The TTL differential outputs of these circuits are sent via $60 \mathrm{~m}$ of twisted pair cable to the Zero Suppressor modules [19] which are physically accessible during data taking. Two of these VME units accept inputs from 96 strips of the FM plane and 96 strips of the FO plane of one octant and their status is latched when a strobe pulse is present. In a typical time of $1 \mu$ s the input pattern is processed and the addresses - if any - of bits found "high" are tranferred to a Track Finder module. The strobe pulse to the Zero Suppressor can be chosen between two possibilities: a signal synchronous with the beam crossing (Beam Gate) or a coincidence between the Beam Gate and the coincidence between the two Super-OR signals (FM-FO planes) of any of the 32 "half octants". The first mode of operation accepts data in a time window of $\simeq 200 \mathrm{~ns}$ around the time of physical events while the second one allows a smaller resolving time of $\simeq 50 \mathrm{~ns}$ and has been used for 1995 runs with LEP operating in bunch train mode [20] ${ }^{5}$ )

The Track Finder unit searches if coincidences, in a $96 \times 96$ matrix (Trigger Matrix), are present in elements $A_{i j}$ which define particles coming from the interaction vertex. The pattern of these elements depends on the polar production angle, momentum, magnetic deflection and the multiple scattering in the $0.9 \mathrm{~m}$ thick iron door. The pattern of particles originating from the interaction point has been defined by a Monte Carlo simulation of the detector and is shown in Fig. 21b). During data taking a 11-element-wide road around the infinite momentum correlation is used. This cut rejects electronics noise, cosmics not pointing to the interaction point and beam halo. The efficiency for events from the interaction point is not affected by this cut. The full programmability via the VME backplane of the Trigger Matrix allows to optimize the running conditions with respect to momentum cut, trigger efficiency and trigger rate. The maximum search time of the unit is $\left(200+50 \cdot N_{i} \cdot N_{j}\right) \mathrm{ns}$, where $N_{i}$ and $N_{j}$ are the numbers of hit strips in the FM and FO planes. If a coincidence is found in the programmed pattern, a trigger signal is generated and sent to the Trigger Box where it is correlated to the information coming from other detectors to produce a level-1 muon trigger for the Forward-Backward region.

The complete trigger processor is composed of 16 sub-systems, each formed by two Zero Suppressor units and one Track Finder unit (one for each octant).

\footnotetext{
5) Each beam consisted of 4 trains with 2 to 4 bunchlets each. The bunchlets were separated by 247 ns.
} 
Data words containing the addresses of hit strips in the event are kept in a memory in the Track Finder modules and are transferred, via an ECL differential asynchronous bus, to Fastbus for data acquisition when a general L3 level-1 trigger is present.

\section{F/B Toroids}

\subsection{Construction}

When closed, the doors of the L3 magnet each form an octagonally shaped torus. A conductor was wound between the inner and outer radius of the door. There are 36 turns per side (see Fig. 22). The water cooled coils are excited by $6300 \mathrm{~A}$ with a $15 \mathrm{~V}$ voltage drop, yielding a nominal power of $94.5 \mathrm{~kW}$. The forward and backward toroidal windings are connected in series to a power supply. The latter is placed on top of the L3 magnet via a bus-bar system. Each turn weighs $140 \mathrm{~kg}$ and the total weight of the aluminium conductor is $11 \mathrm{t}$ for the 2 poles and the bus-bar. It should be noted that a tangential magnetic force of $320 \mathrm{daN} / \mathrm{m}$ is acting on the conductor and the bus-bar system. To provide enough space for the new coils between the poles and the barrel yoke, the doors were moved out by $11 \mathrm{~cm}$.

The coils are made from hollowed aluminum with a cross section of $125 \times 40 \mathrm{~mm}^{2}$. The conductors are cooled by demineralized water inside the conduit. L-shaped half-turns were lowered into the underground area of L3. They were connected by MIG welding ${ }^{6)}$ on the doors to create the windings. The interconnections between the toroidal coils on either side of the main magnet are such that muons of the same charge have the same acceptance on both sides.

This type of construction minimizes the construction time in the underground area, allowing the assembly during a 4 month LEP shut-down. After the welding of the L-shaped half-turns, the insulation was assembled in situ. Halogen free sleeves were slipped over the conductors, and a glass fiber coated tape was wrapped around the coils. Between the coil and the door a foam plate of $10 \mathrm{~mm}$ thickness was inserted for thermal insulation.

During normal running, the coils which are electrically connected in series dissipate a total power of $189 \mathrm{~kW}$. For the cooling 9 turns are connected in series. The total system, including the connecting bus-bars, has 9 independent cooling circuits. The maximum temperature of the system is about $40^{\circ} \mathrm{C}$.

Because of the high current, the power supply is positioned at a minimum distance to the magnet. The power supply is made of two units in parallel which had been recycled. To avoid water losses into the detector, they have no water cooling. The power supplies are voltage regulated.

To protect the inner drift chambers against the heat dissipation of the coils, an active water shield is installed with a separation of $10 \mathrm{~mm}$ of insulating foam against the coils. With 2 circuits per pole and $20 \mathrm{l} / \mathrm{min}$, the water temperature increase can be restricted to $3^{\circ} \mathrm{C}$.

\subsection{Magnetic field measurements}

The determination of magnetic fields inside iron is not straightforward. We have used two methods: One is via the induction in coils wrapped around the iron when ramping the magnet current. The other one uses hall plates installed in the slit between the halfs of the pole pieces. The main purpose of these hall probes was to measure the field direction. Fig. 23 shows on the

\footnotetext{
${ }^{6)}$ Metall Inert Gas welding
} 
left the position of 5 induction coils and the position of the hall plates. One more induction coil was installed on the right hand side to check the field symmetry.

Within the acceptance of the forward/backward spectrometer the measured average field is $1.24 \mathrm{~T}$, given by coil \#2. It drops to about $0.8 \mathrm{~T}$ at larger radii.

\subsection{Magnetic field calculation}

Because the field distribution in the iron is not uniform and cannot be measured in detail, a field map was calculated with the 3 -D program TOSCA [22]. The right hand side of Fig. 23 shows the orientation of the magnetic field vectors inside of the iron as calculated with the TOSCA program. One observes a superposition of the toroidal field ( $\Phi$-direction) and the solenoidal return field (R-direction). Since the pole pieces contain air gaps which cannot be measured well, the amount of air gaps was a free parameter in the calculation. The calculated field map was adjusted to the $1.24 \mathrm{~T}$ measured by the induction method.

Fig. 24 shows the toroidal and solenoidal component as function of the distance to the beam axis $\mathrm{R}$ at $\Phi=0^{\circ}$. The field values were averaged over all $\mathrm{Z}$ values inside of the pole piece. The toroidal component sets in at the pole piece $(\mathrm{R}=240 \mathrm{~cm})$. The drops of the toroidal component at $\mathrm{R}=260 \mathrm{~cm}$ and $420 \mathrm{~cm}$ are due to air slots in the pole piece. Since the acceptance of the spectrometer covers only $240 \mathrm{~cm} \leq R \leq 420 \mathrm{~cm}$, the air gaps were partially filled up with additional iron in this region to enhance the toroidal component. This together with the stronger saturation by the return flux of the solenoid explains the smaller toroidal component in the outer half $(\mathrm{R}>420 \mathrm{~cm})$.

\section{$7 \quad$ System assembly and tests}

Drift chambers and RPCs were integrated at CERN into modules before mounting into the L3 experiment. In this section we describe the last steps of system assembly and the final tests performed prior to installation. We also describe the $\mathrm{F} / \mathrm{B}$ muon detector alignment, both internally and with respect to the rest of the detector.

\subsection{Modules assembly}

The two outer layers of the $\mathrm{F} / \mathrm{B}$ detector are comprised of prealigned modules to simplify installation and alignment on the magnet doors. Modules consist of one FM and one FO chamber with RPCs attached to the inner surfaces of the chambers (Fig. 25). They were assembled on a special stand which aligns the chambers to an accuracy of $100 \mu \mathrm{m}$. The support structure between the two chambers is a truss type frame assembled with accurate jigs to ensure the constant chamber separation and keep the chambers parallel.

\subsection{Tests and Spatial Resolution}

Chamber performance and quality of track reconstruction was tested in all modules with cosmic rays. The data sample for each module typically consisted of $10^{6}$ events recorded with no magnetic field with trigger from the RPCs in the module. Tracks were selected offline to be parallel to the $\mathrm{X}$ and $\mathrm{W}$ wire planes to within $\left|\theta_{x}\right|<20 \mathrm{mrad}$. The inclination in the Y-Z plane was not constrained. Tracks having only one hit on each of the signal wires were reconstructed using a simple time-to-distance relation given by $x=x_{0} \pm v\left(t-t_{0}\right)$, where $v=51.15 \mathrm{~mm} / \mu \mathrm{s}$ is 
the drift velocity, $\mathrm{t}$ is the drift time and $t_{0}$ is the time offset corrected for each wire including the electronic delay and the time of flight of the track. By selecting tracks perpendicular to the drift direction, second-order effects such as angular dependence of the drift time or the inductive cross-talk between wires could be neglected. The left-right ambiguity was resolved by the half-cell offset between the $\mathrm{X}$ and $\mathrm{W}$ layers.

The accuracy of track reconstruction is shown in Fig. 26. For this plot the tracks are fit using only the $4 \mathrm{X}$-wires in the top and bottom chambers and are required to be on the same side of the wire plane in both chambers. This choice allows to study the reconstruction accuracy independent of the position error introduced by the time-uncertainty of the RPC trigger. The residual distribution in Fig. 26a is fit with a gaussian of width $\sigma=200 \mu \mathrm{m}$. This translates to a single-wire position resolution of $230 \mu \mathrm{m}$. A tail due to multiple-scattering is also observed. The dependence of $\sigma$ on drift distance is shown in Fig. 26b and is consistent with diffusion broadening.

The offset between $\mathrm{X}$ and $\mathrm{W}$ layers implies that - for perpendicular tracks - the sum of the drift times in the two layers is a constant, independent of track position. This feature allows a determination of the arrival time of a track with respect to the trigger. It can be used, for example, to associate tracks with closely spaced beam bunches in a collider. For small angles $\theta_{x}[\mathrm{mrad}]$ the average drift-time sum of a chamber (FM or FO) is given by:

$$
\Sigma_{F M, F O}=\frac{1}{4}\left(\Sigma t_{x}+\Sigma t_{w}\right) \pm 1.20 \frac{\mathrm{ns}}{\operatorname{mrad}} \theta_{x}
$$

where $t_{x}$ and $t_{w}$ are the 4 drift-time measurements (ns) in the $\mathrm{X}$ and $\mathrm{W}$ layers respectively, and the sign of the angular term depends on the sign of $\theta_{x}$ and which side of the wire plane the track is in. The average time sum for the 2-chamber package is given by $\frac{1}{2}\left(\Sigma_{F M}+\Sigma_{F O}\right)$. In tests with cosmic rays described here, the time sum resolution is strongly influenced by the trigger timing uncertainty. In order to cancel this contribution we plot instead in Fig. 27 the difference $\frac{1}{2}\left(\Sigma_{F O}-\Sigma_{F M}\right)$ which has the same resolution as the sum would have with a precise trigger. The distribution is best fit by 2 gaussians, the narrower one giving a resolution of $\sigma=2.4 \mathrm{~ns}$. It is interesting to note that this resolution allows to distinguish, at the $3 \sigma$ level, tracks that traverse the package in the opposite direction.

Additional checks of individual chamber performance were carried out by comparing the two segments of a track reconstructed in the $\mathrm{X}$ and $\mathrm{W}$ layers. In particular, requiring that the two segments intersect at the midplane between the two layers provides a strong consistency check of the reconstruction parameters including the drift velocity and the $t_{0}$ offset. Fig. 28 shows the difference of intercepts of the 2 segments at midplane. As an example, the effect of a $20 \mathrm{~ns}$ shift in $t_{0}$ is shown as well. This shift is equivalent to a change in the drift velocity of approximately $2 \%$ and cannot be distinguished from it. This method allows for a sensitive monitoring of the stability of the velocity and time offset for individual chambers.

\subsection{Attachment to Doors}

Pre-aligned fixed support feet were used to attach the FM-FO modules to the magnet door. FI chambers are supported by adjustable feet which allow to adjust the position of the FI chamber in order to align it with respect to its corresponding FM-FO module. The alignment procedure and the obtained accuracy will be described in the next section.

The separation distance in $z$ between FI and FM, and between FM and FO, was measured with a special caliber at the three points where the chambers are attached to the magnet door. The accuracy of these mechanical measurements is of the order of $200 \mu \mathrm{m}$. 


\section{$7.4 \quad$ Alignment systems}

Fig. 29 gives an overview of the alignment system of the F/B muon detector. Two sub systems are needed. In the S-region, the essential part is the alignment of the FI chamber with respect to the barrel muon chambers MI and MM. There, the alignment is critical to achieve the optimal muon resolution. In the T-region only the relative alignment of the chamber layers FI, FM and FO is important. A detailed description of the system components, the calibration and tests performed, as well as the procedure followed to install them onto the chambers and to align the half octants can be found in [23].

\section{Relative alignment between FI, FM and FO layers: T-region}

The important coordinates to be monitored in order to achieve the required alignment are $x$ and $y$. A RASNIK [24] system, similar to the one used in the barrel muon octants, was implemented. Holes were drilled through the magnet doors so that optical straightness monitors could line up the three chamber layers. The optical monitors include an LED on FI, a lens on FM and a four quadrant diode on FO.

This new RASNIK system has a range of $\pm 2.5 \mathrm{~mm}$ and an accuracy of about $50 \mu \mathrm{m}$ in both $x$ and $y$. This accuracy is more than sufficient, as the momentum resolution in the T-region is limited by the multiple scattering through the magnet door, which is of the order of $1 \mathrm{~mm}$ at $45 \mathrm{GeV}$.

\section{Alignment with respect to the central detector: S-region}

There are three steps involved in the alignment of the forward backward system with respect to the central detector:

The first one is to get marks, or reference blocks, onto the FI chambers. These marks should contain all relevant information about internal chamber geometry. In order to define the position of each FI with respect to the central detector 6 coordinates have to be measured and therefore 6 reference marks were needed. We chose them to be three reference plates providing contact surfaces to measure $z$, and three reference blocks used as reflectors by proximity sensors (described below) to measure 2 values of $x$ and 1 value of $y$ (Fig. 30). Accurate positioning of the blocks which provide the $x$ and $y$ reference surfaces was accomplished by means of specially developed devices including laser interferometry systems and very precisely machined pieces [23]. From these reference surfaces, the location of the first $\mathrm{X}$ wire plane is known with an accuracy of $15 \mu \mathrm{m}$, and the the first $\mathrm{Y}$ wire plane with an accuracy of $50 \mu \mathrm{m}$. The $z$ reference plates are accurate to $300 \mu \mathrm{m}$ in defining wire positions along the $z$ coordinate.

The second step is to get the barrel muon detector geometry transferred to reference blocks close to the FI chamber marks when the magnet doors are closed. Unlike the FI marks, these reference blocks attached to the barrel octant structure have $\mathrm{L}$ shaped pieces with precision holes to hold proximity sensors. As explained below, these sensors were calibrated to measure the distance to a given surface taking as origin a precision pin, incorporated in the sensor and made to match the precision holes of the reference block. It is then the location of these holes with respect to the muon chamber wires which is relevant. Achieving the expected accuracy here required careful measurements in every octant during the installation:

- For the measurement of the $x$ position of these holes, a Laser Beacon system was used [25]. Each octant already has four diode arrays located between MM and MO chambers, in 
both ends (magnet door side MD, and interaction point side IP), whose position with respect to the first wire plane of each chamber is accurately known. As described in [25], a laser on a rotating pentaprism, or laser beacon, defines a plane and illuminates the four diode arrays. This procedure was used to measure the torsion on all muon barrel octants during the initial alignment phase of the central detector. For the actual F/B detector alignment, two additional laser beacon sensors were attached to the precision holes of the sensor holders. The central octant plane can then be extrapolated there and the coordinate obtained can be compared with the one measured by the laser beacon sensors when the laser hits on them, thus yielding the $x$ alignment with an accuracy of $50 \mu \mathrm{m}$.

- For the calibration of the $y$ position, we have used the octant $y=0$ reference surfaces which are located below MM support bars. A laser diode fixed to one of these surfaces (on the IP side) emitting light towards the other one, and a diode pixel array sensor attached to the surface at the MD side, define the $y=0$ plane. As described in [23], a simple procedure allows to get the $y$ position of the precision hole with an accuracy of $50 \mu \mathrm{m}$.

- For the reference blocks to measure the $z$ coordinates, the required accuracy was not very tight. Therefore, measurements with standard instruments by the CERN survey team were used with an accuracy of $500 \mu \mathrm{m}$.

Finally the third step involves the continuous monitoring of the distances between FI marks and the octant reference blocks. This is done by special proximity sensors held by the octant reference blocks. Two different types of proximity sensors were used. The $x$ and $y$ displacements of chambers are monitored by laser triangulation devices ${ }^{7)}$. This method has the advantage of allowing a contact free distance measurement. The $z$ monitoring is done by linear potentiometers ${ }^{8)}$, making a mechanical contact with the FI $z$ reference marks. The proximity sensors are calibrated with respect to the above mentioned precision pin. The resolution is in the range of 20 to $50 \mu \mathrm{m}$ depending on the measured distance. The readout system is based on VME ADCs permitting a readout of all sensors every two minutes. The data is logged into the L3 online data base every two hours for any movement measured greater than $50 \mu \mathrm{m}$. Fig. 31 shows a typical distance measurement of a triangulation sensor. The movements of about 500 $\mu \mathrm{m}$ demonstrate the importance of the alignment system.

The information provided by the proximity sensors is read out from the data base and used for offline reconstruction on an event by event basis. The geometry which defines the positions of the FI/FM/FO chambers at the time when the event was taken is then calculated and used to reconstruct the muon trajectory. Taking into account all effects, we estimate that the achieved accuracy in the sensor wire coordinates is about $70 \mu \mathrm{m}$ in $x, 150 \mu \mathrm{m}$ in $y$ and $580 \mu \mathrm{m}$ in $z$.

\section{Performance at LEP}

The performance of the chambers and the RPCs after installation has been evaluated on data taken with the L3 detector at LEP in 1995, using $e^{+} e^{-} \rightarrow \mu^{+} \mu^{-}(\gamma)$ events.

\footnotetext{
${ }^{7)}$ Type OPTIMESS 30LP by ELAG AG, Winterthur.

${ }^{8)}$ Type CR18-50K by DATAMEGA S.A., La Chaux de Fonds.
} 


\subsection{Muon track reconstruction}

Energetic muons produced at the $e^{+} e^{-}$interaction region under polar angles $24^{\circ}<\left(180^{\circ}-\right) \Theta<$ $44^{\circ}$ are measured in the $\mathrm{F} / \mathrm{B}$ chambers, but also traverse one or more barrel muon chambers. Therefore, the track reconstruction software for the $\mathrm{F} / \mathrm{B}$ chambers is closely linked to that of the barrel muon chambers [26].

In the S-region, the muon trajectory through the L3 solenoidal field is measured both in two precision $(\mathrm{P})$ chambers in the barrel muon detector and in one FI chamber (see chapter 1.2 and Fig. 1). In the T-region, a muon is measured in one $\mathrm{P}$ chamber in the barrel, in one FI chamber inside the solenoid volume, and in the FM and FO chambers outside of the toroidal doors.

In the next paragraphs we shortly describe the track reconstruction procedure. Where the reconstruction procedure needs geometrical constants or time-dependent data (alignment, $t_{0}$ ), this data is read from the L3 database [27].

Initially, the multiplexed TDC data and the recorded hit addresses are matched to each other and combined in order to create candidate hits in the cells. For those cases in which no unambiguous match can be found, all allowed combinations are stored as hit candidates. The drift times are corrected using the wire-by-wire $t_{0}$ values, as determined by the $t_{0}$ calibration system described in section 4.3 , and a global $T_{0}$ that synchronizes the times recorded in the F/B muon system with the absolute LEP bunch crossing time. This global $T_{0}$ is obtained from the data itself using the requirement that track segments reconstructed in the staggered $\mathrm{X}$ and $\mathrm{W}$ layers of the chambers are aligned. The $T_{0}$ values are determined at regular intervals during data taking and are carefully monitored, significant shifts have been observed only after major cable or electronics replacements. Hits in the RPC system are stored in a similar fashion.

The pattern recognition program then tries to find track segments in the individual $\mathrm{Y}, \mathrm{X}$ and W layers of the chambers. A "cell map" converts the drift times of the hit candidates into drift distances along the drift paths. This map is based on calculations of drift paths and drift velocities with the program GARFIELD [28]. Locally in the drift cells, the track segments can be approximated with sufficient accuracy by straight lines, fit through 3 or 4 hits in a single layer of cells. In order to resolve ambiguities arising from left-right permutations, the segments reconstructed in a single projection are iteratively matched and merged within a single chamber, as well as between the FM and FO chambers of a single package.

The coordinates of the hits belonging to the reconstructed segments are corrected using the information on the actual chamber positions given by the alignment systems and geometrical survey and construction data. After this correction, a refit is performed in order to obtain the final segments.

Subsequently, the track segments in the two projections ( $\mathrm{Y}$ and $\mathrm{X} / \mathrm{W}$ ) are combined, and assembled into track candidates by matching them to segments in other $\mathrm{F} / \mathrm{B}$ chambers and segments from the barrel muon detector. Combinations that match are stored as muon track candidates with an initial estimate of momentum and polar and azimuthal angle.

Improved track parameters of the muon candidates are obtained in a fit $[29,30]$ that takes into account the chamber measurements along the track, all material in the detector and the actual magnetic field. Hits in the barrel and endcap scintillator counters and clusters in the RPCs (see section 8.5.2) close to the muon trajectory are assigned to the muon track candidate. By assigning a time to a track, we are able to separate cosmic rays from muons produced in actual $e^{+} e^{-}$interactions, and to identify the bunch crossing. Finally, the track candidate is extrapolated from the muon chambers back to the inner detectors, and matched with a track in the inner tracking chamber (TEC). Using TEC, calorimeter and timing information, the track 
parameters are determined at the vertex, remaining track ambiguities are resolved and the track is classified as either a good muon candidate, a candidate for a cosmic ray, or a possible hadronic punch-through.

\subsection{Characteristics of the data}

In L3, dimuon events are pre-selected on the basis of the presence of at least one muon reconstructed in the muon chambers, or a sufficient number of track segments in the chambers, or an energy cluster in the calorimeters with the characteristics of a minimum ionizing particle. For the results presented here, the sample contains events with at least one reconstructed muon with track segments in the F/B muon chambers, with either an associated hit in the scintillator counters within a time window of $4 \mathrm{~ns}$ around the beam crossing, or an associated track in the inner tracking detector TEC with a radial distance of closest approach to the beam axis of less than $2 \mathrm{~mm}$. In addition, an upper limit is set to the amount of clusters found and energy measured in the calorimeters, and the reconstructed muon momentum is required to be larger than $18 \mathrm{GeV}$. The resulting sample consists of 5864 events, of which $58 \%$ are in the toroidal region. The background of events other than $Z \rightarrow \mu^{+} \mu^{-}$is expected to be a few percent. Fig. 32 displays a dimuon event in the data.

In $e^{+} e^{-}$annihilation at $\sqrt{s}=2 E_{b}$, where $E_{b}$ is the LEP beam energy, the energy of the final state muons is kinematically constrained to be $E_{b}$, when initial and final state radiation are neglected. In practice, radiation gives rise to a tail in the muon energy spectrum towards lower energy. The results presented here have been obtained with data taken with LEP beam energies between 44.7 and $46.5 \mathrm{GeV}$.

In Fig. 33 a, the distribution of the cosine of the polar angle $\Theta$ of the tracks in the selected sample is shown. The full histogram corresponds to all reconstructed tracks with at least one segment in the $\mathrm{F} / \mathrm{B}$ muon chambers, showing the acceptance in $\Theta$ of the whole $\mathrm{F} / \mathrm{B}$ muon chamber system. The hatched area corresponds to tracks having segments in the outer chambers (i.e. the T-region), where the momentum is reconstructed by the determination of the track bending in the toroidal field in the doors. This hatched angular region is not covered at all by the L3 barrel muon chambers.

In Fig. $33 \mathrm{~b}$, the impact points of the reconstructed tracks in the FI chambers are plotted in the $x-y$ plane for the chambers on one particular door. The structure of 16 chambers in 8 octants is clearly visible. Apart from the cracks between the chambers, the track distribution in azimuth $\Phi$ is uniform.

Figs. 32 and 33 show that the reconstruction of muon tracks in the F/B muon system can be performed reliably, indicating that the multiplexing scheme used in the readout works. The track reconstruction efficiency was estimated by extrapolating muon tracks measured in the barrel chambers into the FI chamber. Taking into account the fiducial volume of the F/B chambers (measured in a test set-up with x-rays) and eliminating wires disconnected from high voltage, the combined chamber- and track-finding-efficiency is found to be typically better than $98.5 \%$.

\subsection{Spatial Resolution}

The spatial accuracy of the chambers has been checked in situ at the L3 detector. Muons originating from the $e^{+} e^{-}$interaction region cross the $\mathrm{Y}$ layers of the chambers with a slope $d y / d z$ ranging from 0.4 to 1.0 , whereas the $\mathrm{X}$ and $\mathrm{W}$ layers are crossed with slopes $d x / d z$ 
ranging from 0 . to 0.4 . A straight line is fit through either 4 hits in the $\mathrm{Y}$ layers, or 8 hits in the $\mathrm{X}$ and $\mathrm{W}$ layers. The spatial accuracy of the chambers can then be determined on a single wire basis from the hit residuals, after correction for the statistical wire-by-wire contribution to the fit.

Fig. 34 shows the distribution of corrected residuals for 8 point fits in the $\mathrm{X}$ and $\mathrm{W}$ layers in the FM and FO chambers, for outer wires (wires 1 and 4, closest to the guard strips, as shown for example in figure 6) and inner wires (2 and 3) separately. There is practically no magnetic field in the FM and FO chambers. In these distributions, all tracks with different slopes and locations were used. Fits with a single Gaussian typically give a $\chi^{2} / n d f$ of 2.5 , indicating a poor fit, with a width of $278(264) \mu \mathrm{m}$ for the outer (inner) wires. Since the distributions include tracks crossing the wire planes or the I-beams, where the resolution is worse, we fit the distributions with a sum of two Gaussians. The first Gaussian describes the core of the distributions and contains $85 \%$ of the hits with $\sigma_{\text {core }}=236 \pm 16 \mu \mathrm{m}(225 \pm 16 \mu \mathrm{m})$ for the outer (inner) wires, which agrees with the expectations (section 2.2) and the cosmic ray tests (section 7.2). The second Gaussian describes the tails and contains $15 \%$ of the hits with $\sigma_{\text {tail }}=508 \pm 102 \mu \mathrm{m}(469 \pm 94 \mu \mathrm{m})$ for the outer (inner) wires. The results obtained in the $\mathrm{X}$ and $\mathrm{W}$ layers in the FI chambers are compatible with those obtained in the FM and FO chambers.

In the $\mathrm{Y}$ layers, the resulting resolutions are typically $350 \mu \mathrm{m}$ (single Gaussian fit), which is somewhat worse, due to the fact that tracks cross the $\mathrm{Y}$ layers under much larger slopes.

Combining the individual wire measurements, the typical chamber point resolution is of the order of $100 \mu \mathrm{m}$ in $x$ and $180 \mu \mathrm{m}$ in $y$. The track slope $d y / d z$ is measured with an accuracy of $13 \mathrm{mrad}$ in a single $\mathrm{Y}$ cell (like in $\mathrm{FI}$ ), whereas $d x / d z$ is measured with an accuracy of $3 \mathrm{mrad}$ in the combined $\mathrm{X}$ and $\mathrm{W}$ layers.

Combining the $\mathrm{Y}$ cells in FM and FO, the track slope outside the door is reconstructed with an accuracy of $0.2 \mathrm{mrad}$, which should be compared to an r.m.s. multiple scattering in the magnet door of $3 \mathrm{mrad}$ at $45 \mathrm{GeV}$. Therefore, the spatial accuracy of the chambers does not contribute significantly to the momentum resolution in this region.

\subsection{Momentum Resolution}

In Fig. 35, the distribution of the variable $E_{\text {beam }} /\left(q \cdot p_{\text {muon }}\right)$ is shown for two subsamples of the selected dimuon events. Here, $E_{\text {beam }}$ represents the LEP beam energy, $p_{\text {muon }}$ the reconstructed muon momentum, and $q$ its reconstructed charge. The first sample is reconstructed in the solenoidal region, with $38^{\circ}<\Theta<43^{\circ}$, whereas the second sample is reconstructed in the toroidal region, with $25^{\circ}<\Theta<30^{\circ}$.

Clearly visible are the two peaks at $E_{\text {beam }} /\left(q \cdot p_{\text {muon }}\right)= \pm 1.0$, corresponding to positive and negative muons. The region around $E / p=0$ is populated by tracks with a very large fitted curvature. For these muons the possibility exists to be reconstructed with the wrong charge, we estimate the charge confusion probability to be negligible in the solenoidal region, and approximately $3 \%$ in the toroidal region.

The momentum resolution $\delta p / p$ obtained for muons of $45 \mathrm{GeV}$ momentum in 1995 , as calculated from the selected sample in the $\mathrm{F} / \mathrm{B}$ muon system, is shown as a function of the polar angle $\Theta$ of the track in Fig. 36. The momentum resolution as a function of polar angle $\Theta$ shows the expected behavior (see figure 4), also quantitatively. The resolution $\delta p / p$ ranges from $5 \%$ at $\cos \Theta=0.72$ to $20 \%$ at $\cos \Theta=0.81$ at $45 \mathrm{GeV}$, and equals $30 \%$ in the toroidal region, independent of momentum, which agrees with the multiple scattering limit. 


\subsection{RPC Performance}

The RPC performance has been studied on a similar dimuon sample as described above. More details can be found in reference [21].

\subsubsection{F/B trigger efficiency}

The trigger studies are restricted to the T-region, where the RPCs contribute to the trigger decision. The trigger system in this region is based on the RPCs, the F/B chambers, and one plane of endcap scintillators on either side of the magnet. This allows redundancy and reduces the trigger rate due to the beam background outside of the magnet (where the RPCs are placed).

The trigger logic is divided into a dimuon trigger and a single muon trigger. The dimuon trigger requires the coincidence of two triggers either on opposite sides of the magnet or opposite in $\Phi$ on the same side. In each case, a trigger can be either a RPC trigger or a combined endcap scintillator-F/B chamber trigger. If the two triggers occur on the same side, their octants must be "opposite" in $\Phi$ within a tolerance of \pm 1 octant. The single muon trigger requires the coincidence of triggers from two different detectors in the same octant. All three combinations of RPC trigger, F/B chamber trigger and endcap scintillator trigger are allowed.

The trigger efficiency has been studied using events with at least a trigger different from the $\mathrm{F} / \mathrm{B}$ trigger in such a way as to allow an unbiased evaluation. The measured dimuon trigger efficiency is $\epsilon_{\text {dimuon }}=99.8 \pm 0.4 \%$ and the single muon trigger efficiency is $\epsilon_{\text {single }}=98.7 \pm 0.5 \%$, both averaged over the whole 1995 data sample.

\subsubsection{Spatial resolution and detector efficiency}

When a charged particle crosses an RPC, the discharge in the gas volume induces signals on the strips in a region a few millimeters wide. Discharges in the two separate gas volumes are read by the same electronics. Sloping particle tracks broaden this charge distribution, so a signal can be induced on more than one strip. At the reconstruction level adjacent strips are joined to form a "cluster". A cluster is described by the "center of gravity" of the fired strips and by the multiplicity i.e. the number of fired strips. Since there is a TDC readout on both ends of a strip, the cluster information exists twice. The cluster multiplicity increases with the high voltage applied to the RPC electrodes. For the L3 running conditions ( $\mathrm{HV}=7.5-7.8 \mathrm{kV})$ we obtained an average cluster multiplicity of $\mu=2.10 \pm 0.02$.

Comparing the position ( $y$-coordinate in Fig. 1) of the cluster centroid with the expected position reconstructed using the $\mathrm{F} / \mathrm{B}$ drift chambers, we obtain a global spatial resolution of $\sigma_{y}=7.7 \pm 0.1 \mathrm{~mm}$. This spatial resolution depends on the cluster multiplicity. Selecting events with cluster multiplicity one, two or three we obtain respectively: $\sigma_{y, 1}=6.1 \pm 0.2 \mathrm{~mm}$, $\sigma_{y, 2}=7.8 \pm 0.2 \mathrm{~mm}, \sigma_{y, 3}=8.8 \pm 0.4 \mathrm{~mm}$. The global resolution is then the weighted mean of these specific resolutions.

In the case of full detector efficiency, at least one cluster should be found for each FM/FO plane. However, it is also possible to find more than one cluster in a plane because of the overlap between the Medium RPC and the other two counters (Large and Small, see chapter 5.1). The number of events for which no cluster was found neither in the FM nor in the FO plane provides an estimate of the overall detector efficiency of $\epsilon=99.5 \pm 0.1 \%$. 


\subsubsection{Time resolution and bunchlet identification}

To measure the event timing, the time measured by the RPCs needs to be corrected mainly for three effects:

- different cable lengths and electronic response among channels,

- time of flight of the muons,

- time of propagation of the induced signal along the strips.

The first effect is corrected using the T0 calibration. It consists of pulsing every timing readout channel (Fast-OR of 16 strips) on the front end boards and reading the obtained mean time (T0) at the end of the read-out chain, i.e. on the TDCs. The obtained T0s can differ by several ns and have to be subtracted from the timing data.

The time of flight of the muon can be calculated with enough precision using the impact point on the RPCs extrapolated from the tracks reconstructed by the drift chambers.

To calculate the correction due to the propagation time of the induced signal along the strip we also use the information coming from the F/B tracks, since the RPCs cannot provide the precision required. The length of the strips varies from 96 to $216 \mathrm{~cm}$ corresponding to a time spread of 5-10 ns with a propagation of $1 / v_{p}=4.8 \mathrm{~ns} / \mathrm{m}$ as estimated from the data.

After having taken into account all these corrections the time resolution is $\sigma=2.43 \pm 0.03 \mathrm{~ns}$ as shown in Fig. 37, where also the inter-bunchlet time has been subtracted.

For LEP runs in bunch train mode, the RPCs also provide the bunchlet identification, i.e. in which bunchlet the event occurred. This can be checked with respect to other identifications given by different detectors and with respect to the time of the RPC event. Fig. 38 shows the RPCs time distribution, without subtracting the inter-bunchlet time, versus the bunchlet number identified by the RPCs trigger logic. Moreover, the RPCs bunchlet identification is found to be in good agreement with that from other detectors.

\section{Summary}

A F/B muon spectrometer comprising 96 large drift chambers of a new design was constructed in 2 years. The chambers have proven to be easy in construction, reliable in operation, and accurate. The four-wire track segments permit robust reconstruction. The double layer structure provides self calibration and allows bunch tagging. Multiplexing was successfully demonstrated. Large area Resistive Plate Counters are used for the first time as trigger counters. The RPCs have shown high efficiency and good time resolution. The F/B muon spectrometer operated immediately and achieved the expected momentum resolution.

\section{Acknowledgement}

We would like to acknowledge the contribution to the construction of the $\mathrm{F} / \mathrm{B}$ detector by the Physics Department of the University of Geneva, Switzerland. In addition, we would like to thank our colleagues from the L3 collaboration led by Prof. S.C.C. Ting for discussion and encouragement. The administrative support of $\mathrm{R}$. Vonesch is appreciated. We acknowledge the support from the Deutsches Bundesministerium für Bildung, Wissenschaft, Forschung und 
Technologie, de Stiching voor Fundamenteel Onderzoek der Materie (FOM) for NIKHEF, the Board of the Swiss Federal Institutes of Technology (BSIT), the Comisión Interministerial de Ciencia y Tecnología in Spain, the Schweizerischen Nationalfond in Switzerland and the Department of Energy (DOE DE - AC02 - 76ER03069) and the National Science Foundation in the US. 


\section{References}

[1] Proceedings of the workshop on "Physics at LEP 2", CERN yellow report 96-01 (1996)

[2] B. Adeva et al, NIM A289(1990),35;

B. Adeva et al, NIM A323(1992),109.

[3] H. van der Graaf et al.: The Honeycomb Strip Chamber, Proceedings of the ECFA Study Week on Instrumentation Technology for High-Luminosity Hadron Colliders, Barcelona, 14-21 September 1989, CERN 89-10, ECFA 89-124, vol.2, 24 November 1989;

H. van der Graaf et al., NIM A307 (1991) 200.

[4] I-beam chambers with four signal wires:

U. Becker, Forward Muon Detector, L3 collaboration notes 999 (1991), 1955 (6/1991), and report nb. 1083 (1991);

For earlier versions with a single wire see:

U. Becker et al., NIM A128 (1975), 593;

M. Cerrada et al., NIM A263 (1988), 343.

[5] U. Becker et al., NIM A180 (1981), 61;

P. Duinker et al., NIM A201 (1982), 351.

[6] L3 Collaboration, O. Adriani et al., Phys. Rep.236 (1993), 1- 146.

[7] Included in: U. Becker et al., Compilation of Gas Parameters MIT-LNS 94 (1994);

also WWW http://www-lns.mit.edu, select "Experiments and Collaborations" and "Gas R\&D"

[8] U. Becker et al., NIM A351 (1994), 583 and NIM A361 (1995), 401.

[9] U. Becker et al., NIM A360 (1995), 476.

[10] WIRCHA A program package for wire chamber simulation. H. Fehlman (1989) ETH-Z.

[11] J. Berdugo et al.: Precision templates for gluing wire holding pieces to chamber frames, L3 F/B technical note (September 1992).

[12] R. Bock et al., NIM A336 (1993), 128.

[13] R. Bock et al. NIM A345 (1994), 256.

[14] P. Rewiersma, The L3 Wire-Amplifier, NIKHEF 1986.

[15] F.D. Waldhauer, Wideband feedback amplifiers, IRE Transactions on Circuit Theory $4,5 / 1957$.

[16] J.C.S. Richards, Very wideband amplifiers using transistor feedback pairs, Proc. IEEE 117, $10 / 1970$.

[17] G. Viertel, FB muon chamber front end electronics, Internal Report ETH-Z (November 1994). 
[18] T.Moers et al., Nucl. Inst. and Meth. A 345 (1994) 474.

[19] A.Aloisio et al., IEEE Transactions on Nuclear Science VO1. 41, No. 1, February 1994.

[20] Proceedings of the fifth workshop on LEP performance, CERN SL/95-08 (DI), Chamonix, January 13-18, 1995.

[21] A. Alosio et al., proceedings of the "International Workshop on the RPC in Particle Physics and Astrophysics", Pavia 1995;

[22] J. Simkin and C.W. Towbridge, Int. Jour. Num. Meth. Eng., 14 (1979).

[23] J. Berdugo et al.: The alignment of the forward backward muon chambers, L3 note nb. 1909 (1996).

[24] P. Duinker et al., NIM A269 (1988), 544.

[25] R. Fabretti et al., NIM A280 (1989), 13;

M. Fabre : The dimuon mass resolution of the L3 experiment at LEP, ETH thesis nb. 9696 (1992) 39.

[26] See for example the program description in

T. Foreman, Bottom Quark Production at the Z Resonance, thesis University of Amsterdam (1993), unpublished.

[27] B. Adeva et al., Nucl. Instr. and Meth. A309 (1991), 318

[28] GARFIELD, a drift-chamber simulation program, CERN Program Library W5050

[29] V. Innocente and E. Nagy, Nucl. Instr. and Meth. A324 (1993), 297

[30] V. Innocente, M. Maire and E. Nagy, the GEANE program, CERN Program Library W5013-E

\section{List of Figures}

1 Side view of the F/B detector of L3 showing inner (FI), middle (FM), and outer (FO) drift chambers and RPCs for triggering. The two angular regions, $\mathrm{S}$ and $\mathrm{T}$, are indicated. . . . . . . . . . . . . . . . 28

2 Simulated $\mathrm{e}^{+} \mathrm{e}^{-} \rightarrow \mathrm{W}^{+} \mathrm{W}^{-} \rightarrow \mu \nu_{\mu}+$ two jets event in the L3 detector. The direction of the undetected $\nu_{\mu}$ is indicated as well. The forward detector is essential for muon detection. . . . . . . . . . . . . . . . 29

3 Schematic view of the L3 F/B muon detector. . . . . . . . . . . . . 30

4 Expected muon momentum resolution as a function of polar angle. . . . . . . . 31

$5 \quad$ Drift chamber of 3 layers made of cells with 4 signal wires interspersed with 5 field wires and guard strips above and below the wire plane. . . . . . . . . . 32 
6 On the left, the equipotential configuration of a drift cell with 4 signal wires is shown. On the right, drift field trajectories are shown. . . . . . . . . .

7 a) Drift velocity along the electrical field for different $B$ field values. The upper curve shows, for $\mathrm{B}=0$, the effect of a $1 \% \mathrm{~N}_{2}$ contamination. b) Lorentz deflections as a function of $\mathrm{E}$ for $\mathrm{B}$ field values of $0.5 \mathrm{~T}$ and $0.55 \mathrm{~T}$. . . . . . . . . . .

8 Monte Carlo simulation of: a) Spectrum of arrival times for two wires (on the left an outer, on the right an inner wire) for a $26 \mathrm{~mm}$ drift distance. b) Jitter in the arrival of the $8^{\text {th }}$ electron. c) Resolution expected from the time jitter for various drift distances. ......................

9 View of a chamber. The upper part gives the interior structure of the $\mathrm{W}, \mathrm{X}$ and $\mathrm{Y}$ layers. The lower part depicts the corresponding chamber frame with the holes for the wire planes. . . . . . . . . . . . . . . . .

10 Detailed sketch of an endframe showing a) holes where plastic inserts will be placed, b) plastic insert already in place showing the glass cylinder, c) one which has wires on $\mathrm{Cu}$-Te blocks, and $\mathrm{d}$ ) one cell gas sealed and ready for electrical

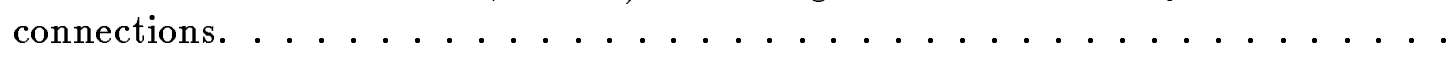

11 a) Sketch of the precision template used to position glass cylinders which define wire plane locations. b) Residuals showing template accuracy. . . . . . . . . .

12 A schematic drawing of the wiring machine (only one of the nine wires shown), with closeups of the crimping tool with fixation block and different types of $\mathrm{Cu}$-Te cubes.

13 Mechanical tension deviations from the design values for (a) field and (b) signal wires. Shadowed are the critical long wires. The dashed lines indicate the design tolerance of $\pm 4 \%$.

14 Typical counting rates using cosmics in one FB chamber. Rates are proportional to wire lengths, which explains the pattern observed in the Y layer (slow increase from cell number 1 to 27 ) and in the $\mathrm{W}$ and $\mathrm{X}$ layers (increasing from cell number 1 to 11 and staying constant for the rest of the cells. . . . . . . . . . . . 40

15 Preamplifier circuit. . . . . . . . . . . . . . . . . . 41

16 Multiplexer of $8: 1$ channels with address recording. . . . . . . . . . . . 42

17 Readout schematic. . . . . . . . . . . . . . . . . 43

18 TOCAL test as performed to one of the chambers providing the relative channel to channel timing calibration for all wires in all layers. . . . . . . . . . . 44

19 Cross section of a double-gap RPC (not to scale). . . . . . . . . . . . . . 45

20 a) Typical plateau curve; b) Distribution of the $90 \%$ efficiency working point for all tested chambers. . . . . . . . . . . . . . . . . . 46

21 a) Trigger logic; b)Monte Carlo simulation of the Trigger Matrix for muons with different momenta. . . . . . . . . . . . . . . . . . . 46

22 End view of the L3 magnet with the 36 turns of one toroidal coil. . . . . . . . . 47

23 Left side: Location of field measuring devices. Right side: magnetic field vectors inside the iron according to a TOSCA calculation. . . . . . . . . . . . .

24 Calculated toroidal field strength as function of the distance to the beam axis $\mathrm{R}$ at $\Phi=0^{\circ}$, averaged over the thickness of the door which extends from 2.4 to $5.9 \mathrm{~m}$. The dips correspond to air gaps. The solenoidal part in the air and its return flux in the doors is shown as dotted curve. . . . . . . . . . . . . 49

25 Schematic view of module assembly. . . . . . . . . . . . . . 50 
26 (a) Distribution of the fit residuals of the cosmic ray tracks. (all selected events, see text) The gaussian fit has a standard deviation of $200 \mu \mathrm{m}$. (b) Dependence of $\sigma$ on the drift distance. The curve is $\sigma=87+21 \times \sqrt{\text { distance }(\mathrm{mm})} \mu \mathrm{m}$. . . . 51

27 Distribution of the difference of the mean drift-time sums of the two chambers forming a module (see text). . . . . . . . . . . . . . . . . 52

28 The distance between the two segments of a track fitted in the $\mathrm{X}$ and $\mathrm{W}$ layers measured at the midplane (solid histogram). Tracks on the right (a) and left (b) sides of the $\mathrm{X}$-wires are shown separately together with a gaussian fit. The shaded histogram is obtained when the same tracks are analyzed with a time

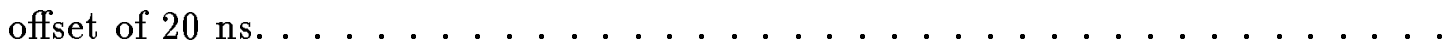

29 Overview of the alignment system in the $\mathrm{F} / \mathrm{B}$ region, not to scale. The alignment consists of 4 steps: (A) positioning of the sensor's reference marks with respect to the barrel chamber wires, (B) a distance measurement by a sensor with respect to FI surfaces, $(\mathrm{C})$ positioning of these reference surfaces with respect to the FI chamber wires and (D) the internal alignment of the three $\mathrm{F} / \mathrm{B}$ layers by means of a RASNIK system. . . . . . . . . . . . . . . . . 54

30 A front view of an FI chamber showing the position of the reference marks for $x, y$ and $z \ldots \ldots \ldots \ldots \ldots \ldots$

31 Distance between the muon barrel and the reference marks of a FI chamber recorded by a triangulation sensor over 10 days. The top bar indicates the status of the L3 magnets. A difference in chamber position of about $500 \mu \mathrm{m}$ can be seen for the two periods with both magnets powered. . . . . . . . . 56

32 Side view of a dimuon event in data taken at LEP. . . . . . . . . . . . . 57

33 a): Distribution of $|\cos \Theta|$ of muons with track segments in the $\mathrm{F} / \mathrm{B}$ muon chambers. The hatched histogram shows the muons in the toroidal region. $b$ ): Impact points in the $x-y$ plane of reconstructed muon tracks in the FI chambers on one door. . . . . . . . . . . . . . . . . . . . .

34 Corrected residuals for hits in the outer wires and inner wires for 8 point fits in FM and FO X and W layers. The Full Widths at Half Maximum (FWHM) of the distributions are shown in the plots. A detailed discussion of the resolution is given in paragraph $8.3 \ldots \ldots \ldots \ldots \ldots$

35 Momentum spread of reconstructed muons in $e^{+} e^{-} \rightarrow \mu^{+} \mu^{-}(\gamma)$ relative to the beam energy. Plotted is the quantity $E_{\text {beam }} /\left(q \cdot p_{\text {muon }}\right)$, where $E_{\text {beam }}$ is the LEP beam energy, $q$ is the reconstructed muon charge, and $p_{\text {muon }}$ the reconstructed muon momentum, in the S-region (a) and in the T-region (b). . . . . . . . . 59

36 Muon momentum resolution obtained in the $\mathrm{F} / \mathrm{B}$ muon system as a function of the cosine of the polar angle $\Theta$ of the track, for a muon momentum of $45 \mathrm{GeV}$. . 60

37 Time resolution . . . . . . . . . . . . . . . . . . . 60

38 RPCs time distribution, without subtracting the inter-bunchlet time, versus the RPCs identified bunchlet number. . . . . . . . . . . . . . . . 


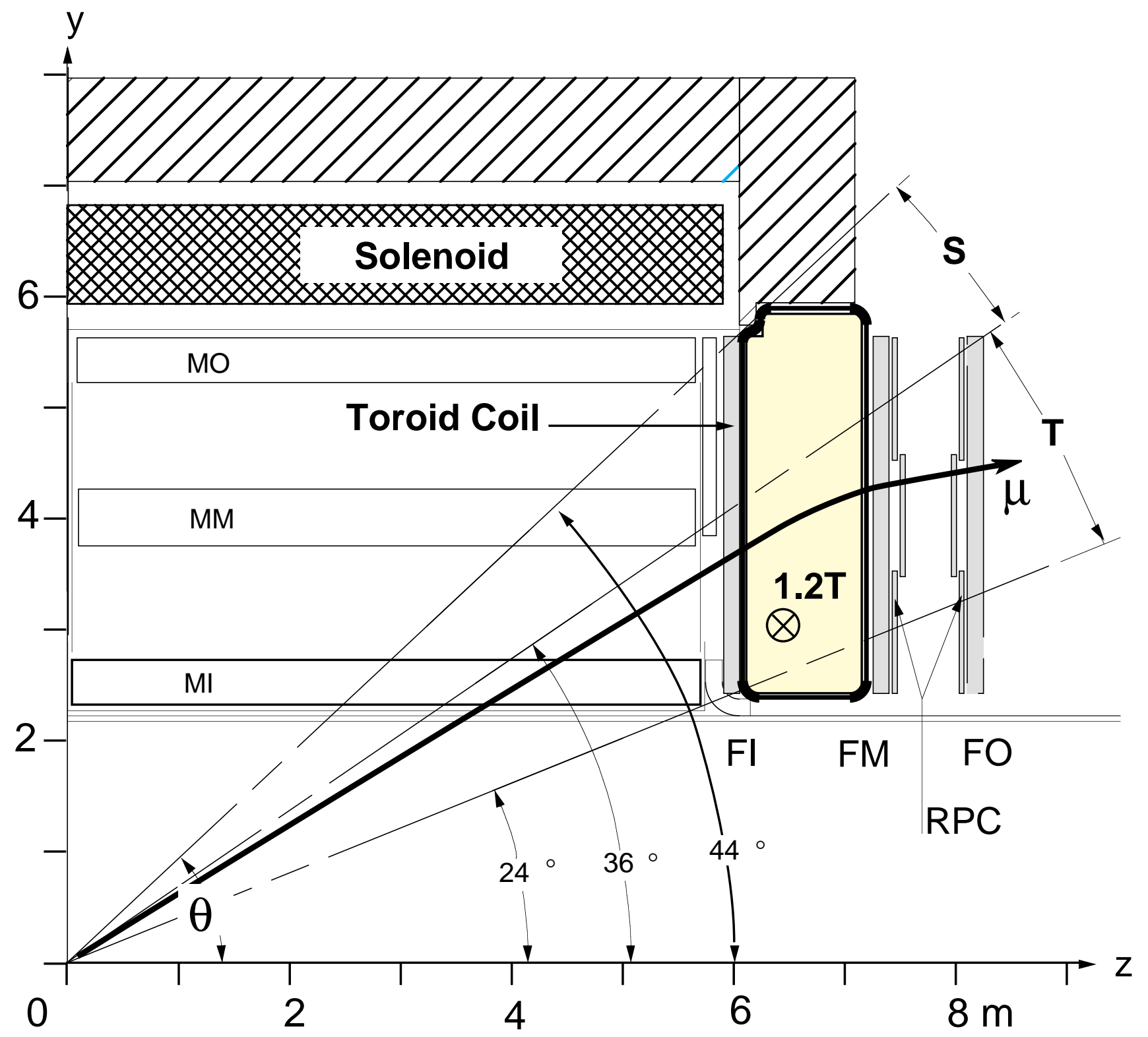

Figure 1: Side view of the F/B detector of L3 showing inner (FI), middle (FM), and outer (FO) drift chambers and RPCs for triggering. The two angular regions, $\mathrm{S}$ and $\mathrm{T}$, are indicated. 


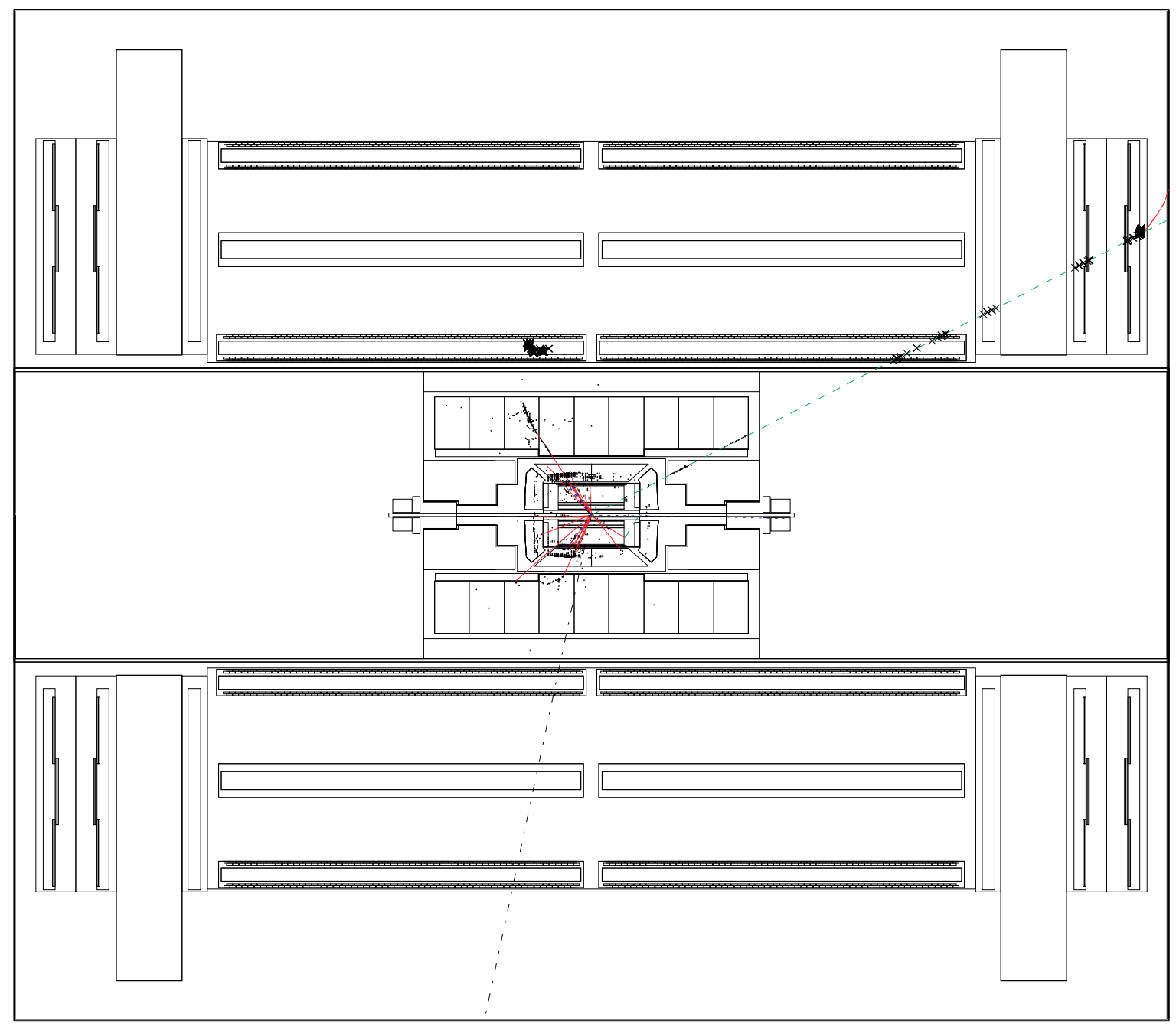

Figure 2: Simulated $\mathbf{e}^{+} \mathbf{e}^{-} \rightarrow \mathrm{W}^{+} \mathrm{W}^{-} \rightarrow \mu \nu_{\mu}+$ two jets event in the L3 detector. The direction of the undetected $\nu_{\mu}$ is indicated as well. The forward detector is essential for muon detection. 


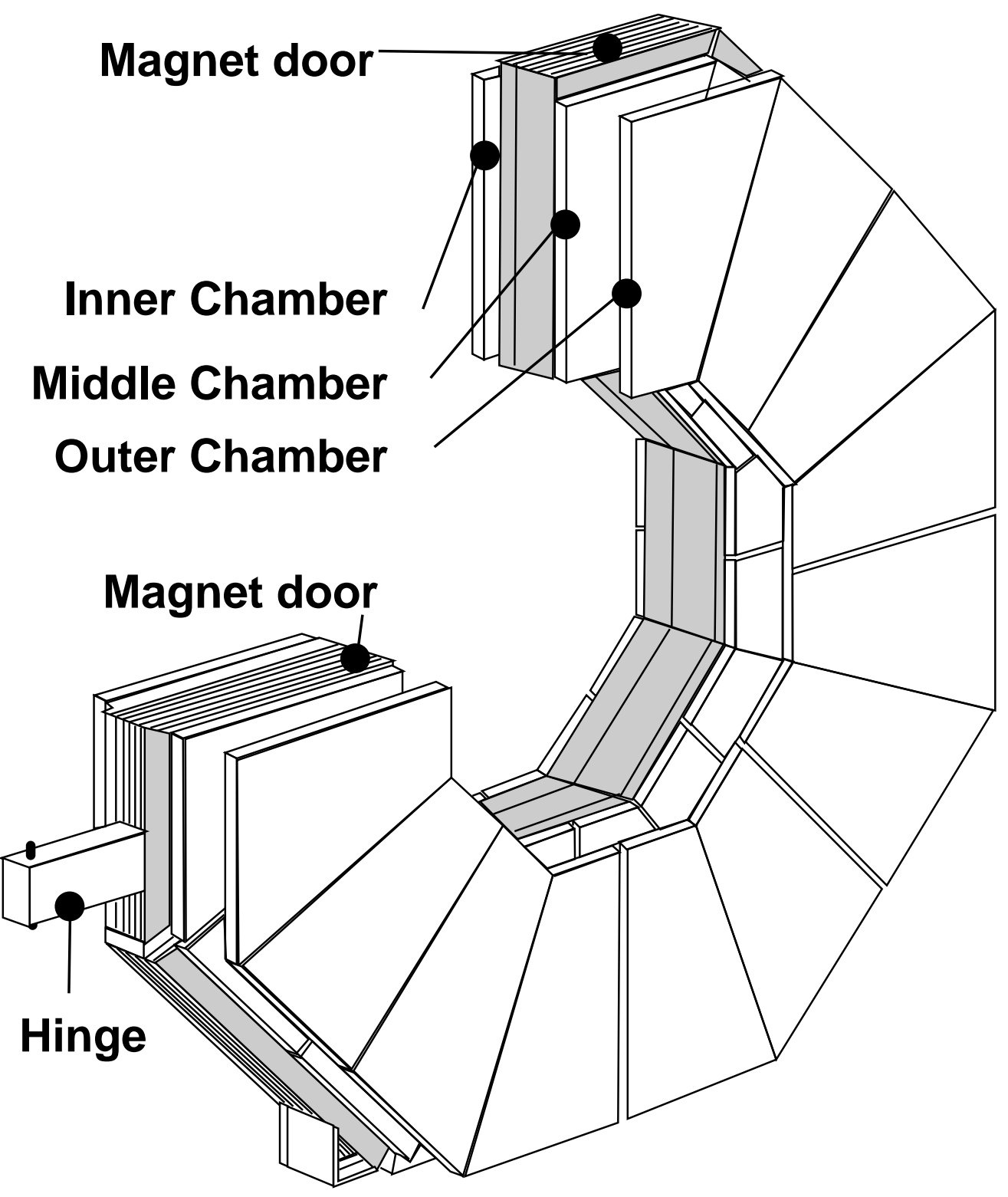

Figure 3: Schematic view of the L3 F/B muon detector. 


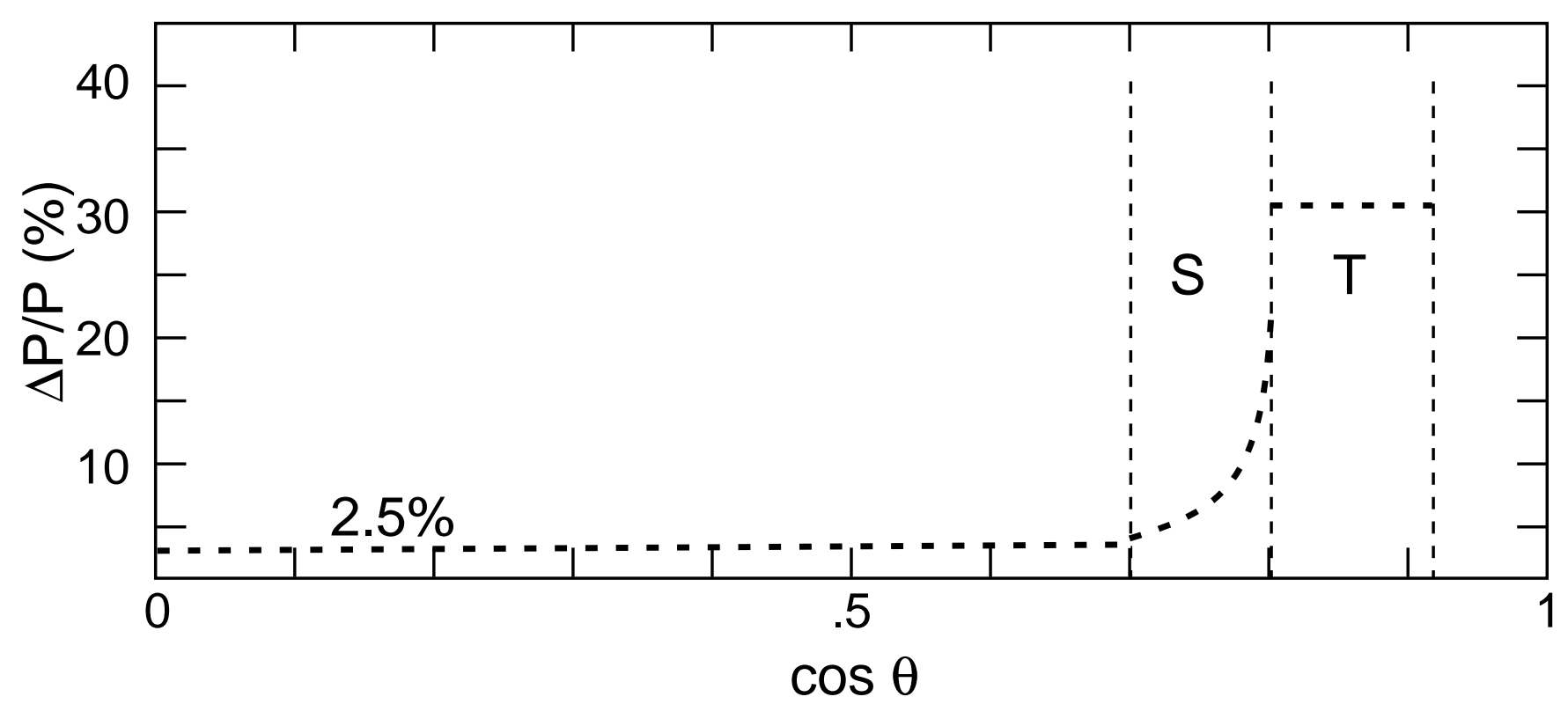

Figure 4: Expected muon momentum resolution as a function of polar angle. 


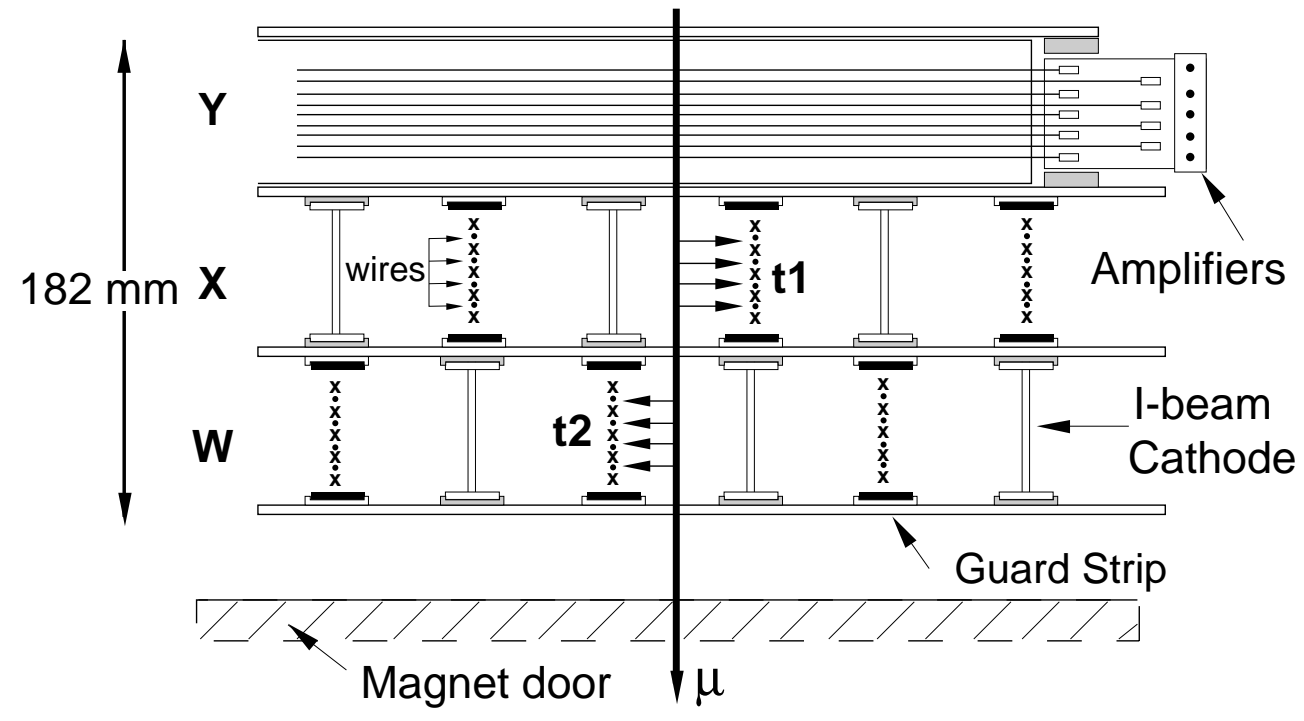

Figure 5: Drift chamber of 3 layers made of cells with 4 signal wires interspersed with 5 field wires and guard strips above and below the wire plane.

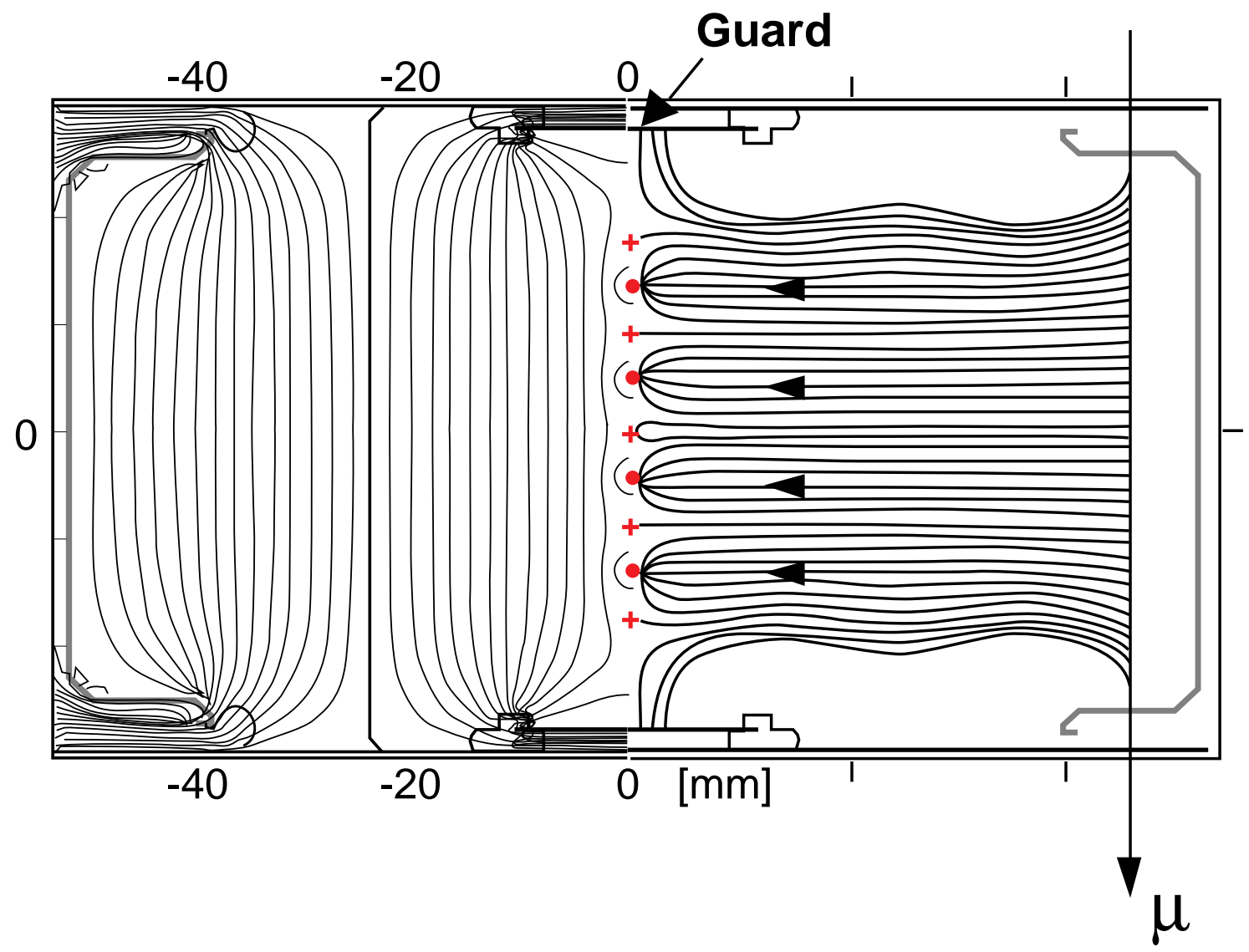

Figure 6: On the left, the equipotential configuration of a drift cell with 4 signal wires is shown. On the right, drift field trajectories are shown. 


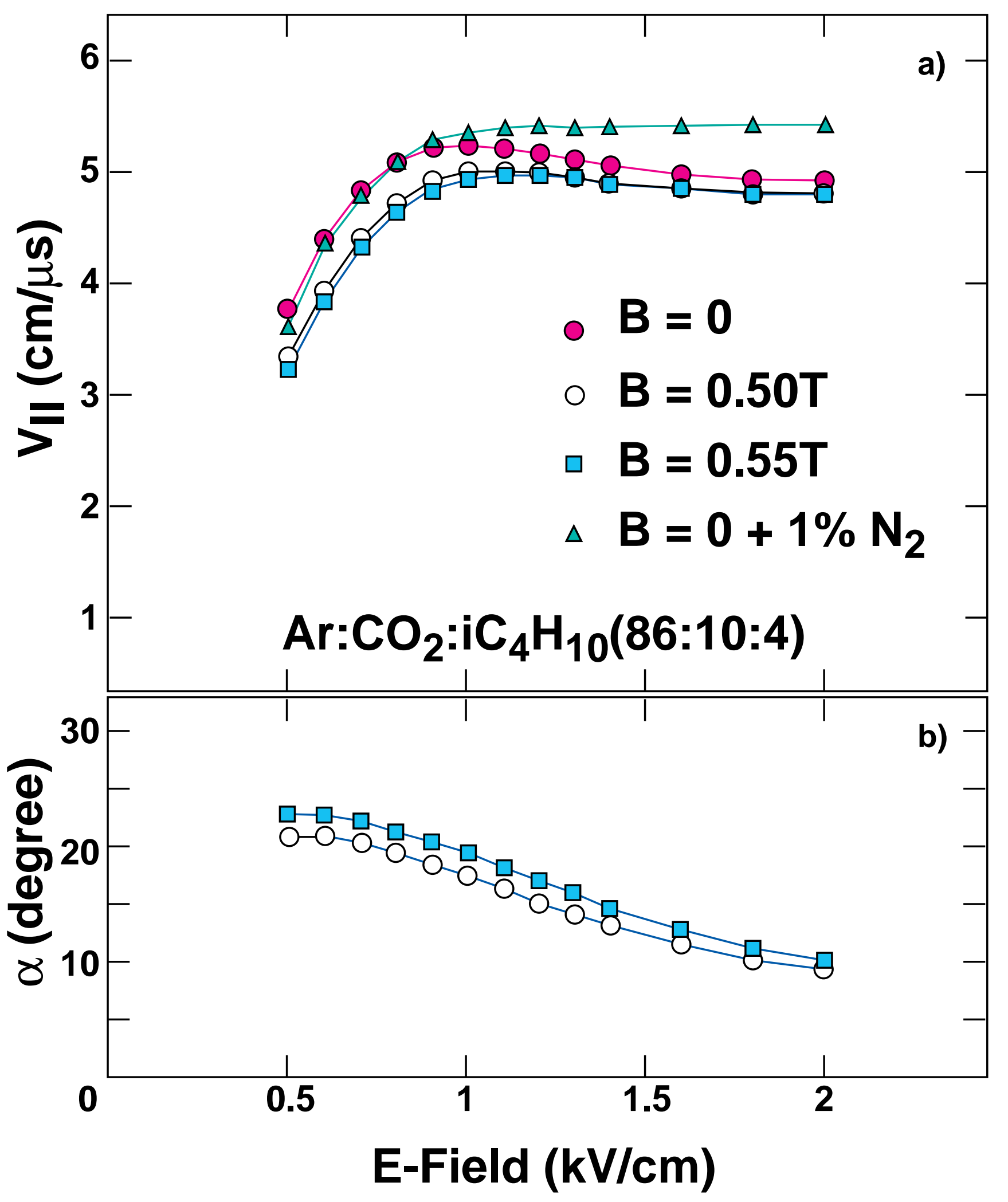

Figure 7: a) Drift velocity along the electrical field for different $B$ field values. The upper curve shows, for $\mathrm{B}=0$, the effect of a $1 \% N_{2}$ contamination. b) Lorentz deflections as a function of $\mathrm{E}$ for $\mathrm{B}$ field values of $0.5 \mathrm{~T}$ and $0.55 \mathrm{~T}$. 


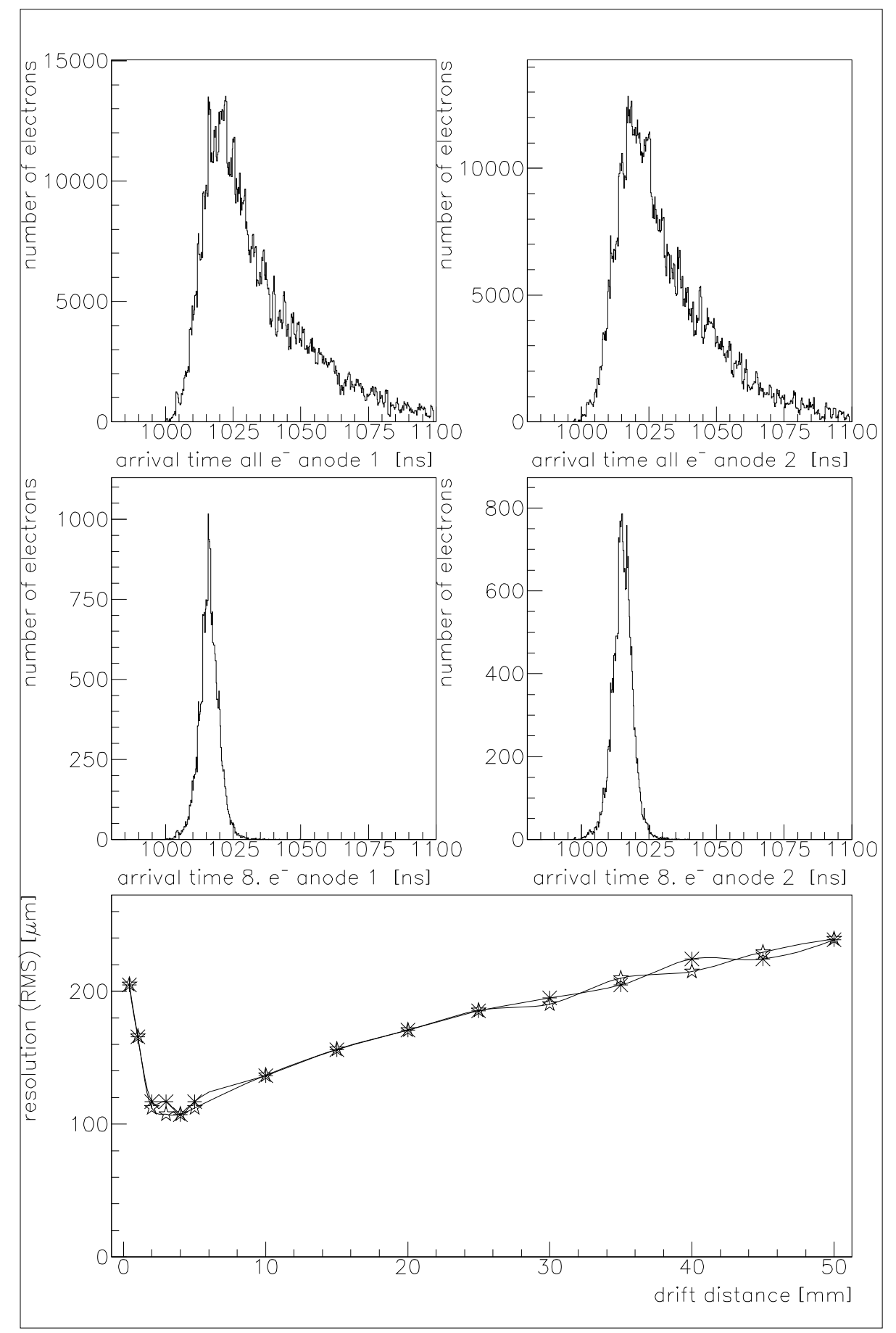

Figure 8: Monte Carlo simulation of: a) Spectrum of arrival times for two wires (on the left an outer, on the right an inner wire) for a $26 \mathrm{~mm}$ drift distance. b) Jitter in the arrival of the $8^{\text {th }}$ electron. c) Resolution expected from the time jitter for various drift distances. 


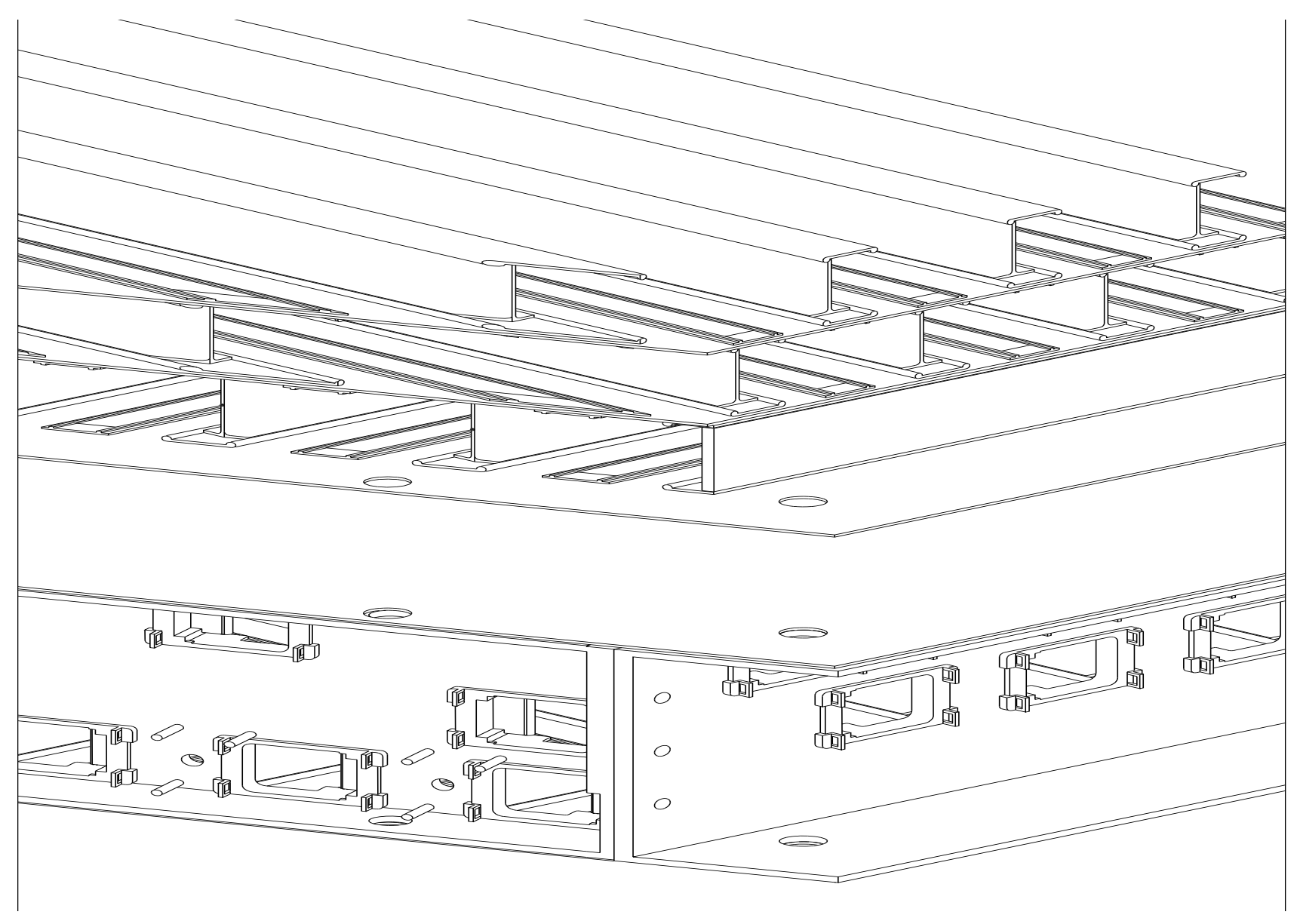

Figure 9: View of a chamber. The upper part gives the interior structure of the W, X and Y layers. The lower part depicts the corresponding chamber frame with the holes for the wire planes. 


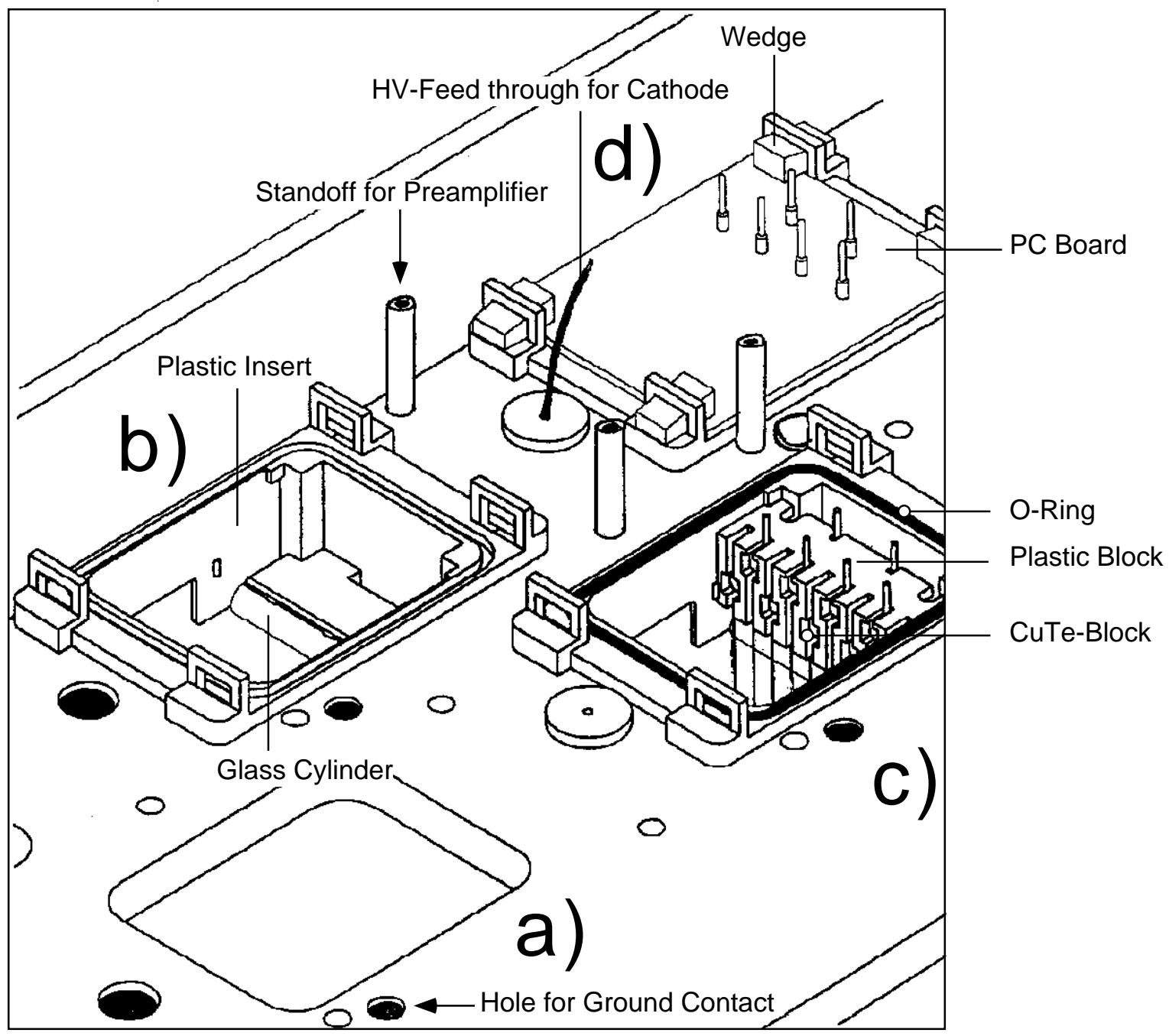

Figure 10: Detailed sketch of an endframe showing a) holes where plastic inserts will be placed, b) plastic insert already in place showing the glass cylinder, c) one which has wires on $\mathrm{Cu}-\mathrm{Te}$ blocks, and d) one cell gas sealed and ready for electrical connections. 

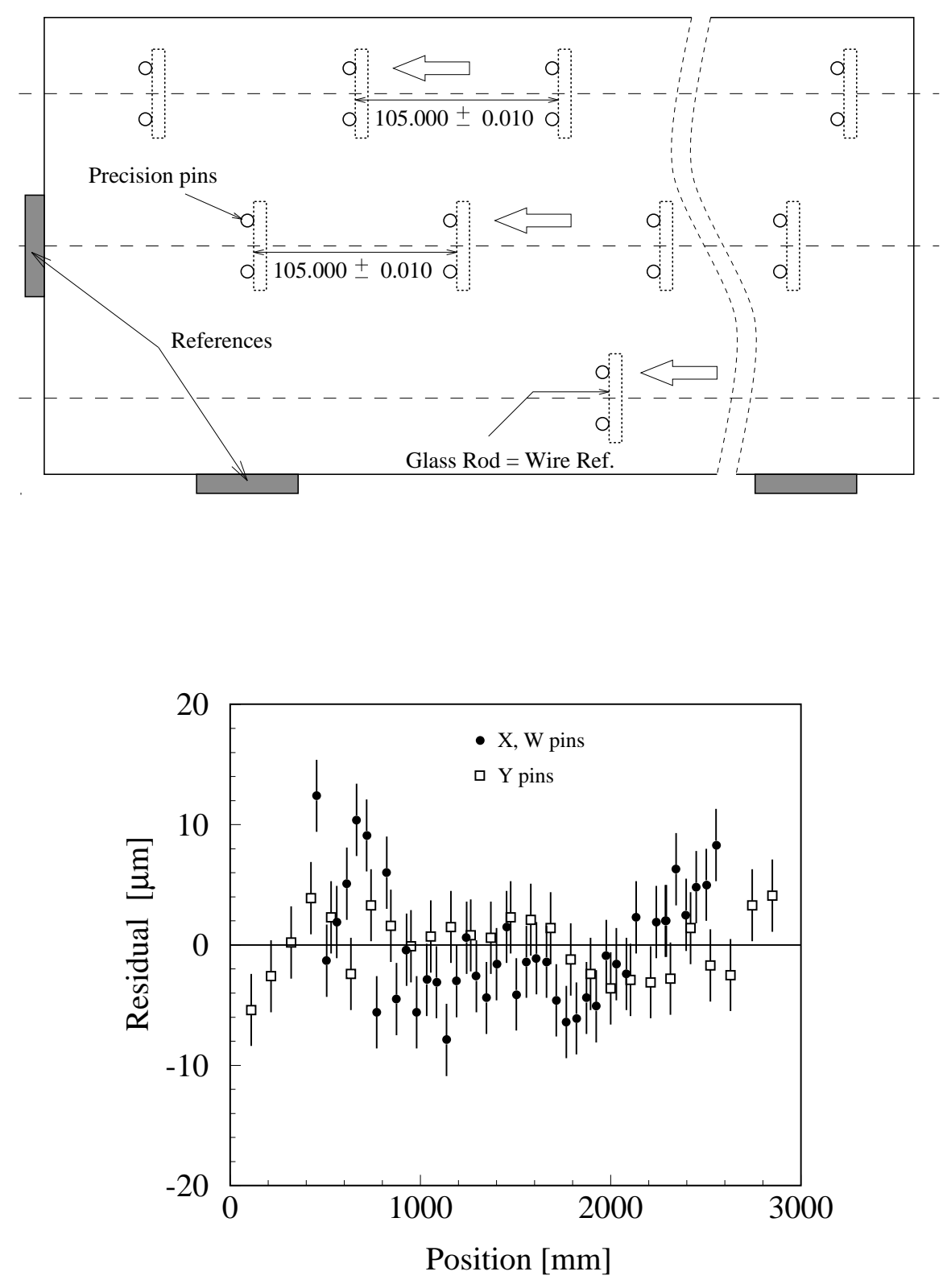

Figure 11: a) Sketch of the precision template used to position glass cylinders which define wire plane locations. b) Residuals showing template accuracy. 


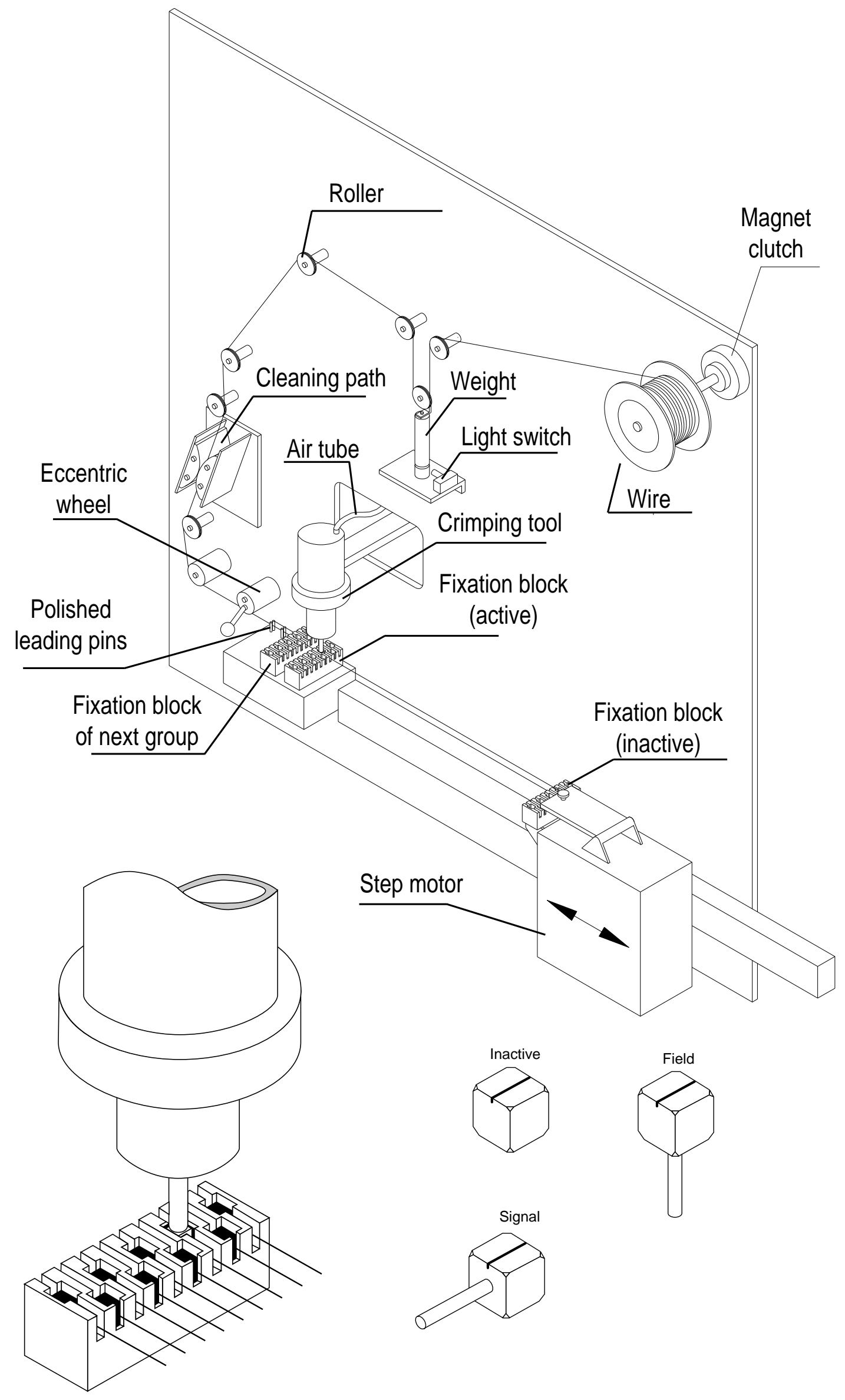

Figure 12: A schematic drawing of the wiring machine (only one of the nine wires shown), with closeups of the crimping tool with fixation block and different types of $\mathrm{Cu}-\mathrm{Te}$ cubes. 

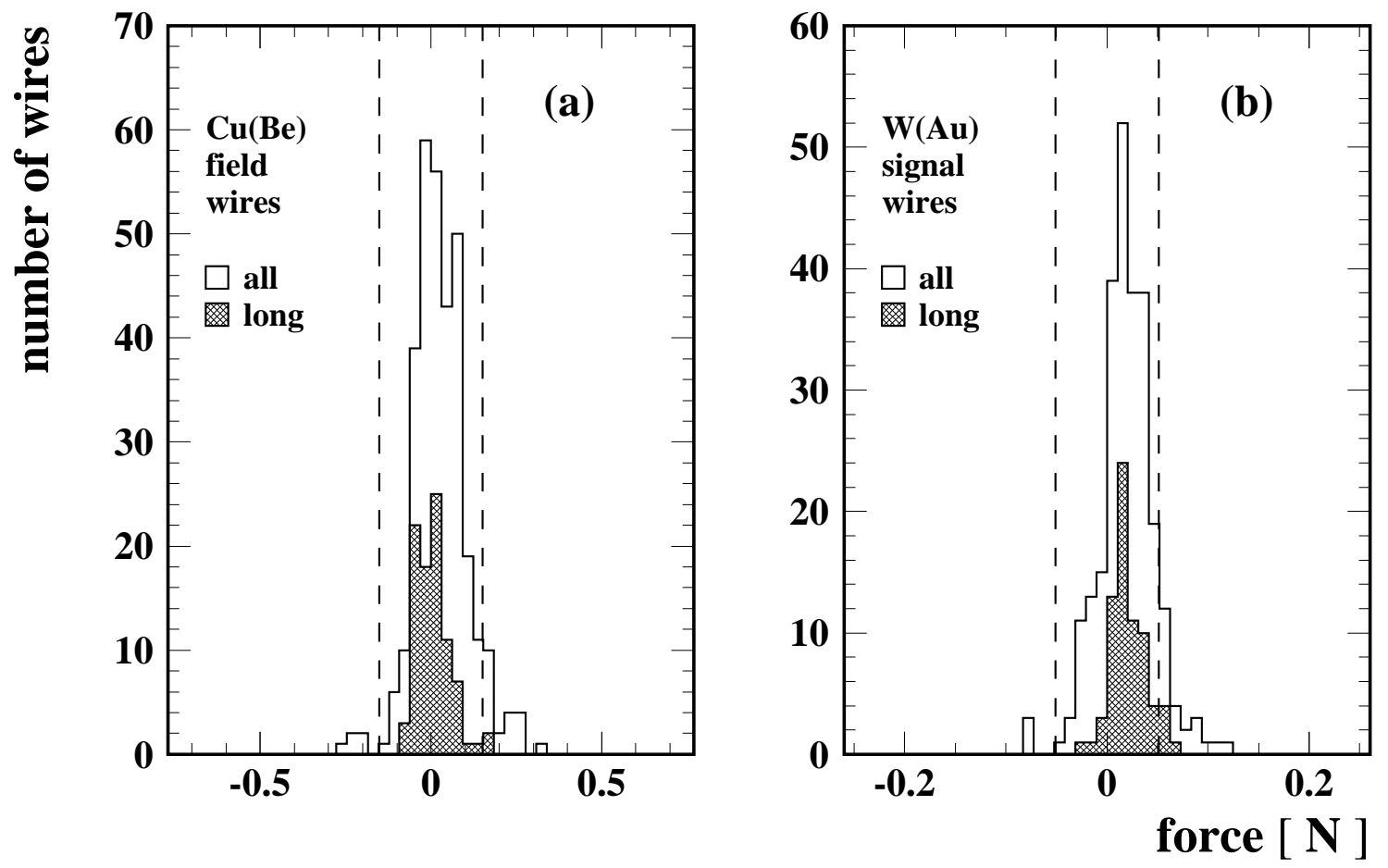

Figure 13: Mechanical tension deviations from the design values for (a) field and (b) signal wires. Shadowed are the critical long wires. The dashed lines indicate the design tolerance of $\pm 4 \%$. 

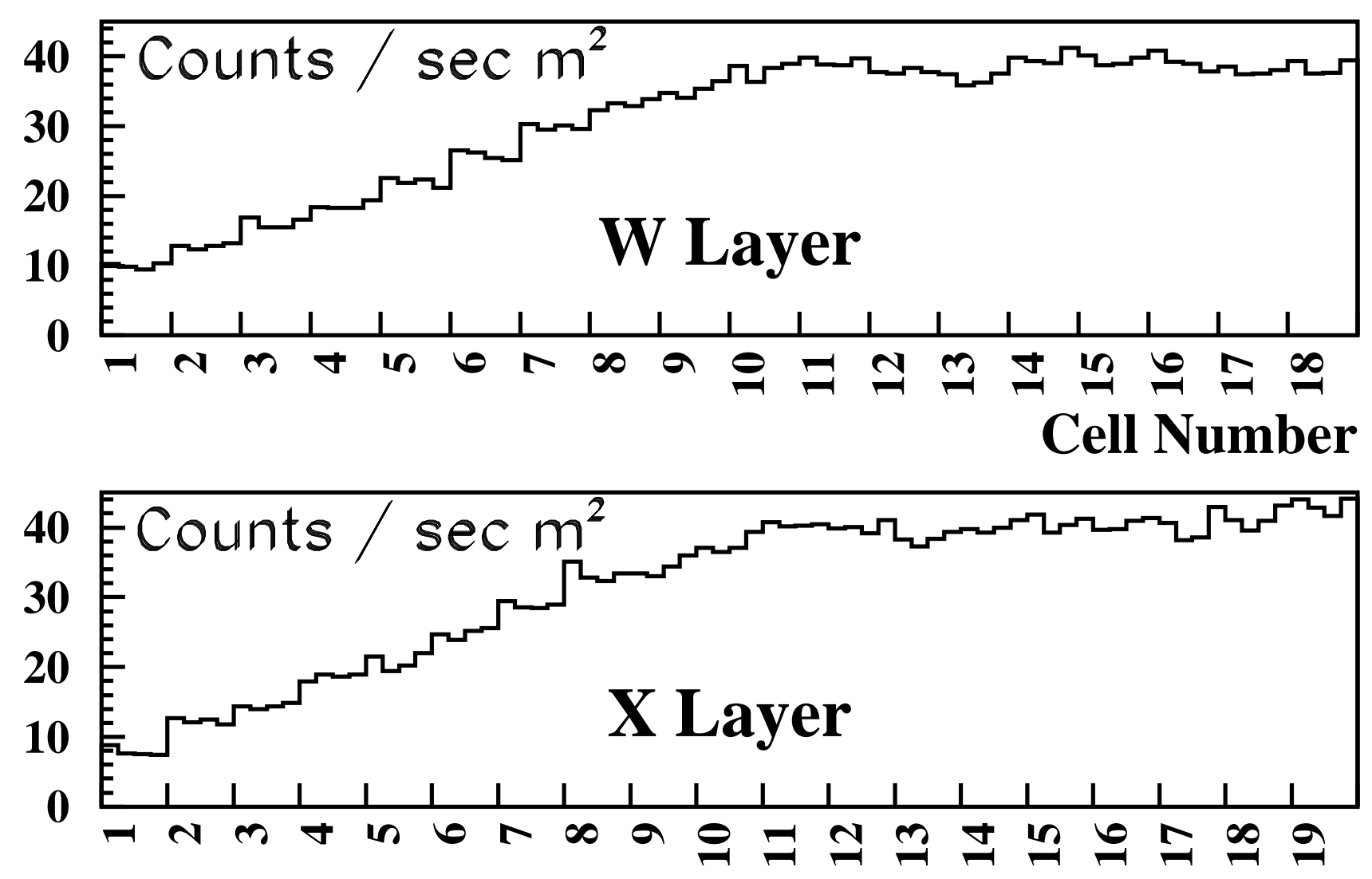

Cell Number

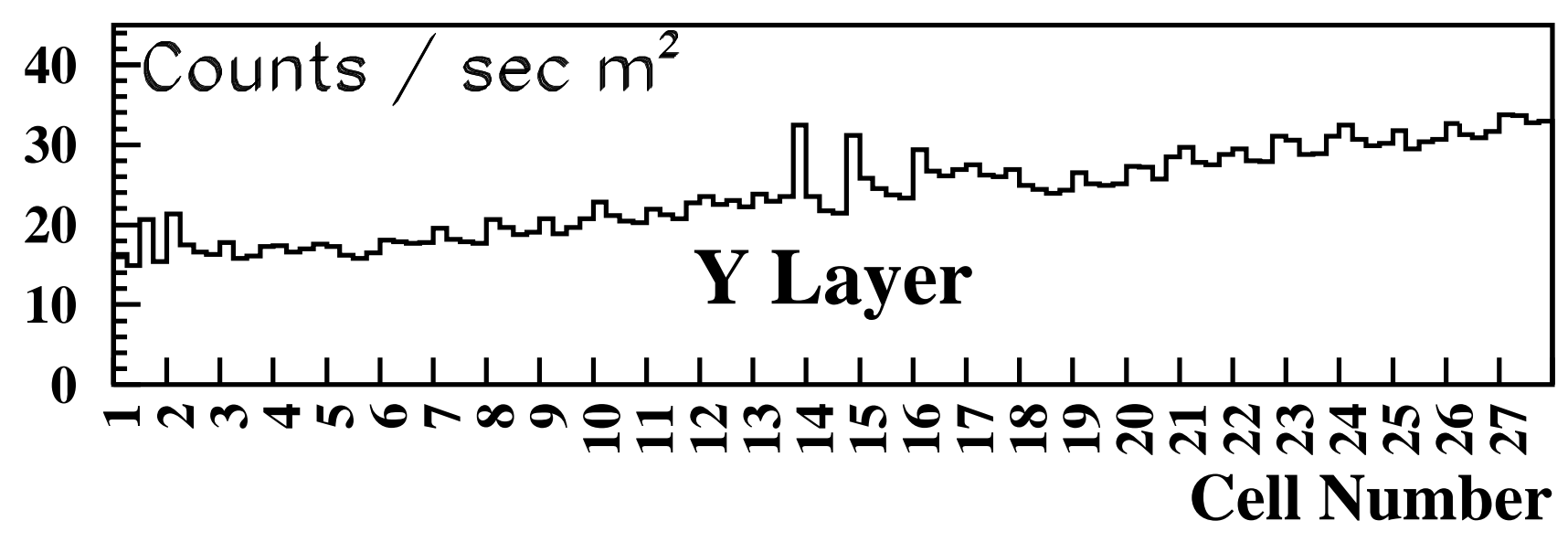

Figure 14: Typical counting rates using cosmics in one FB chamber. Rates are proportional to wire lengths, which explains the pattern observed in the $\mathrm{Y}$ layer (slow increase from cell number 1 to 27 ) and in the $\mathrm{W}$ and $\mathrm{X}$ layers (increasing from cell number 1 to 11 and staying constant for the rest of the cells. 


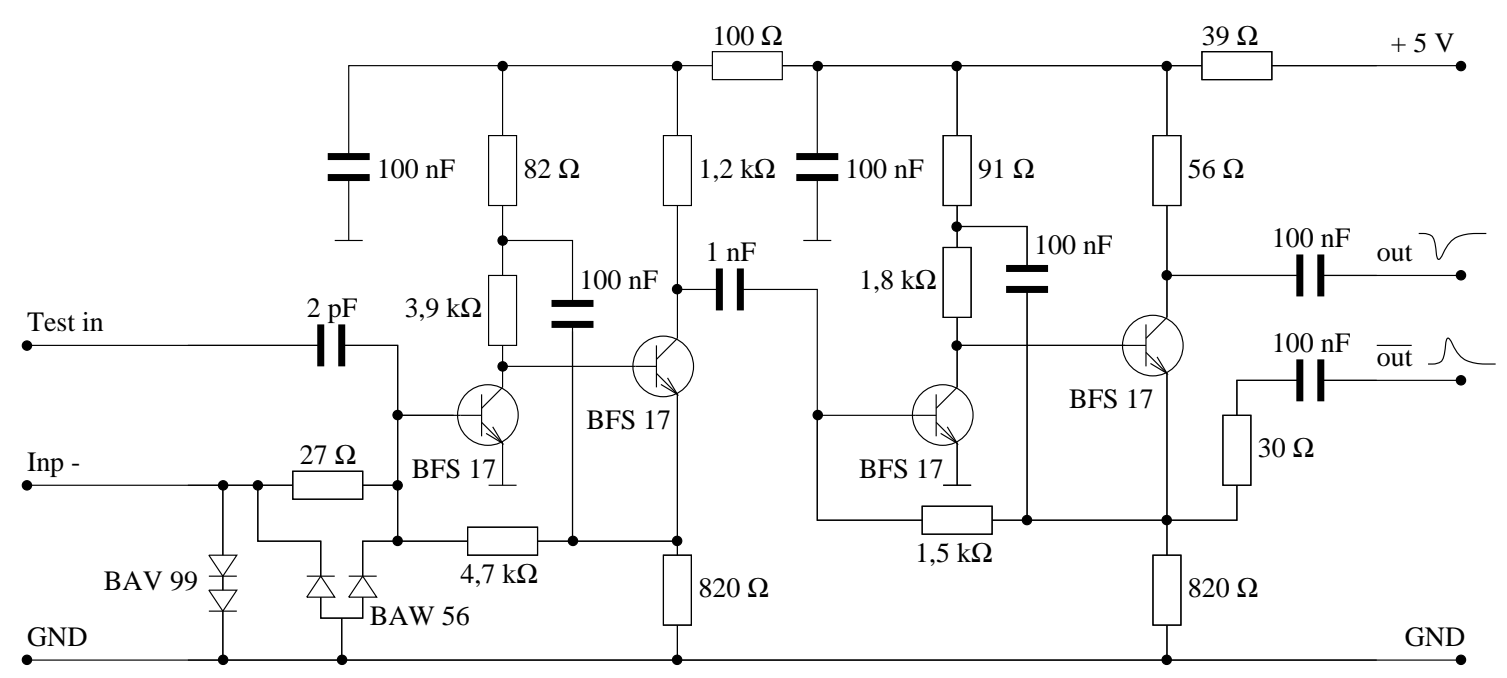

Figure 15: Preamplifier circuit. 


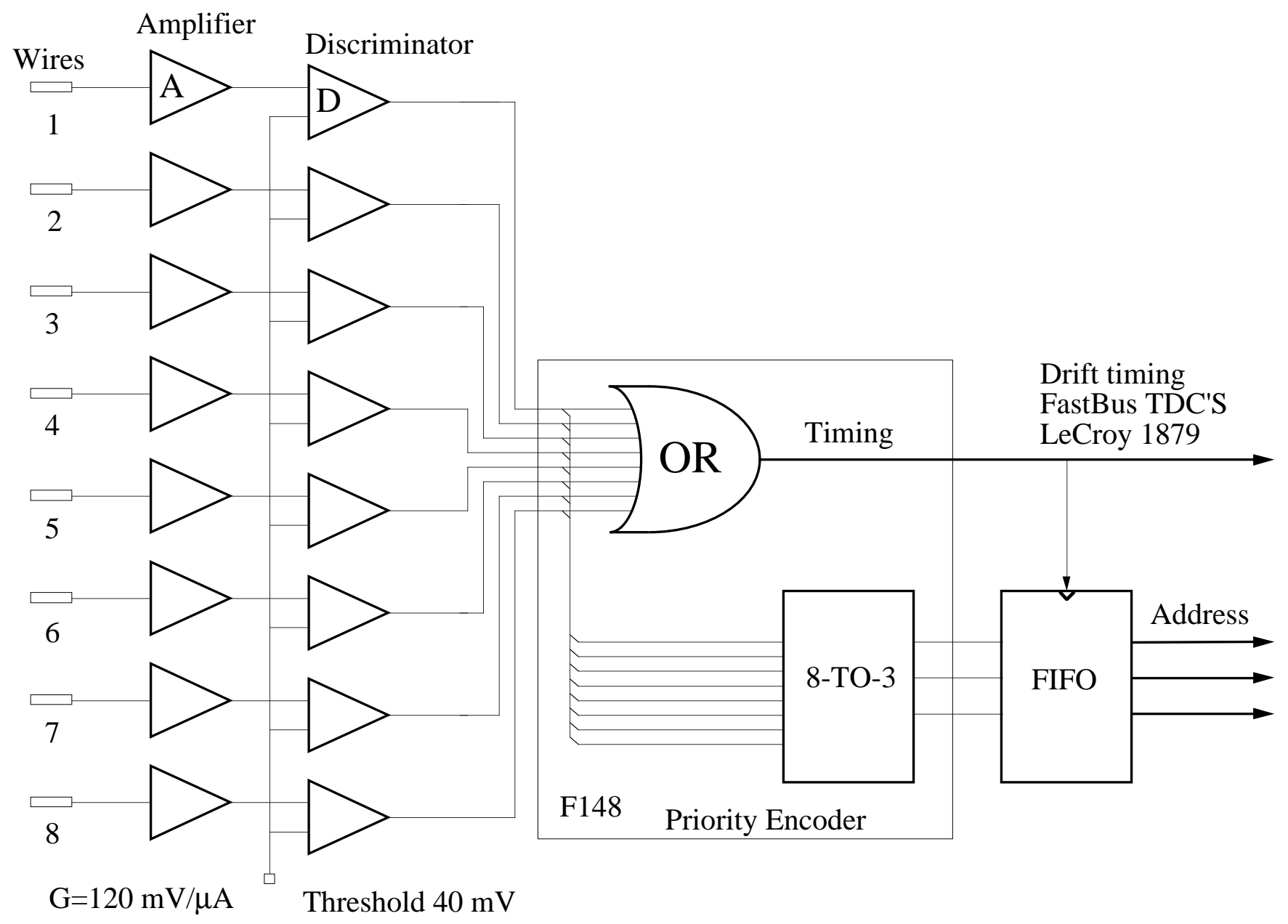

Figure 16: Multiplexer of 8:1 channels with address recording. 


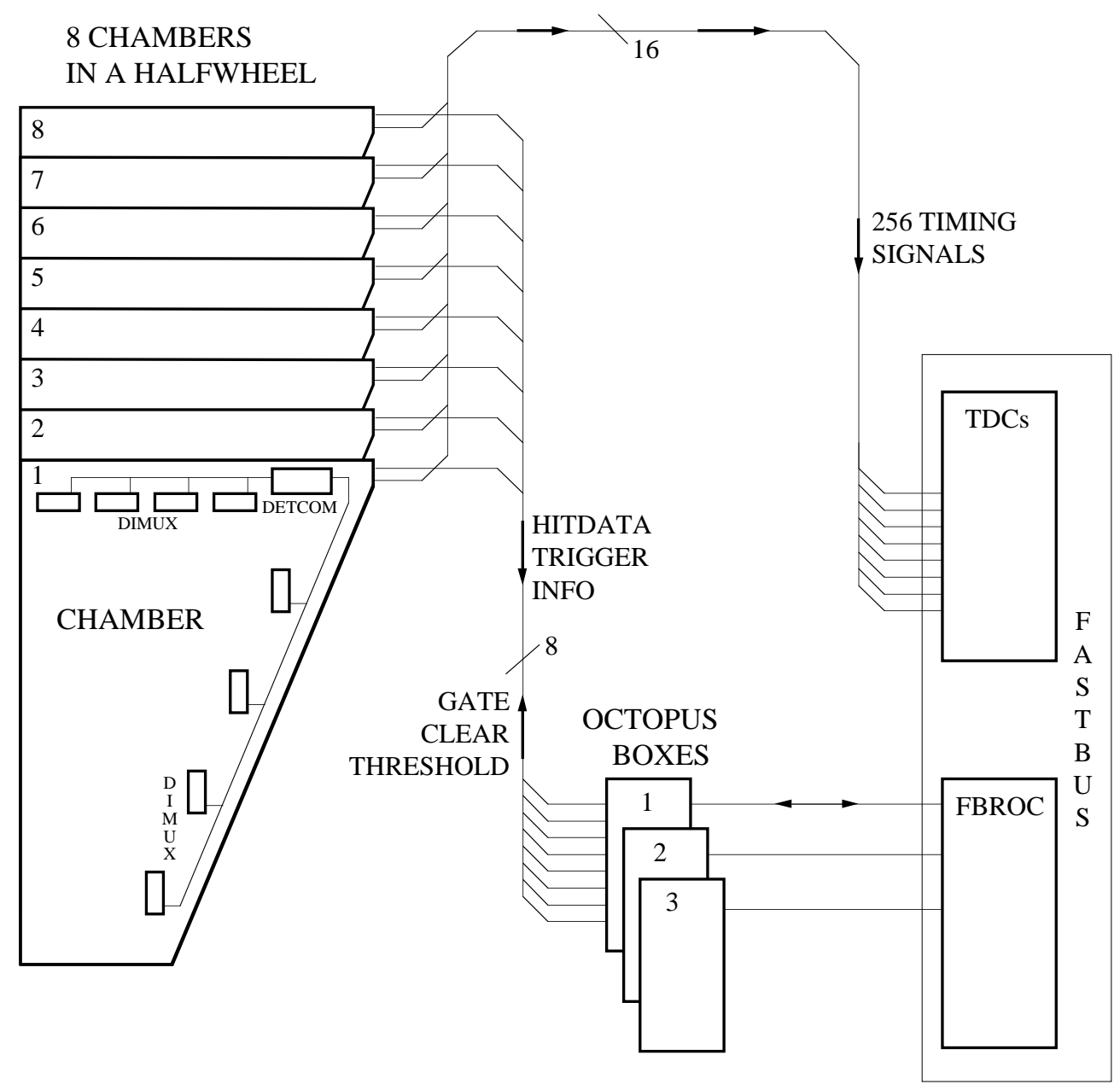

Figure 17: Readout schematic. 


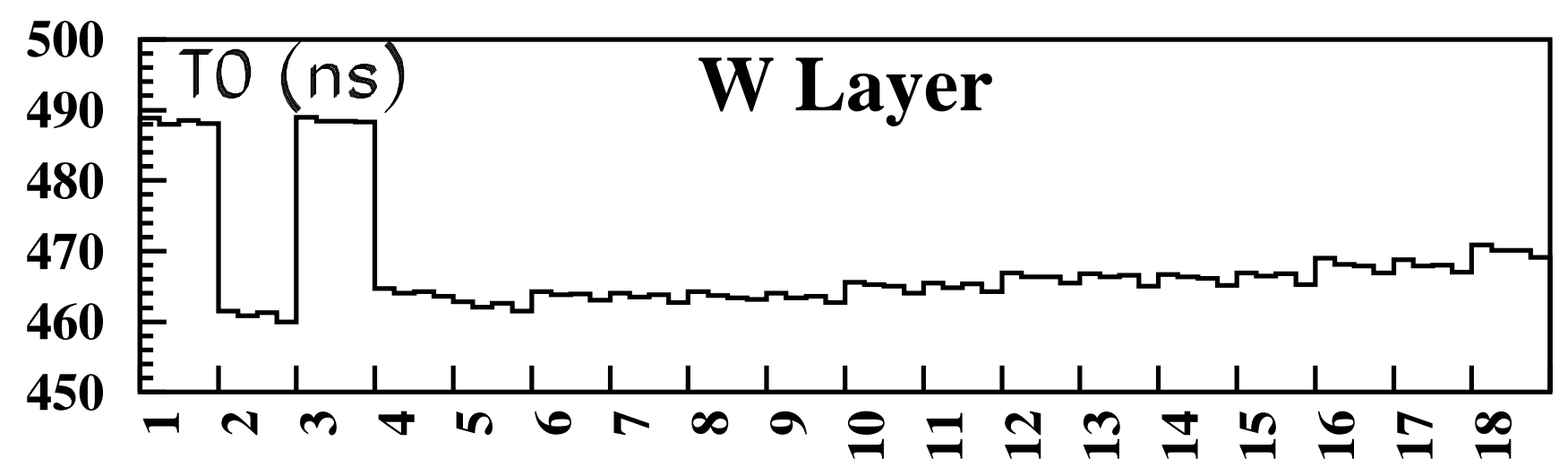

Cell Number
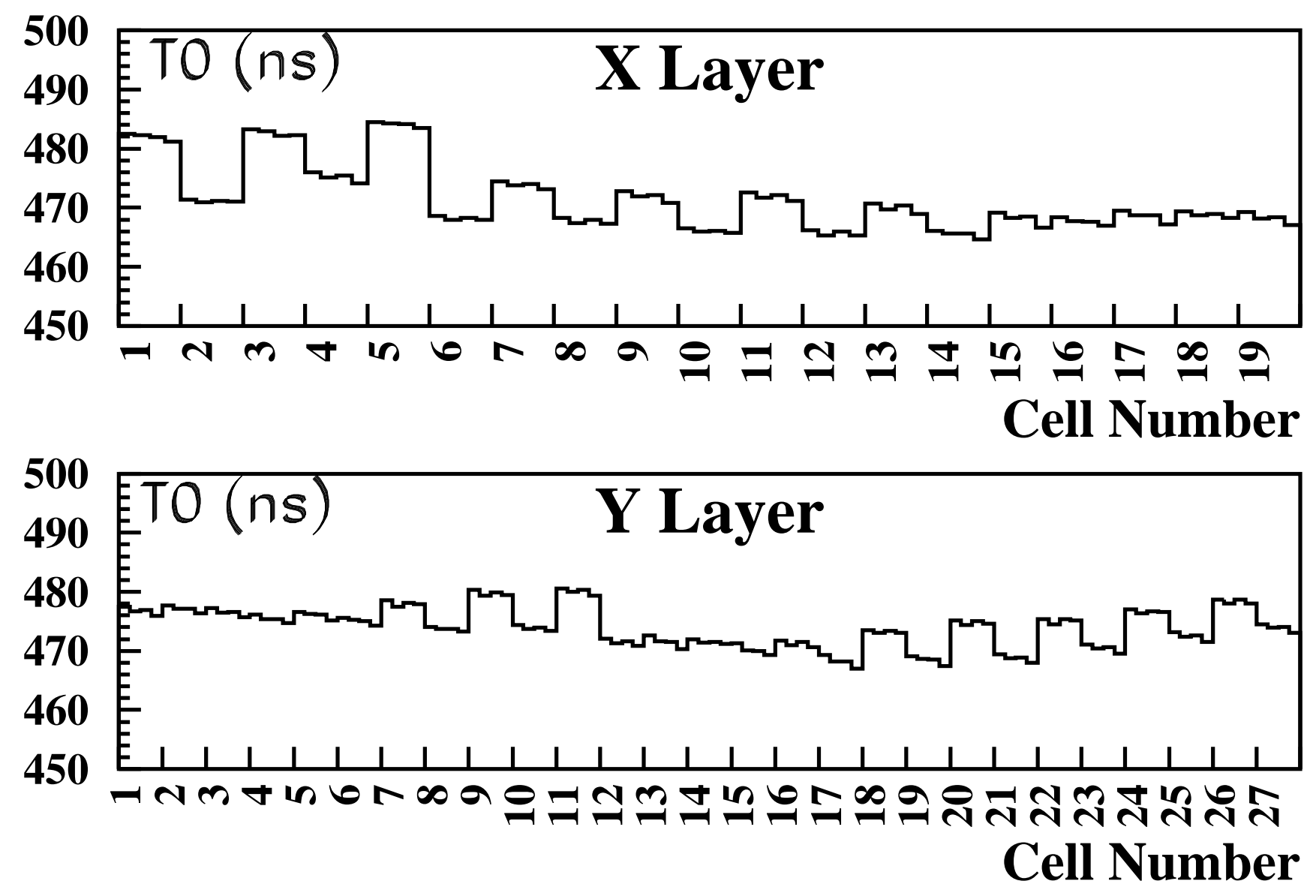

Figure 18: T0CAL test as performed to one of the chambers providing the relative channel to channel timing calibration for all wires in all layers. 


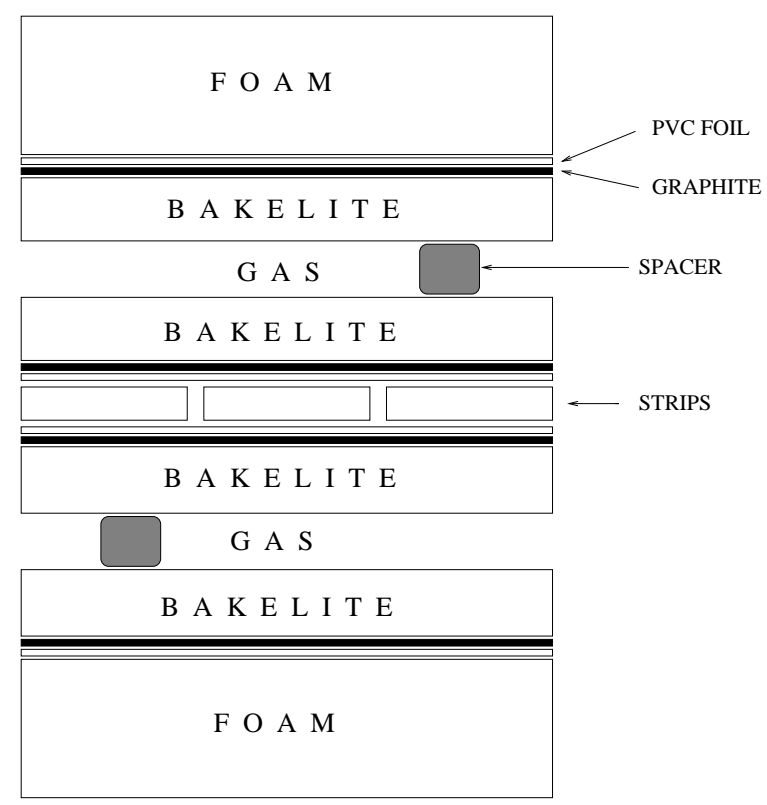

Figure 19: Cross section of a double-gap RPC (not to scale). 

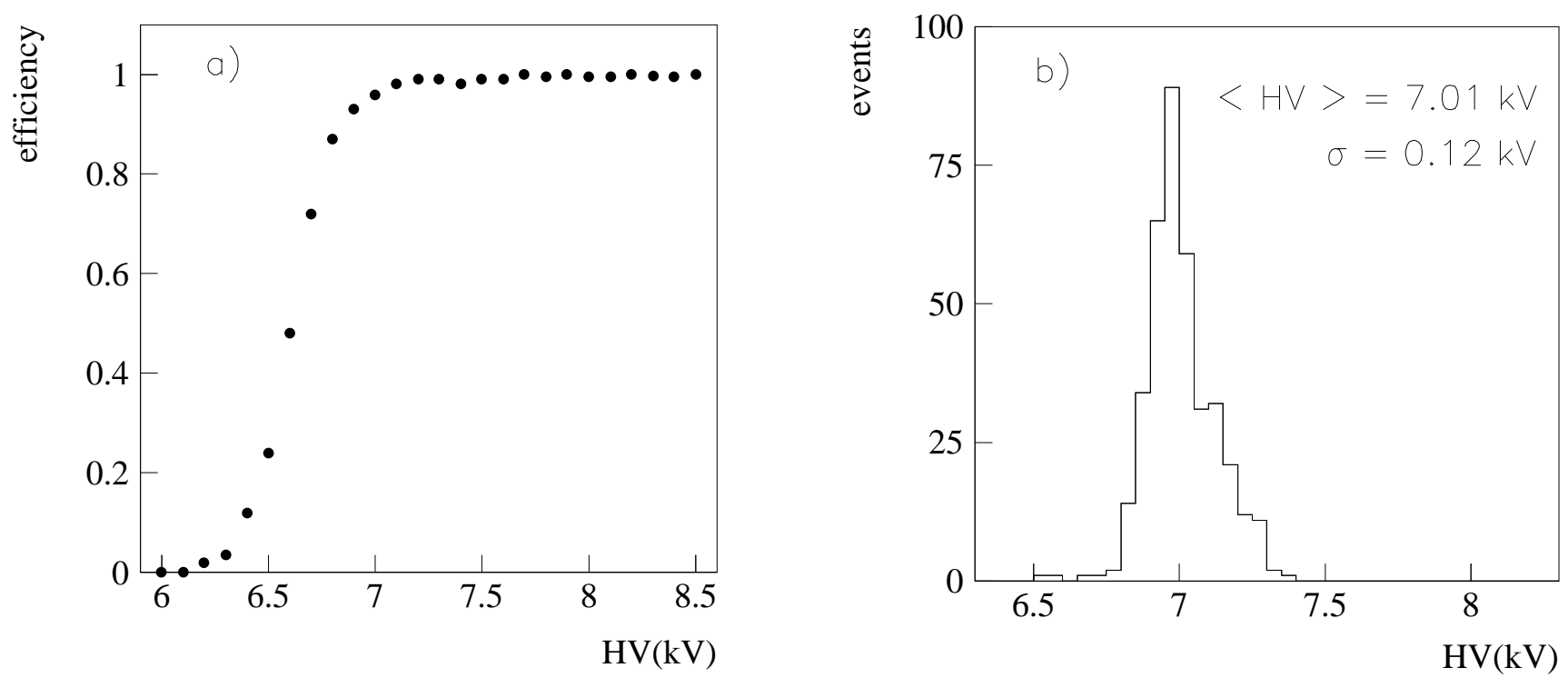

Figure 20: a) Typical plateau curve; b) Distribution of the $90 \%$ efficiency working point for all tested chambers.
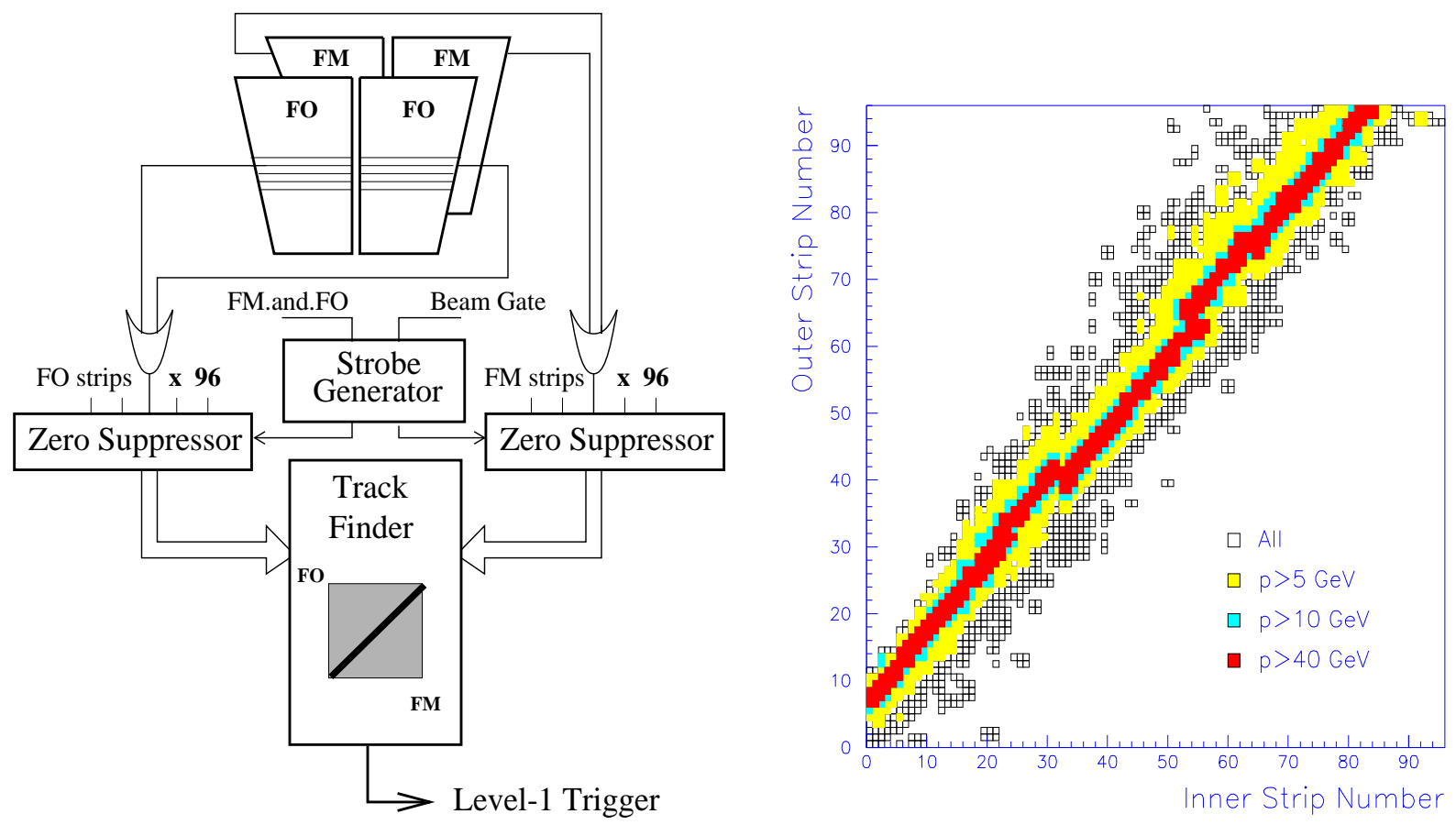

Figure 21: a) Trigger logic; b)Monte Carlo simulation of the Trigger Matrix for muons with different momenta. 


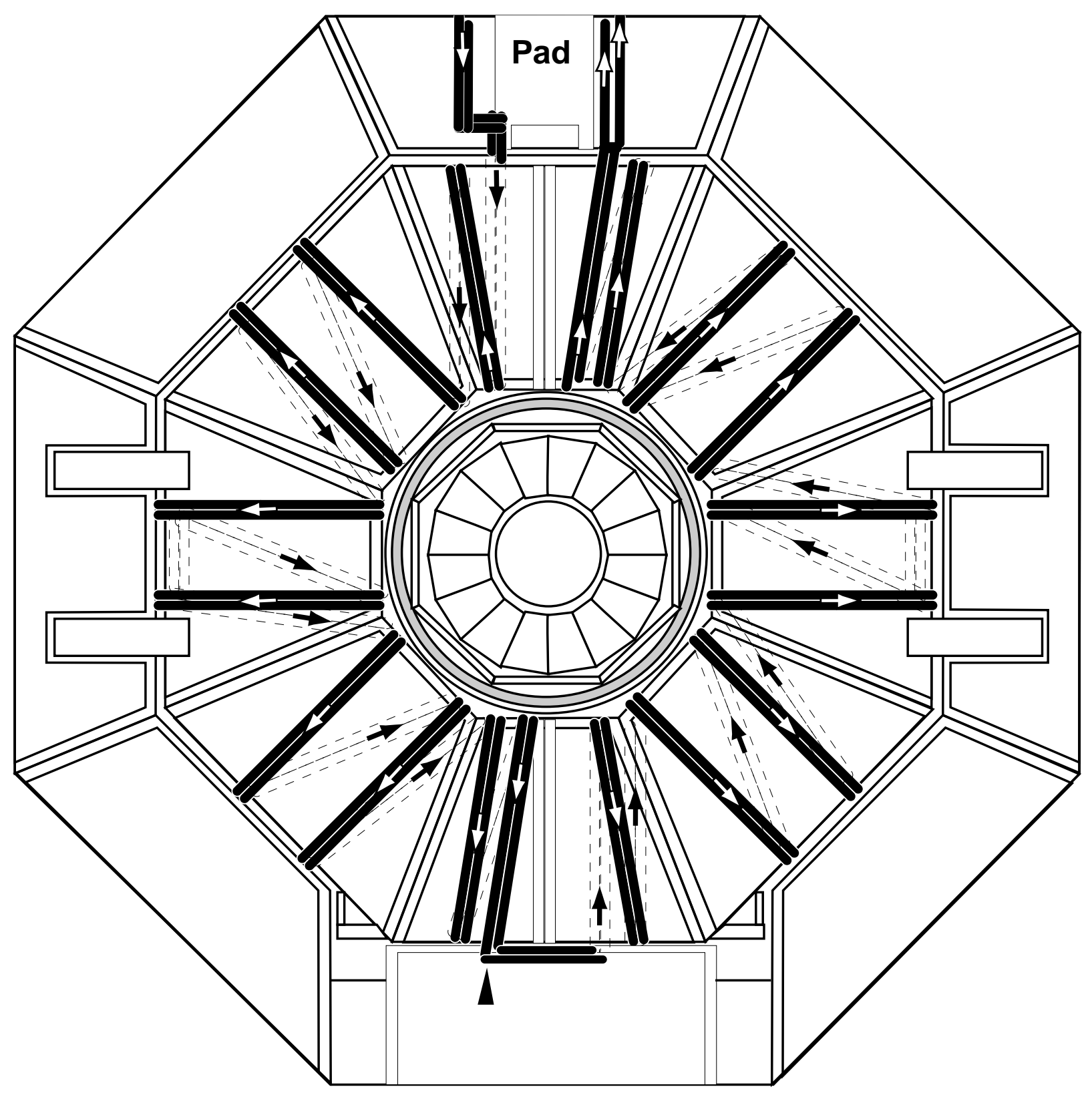

Figure 22: End view of the L3 magnet with the 36 turns of one toroidal coil. 


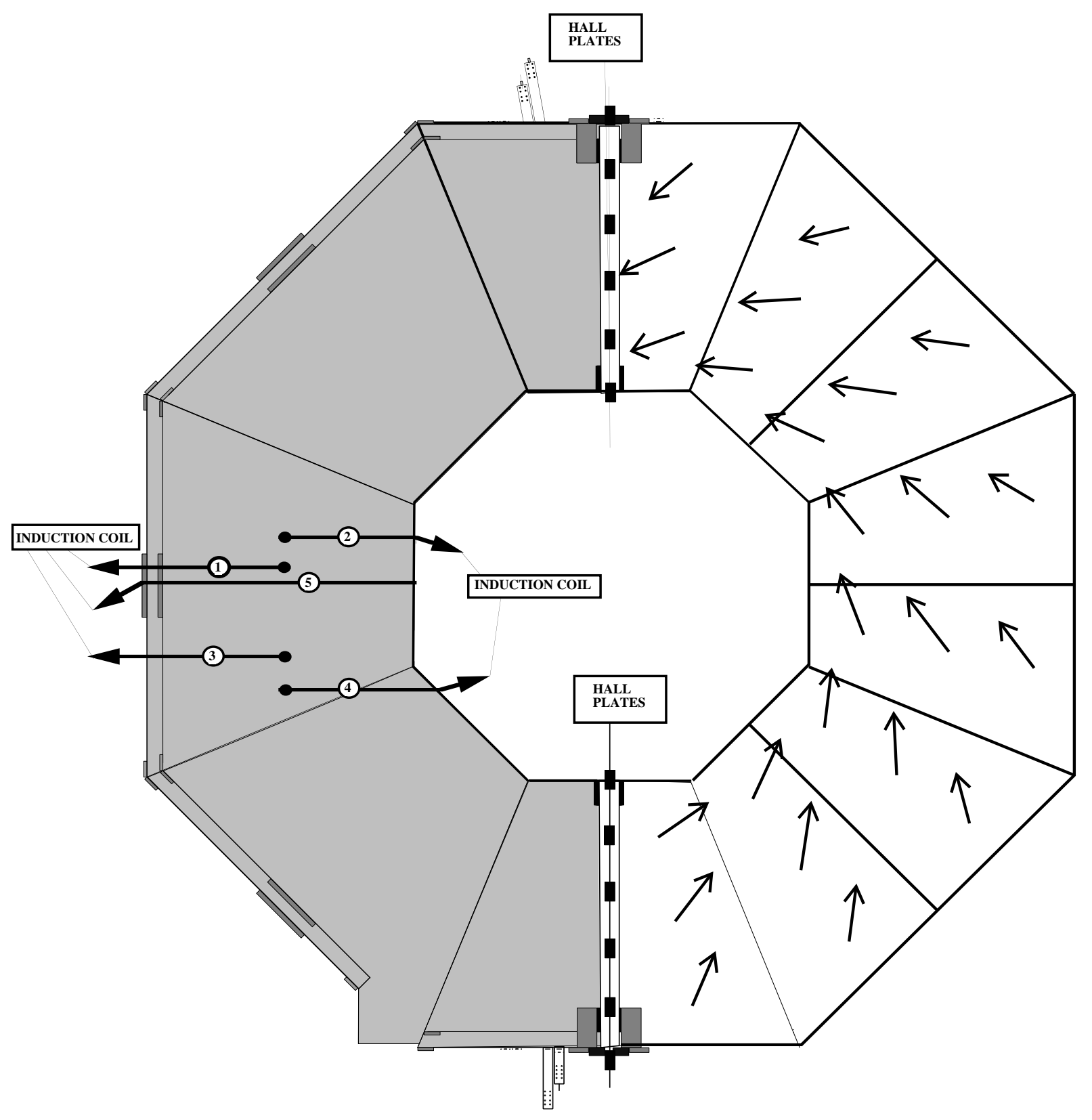

Figure 23: Left side: Location of field measuring devices. Right side: magnetic field vectors inside the iron according to a TOSCA calculation. 


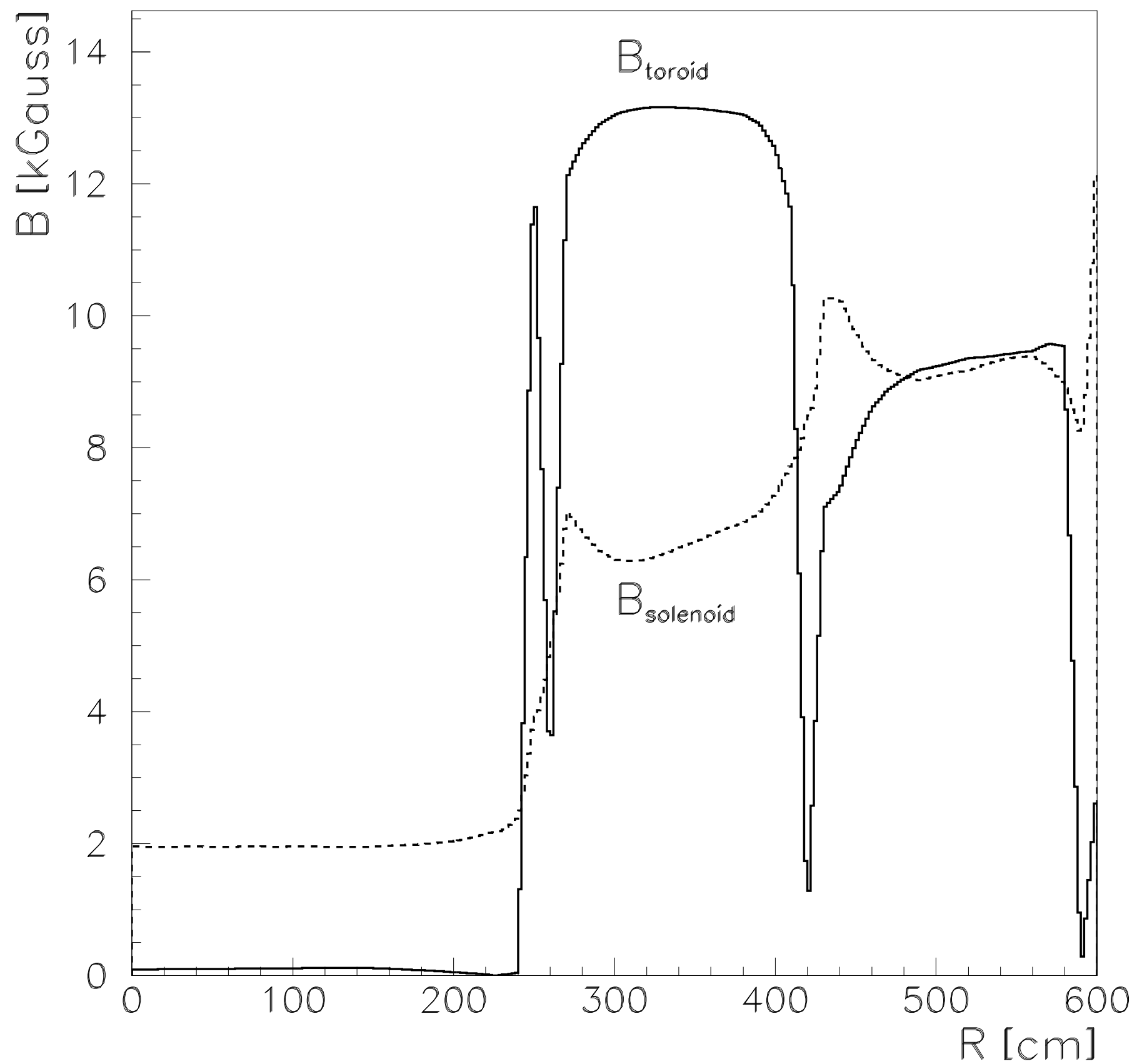

Figure 24: Calculated toroidal field strength as function of the distance to the beam axis $\mathrm{R}$ at $\Phi=0^{\circ}$, averaged over the thickness of the door which extends from 2.4 to $5.9 \mathrm{~m}$. The dips correspond to air gaps. The solenoidal part in the air and its return flux in the doors is shown as dotted curve. 


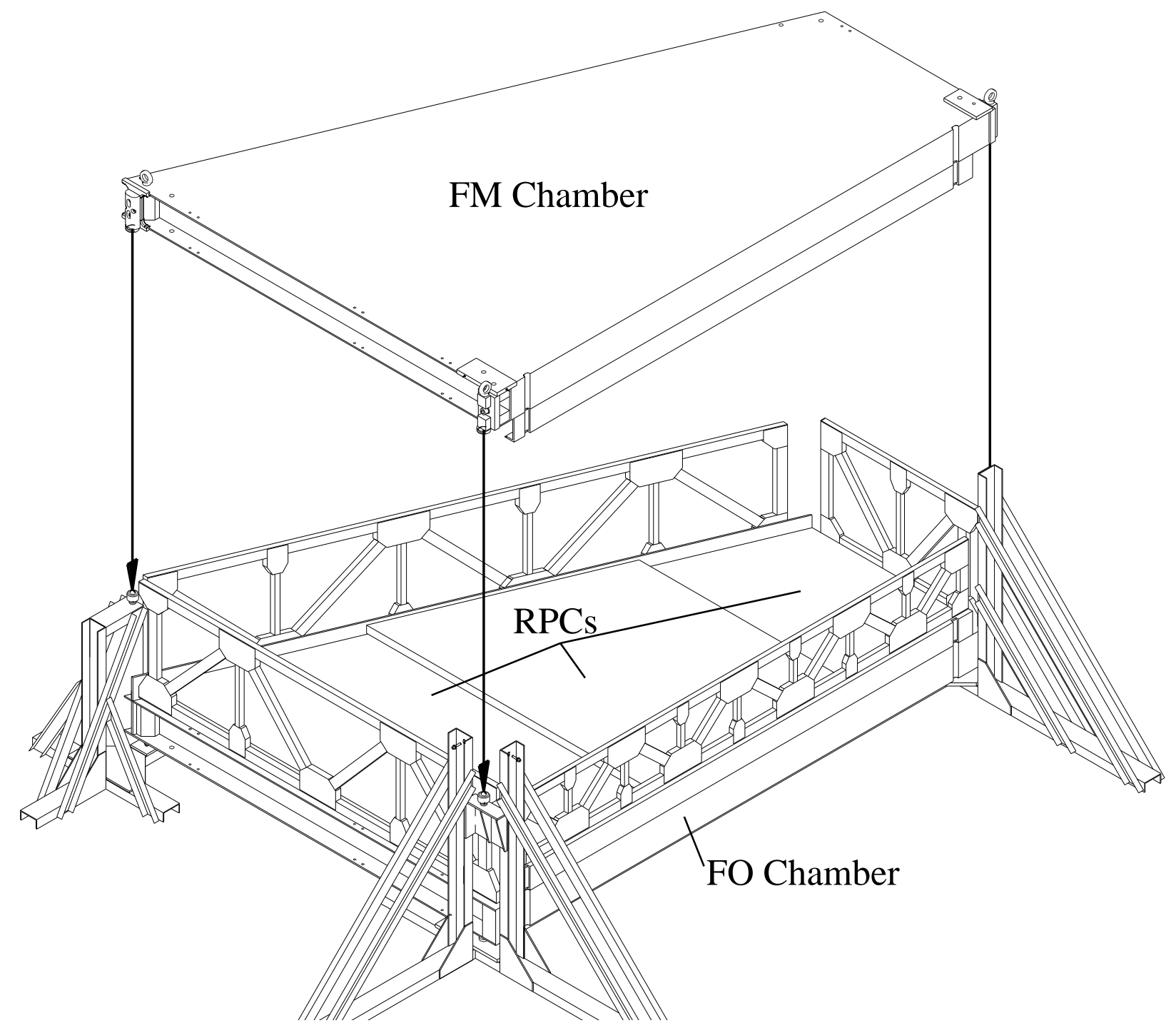

Figure 25: Schematic view of module assembly. 

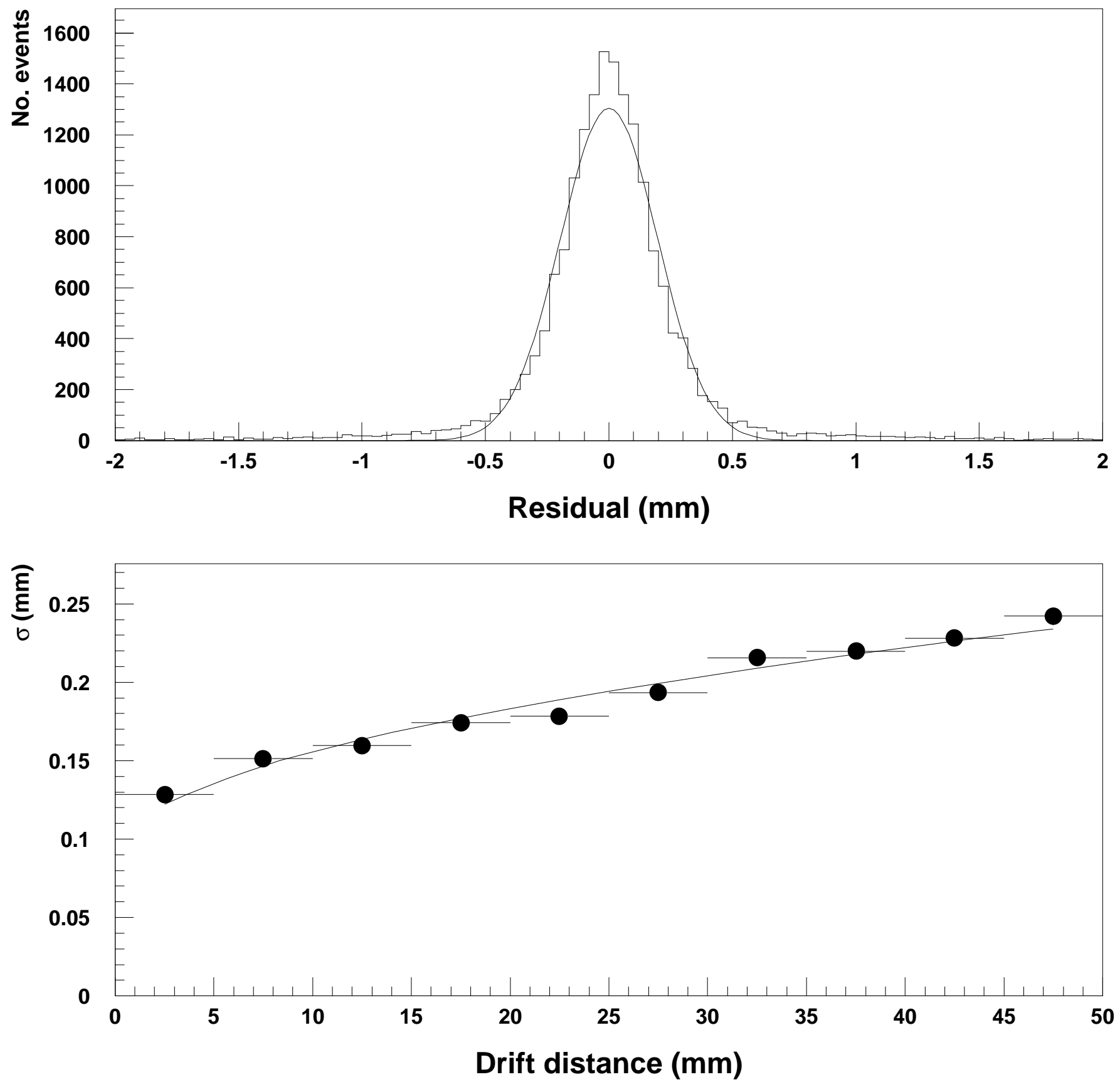

Figure 26: (a) Distribution of the fit residuals of the cosmic ray tracks. (all selected events, see text) The gaussian fit has a standard deviation of $200 \mu \mathrm{m}$. (b) Dependence of $\sigma$ on the drift distance. The curve is $\sigma=87+21 \times \sqrt{\text { distance }(\mathrm{mm})} \mu \mathrm{m}$. 


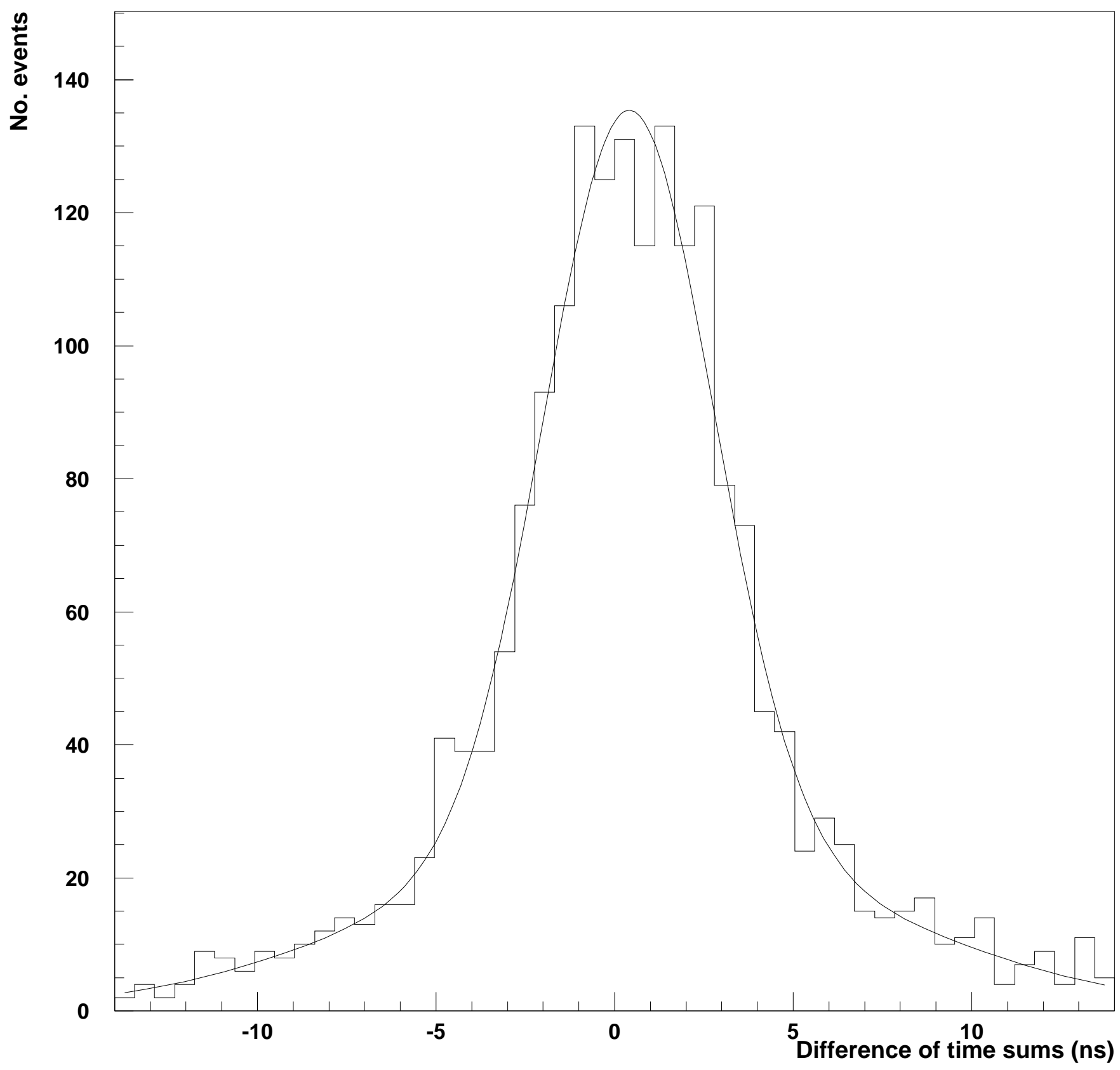

Figure 27: Distribution of the difference of the mean drift-time sums of the two chambers forming a module (see text). 

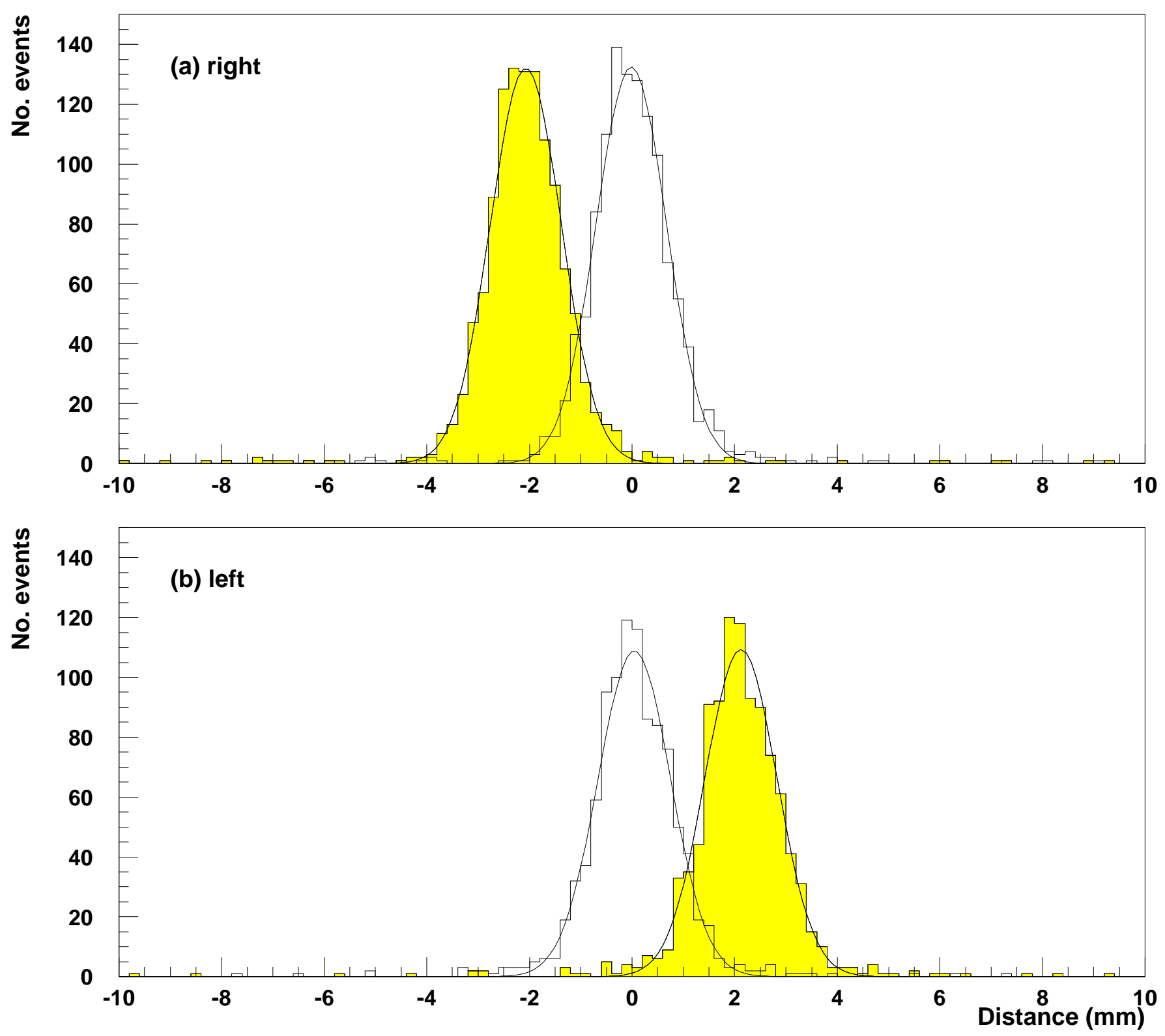

Figure 28: The distance between the two segments of a track fitted in the $\mathrm{X}$ and $\mathrm{W}$ layers measured at the midplane (solid histogram). Tracks on the right (a) and left (b) sides of the $\mathrm{X}$-wires are shown separately together with a gaussian fit. The shaded histogram is obtained when the same tracks are analyzed with a time offset of $20 \mathrm{~ns}$. 


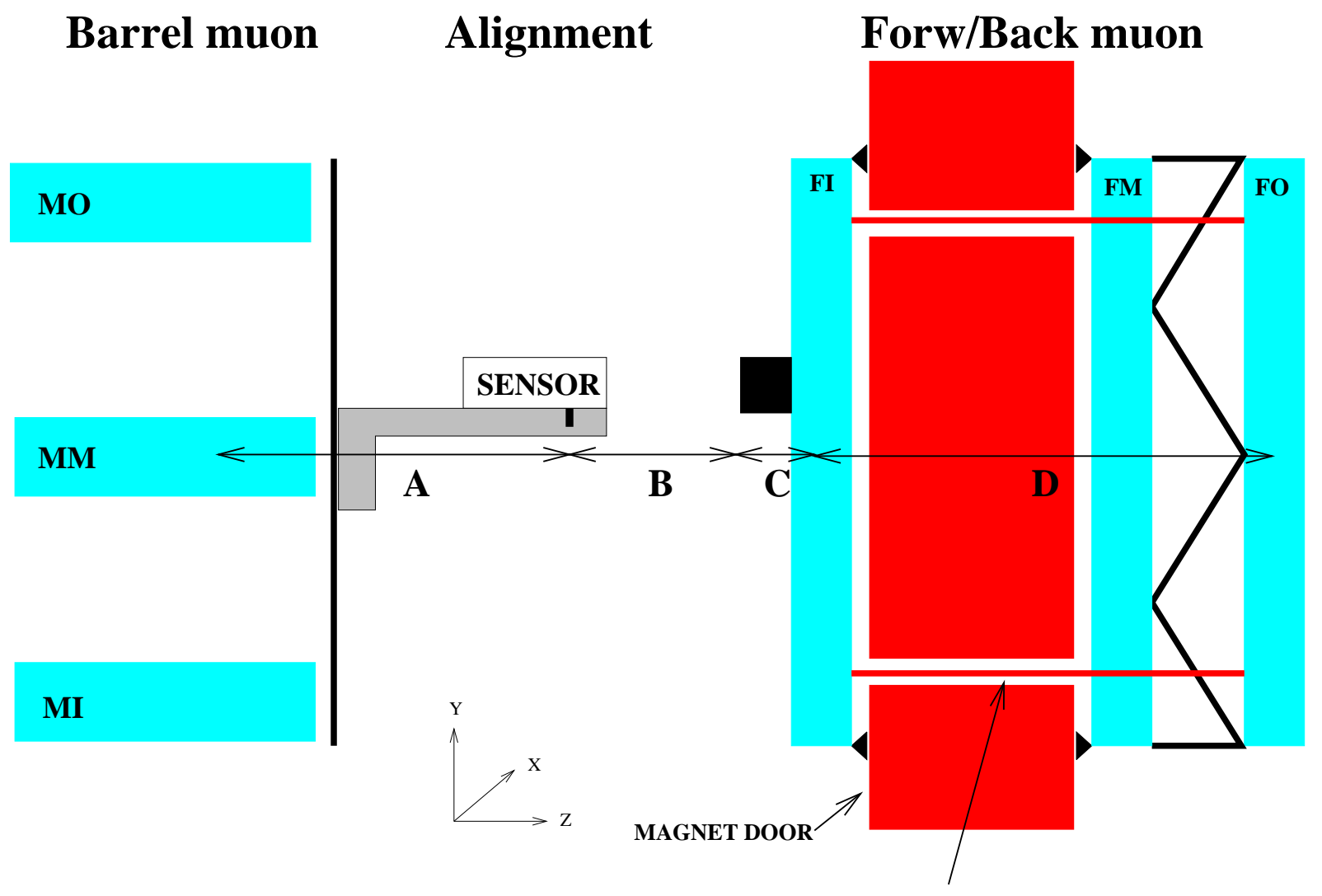

FBRASNIK SYSTEM

Figure 29: Overview of the alignment system in the F/B region, not to scale. The alignment consists of 4 steps: (A) positioning of the sensor's reference marks with respect to the barrel chamber wires, (B) a distance measurement by a sensor with respect to FI surfaces, (C) positioning of these reference surfaces with respect to the FI chamber wires and (D) the internal alignment of the three F/B layers by means of a RASNIK system. 


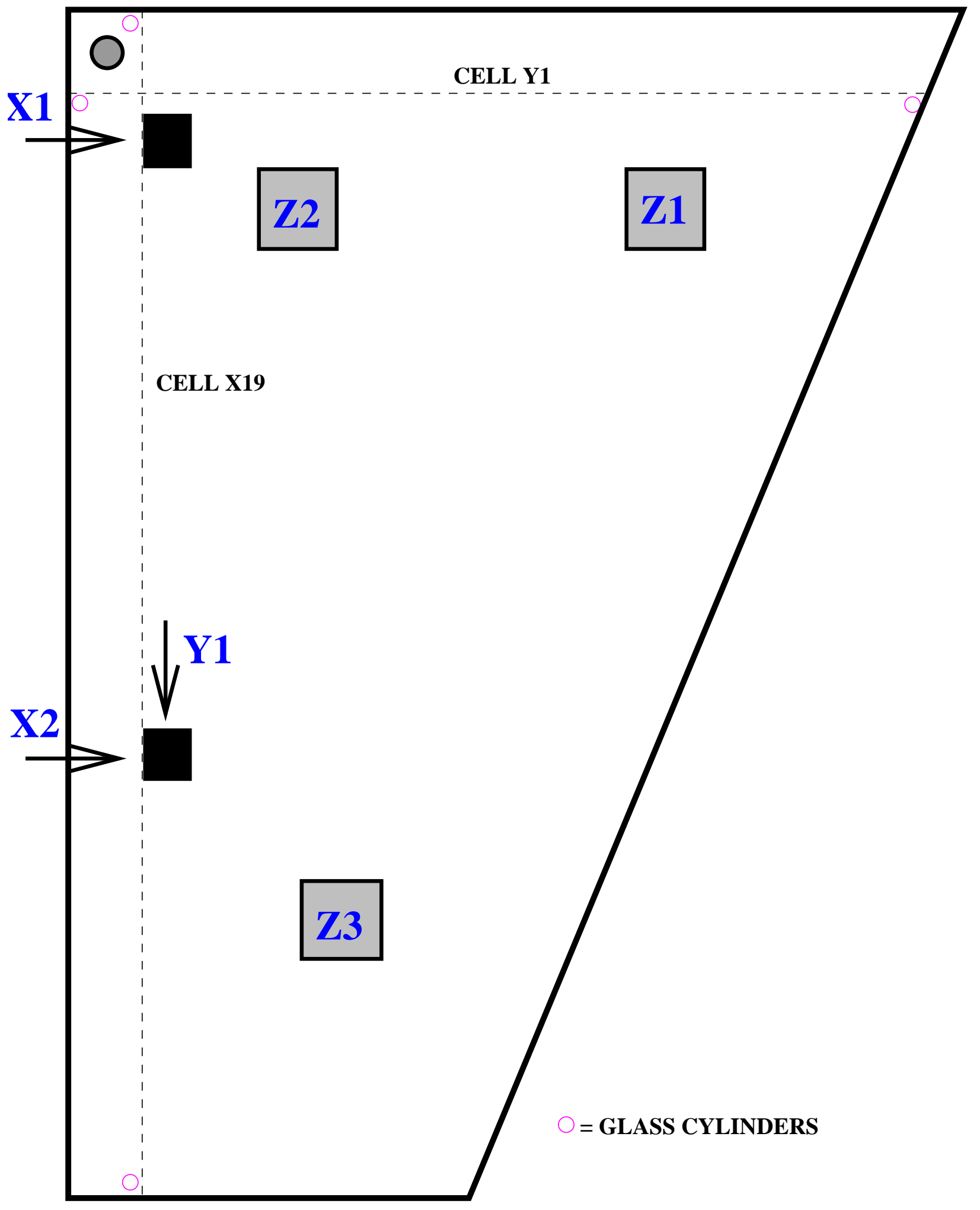

Figure 30: A front view of an FI chamber showing the position of the reference marks for $x, y$ and $z$. 


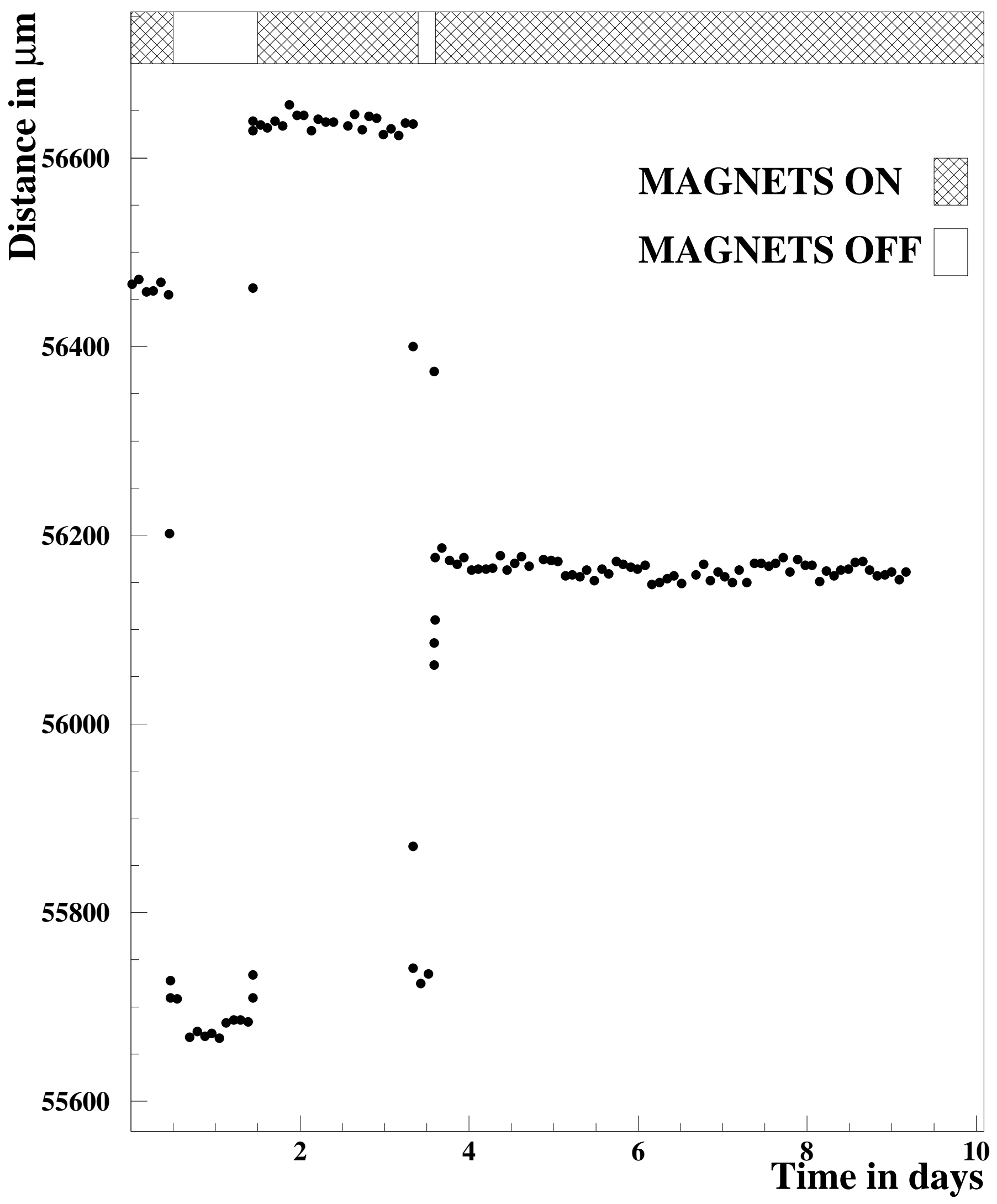

Figure 31: Distance between the muon barrel and the reference marks of a FI chamber recorded by a triangulation sensor over 10 days. The top bar indicates the status of the L3 magnets. A difference in chamber position of about $500 \mu \mathrm{m}$ can be seen for the two periods with both magnets powered. 


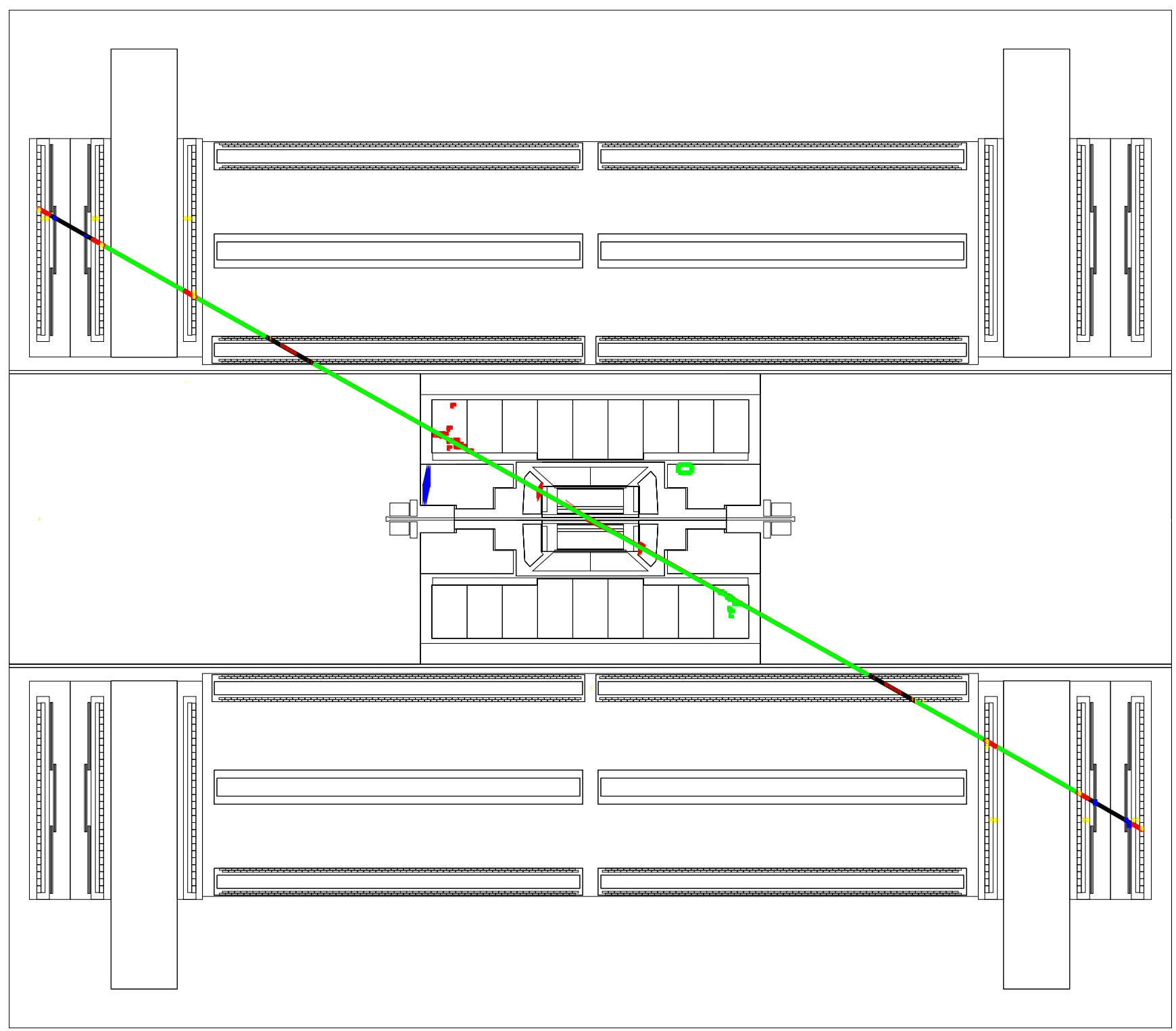

Figure 32: Side view of a dimuon event in data taken at LEP. 

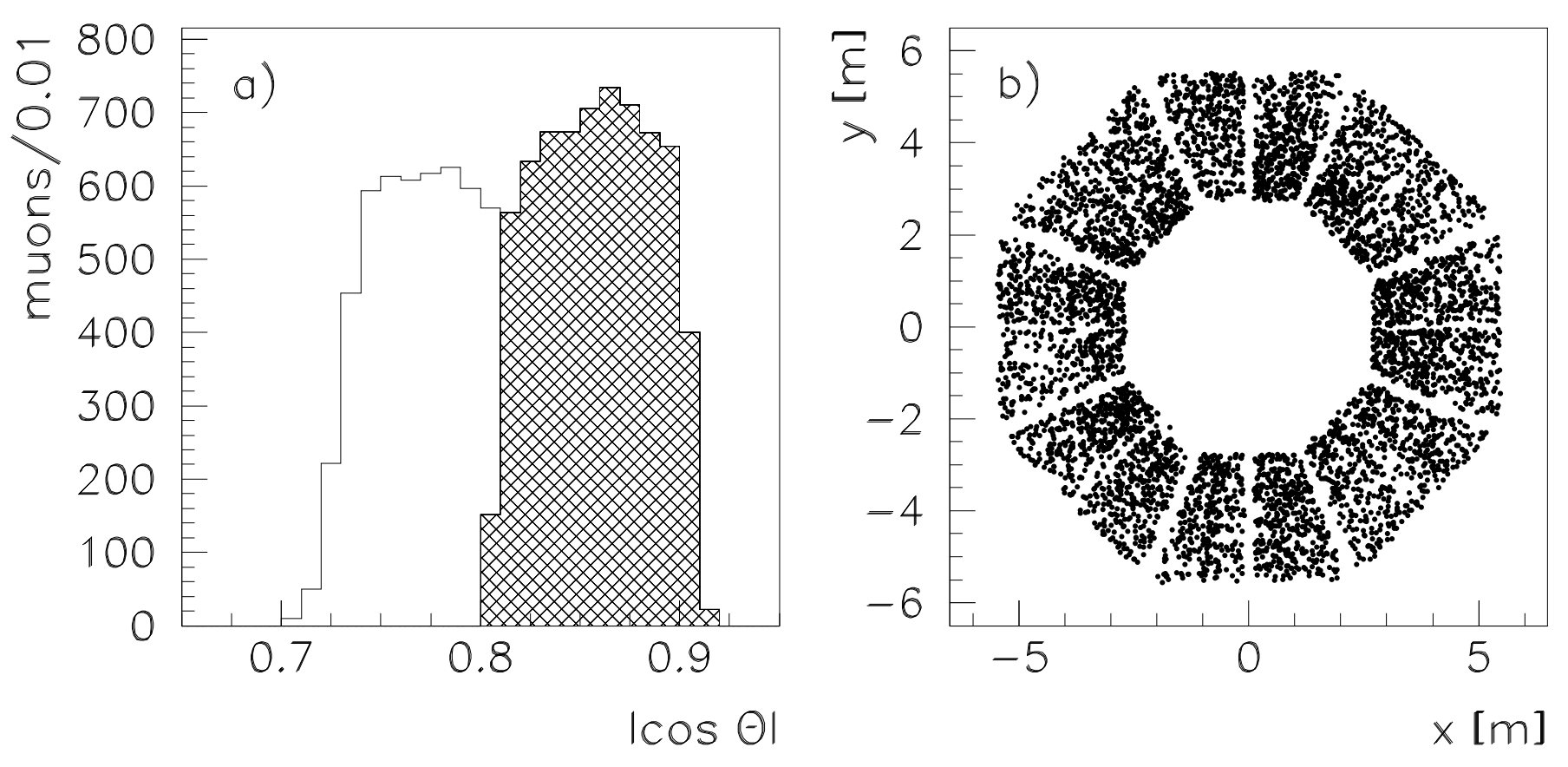

Figure 33: a): Distribution of $|\cos \Theta|$ of muons with track segments in the $\mathrm{F} / \mathrm{B}$ muon chambers. The hatched histogram shows the muons in the toroidal region. b): Impact points in the $x-y$ plane of reconstructed muon tracks in the FI chambers on one door.
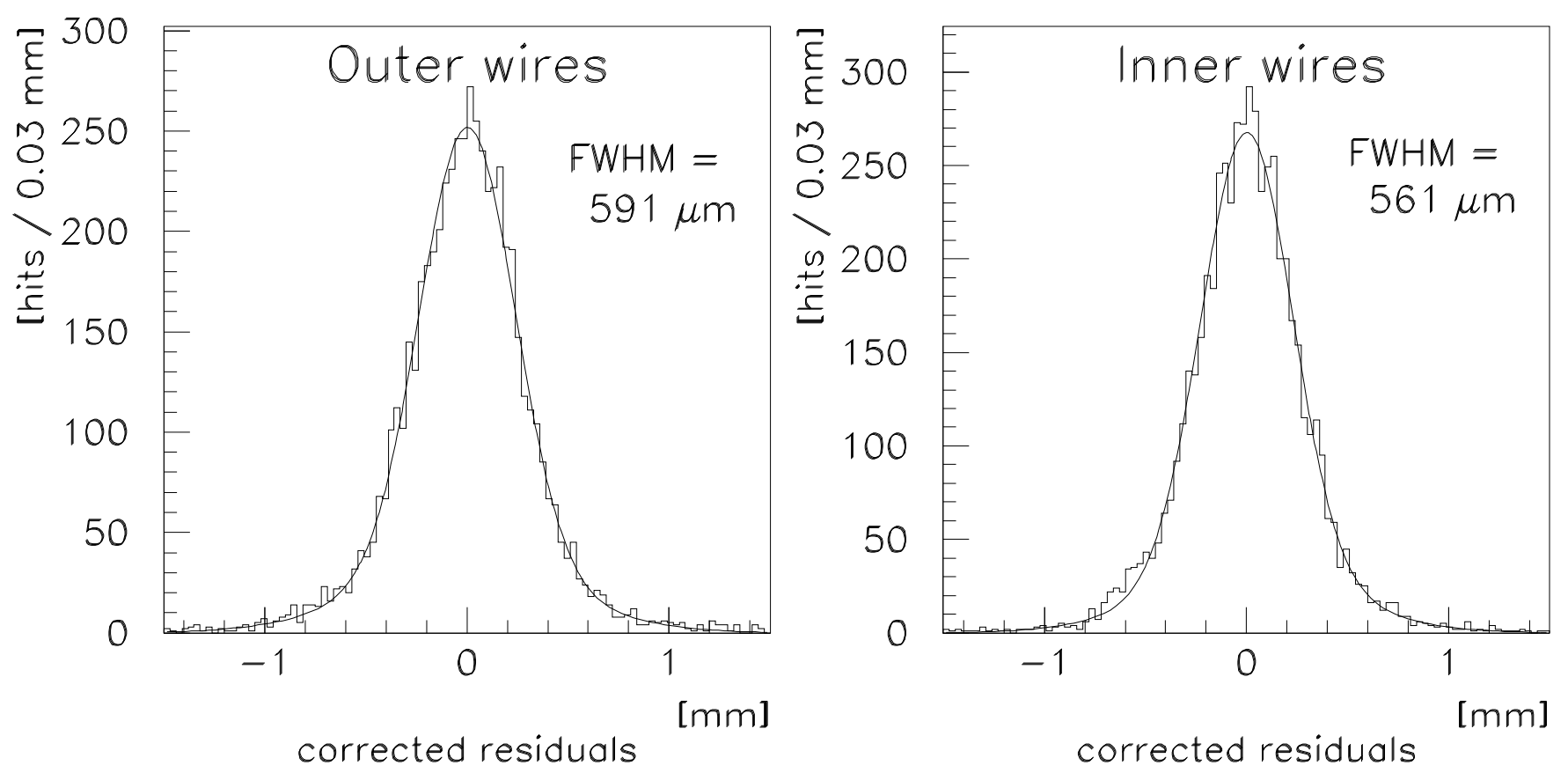

Figure 34: Corrected residuals for hits in the outer wires and inner wires for 8 point fits in FM and FO X and W layers. The Full Widths at Half Maximum (FWHM) of the distributions are shown in the plots. A detailed discussion of the resolution is given in paragraph 8.3 . 

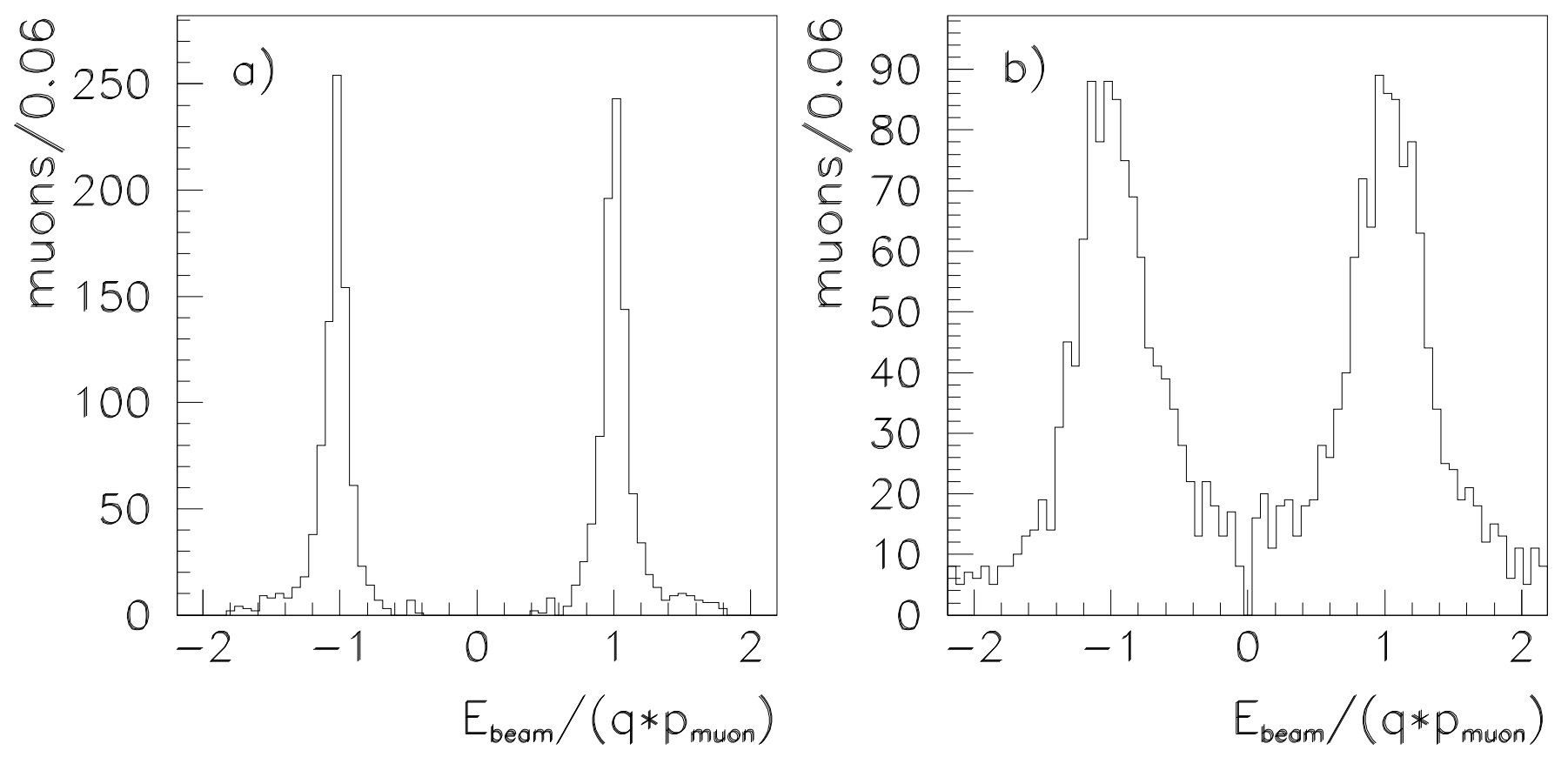

Figure 35: Momentum spread of reconstructed muons in $e^{+} e^{-} \rightarrow \mu^{+} \mu^{-}(\gamma)$ relative to the beam energy. Plotted is the quantity $E_{\text {beam }} /\left(q \cdot p_{\text {muon }}\right)$, where $E_{\text {beam }}$ is the LEP beam energy, $q$ is the reconstructed muon charge, and $p_{\text {muon }}$ the reconstructed muon momentum, in the S-region (a) and in the T-region (b). 


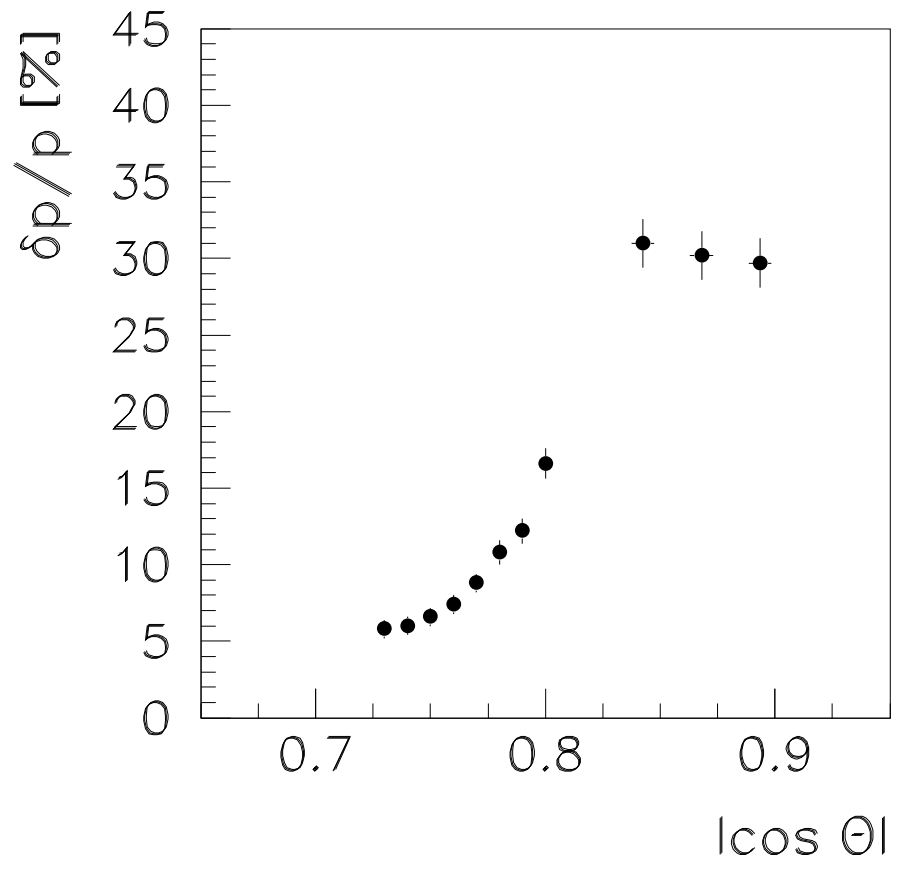

Figure 36: Muon momentum resolution obtained in the $\mathrm{F} / \mathrm{B}$ muon system as a function of the cosine of the polar angle $\Theta$ of the track, for a muon momentum of $45 \mathrm{GeV}$.

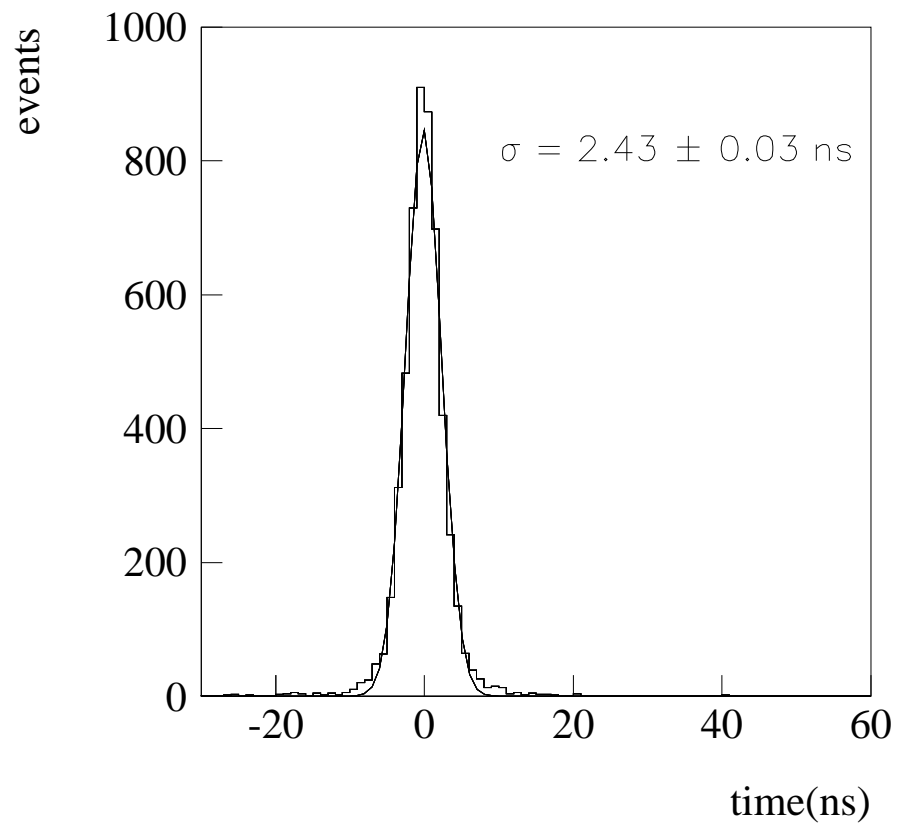

Figure 37: Time resolution 


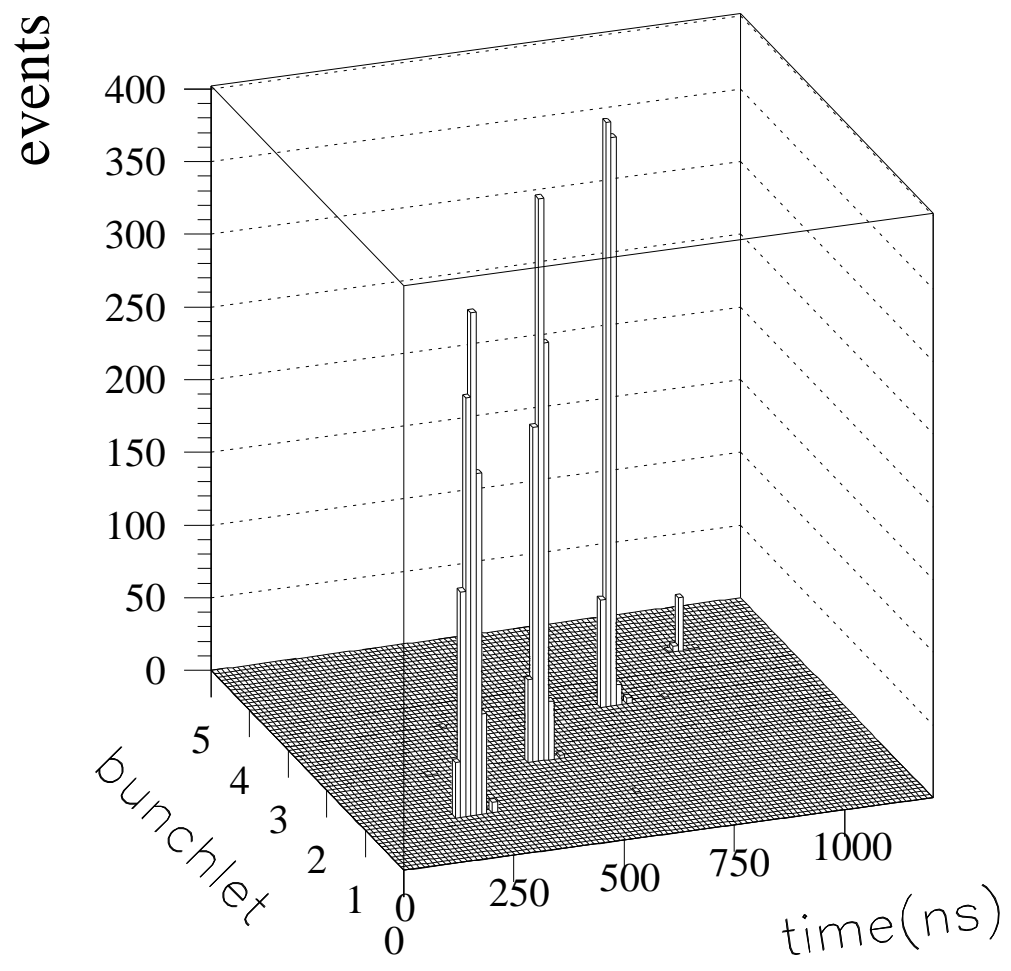

Figure 38: RPCs time distribution, without subtracting the inter-bunchlet time, versus the RPCs identified bunchlet number. 